Pacific

Journal of

Mathematics

IRREDUCIBILITY OF TENSOR SQUARES, SYMMETRIC SQUARES AND ALTERNATING SQUARES

Kay Magaard, Gunter Malle, and Pham Huu TieP

Volume $202 \quad$ No. 2

February 2002 


\title{
IRREDUCIBILITY OF TENSOR SQUARES, SYMMETRIC SQUARES AND ALTERNATING SQUARES
}

\author{
Kay Magaard, Gunter Malle, and Pham Huu TieP
}

We investigate the question when the tensor square, the alternating square, or the symmetric square of an absolutely irreducible projective representation $V$ of an almost simple group $G$ is again irreducible. The knowledge of such representations is of importance in the description of the maximal subgroups of simple classical groups of Lie type. We show that if $G$ is of Lie type in odd characteristic, either $V$ is a Weil representation of a symplectic or unitary group, or $G$ is one of a finite number of exceptions. For $G$ in even characteristic, we derive upper bounds for the dimension of $V$ which are close to the minimal possible dimension of nontrivial irreducible representations. Our results are complete in the case of complex representations. We will also answer a question of B. H. Gross about finite subgroups of complex Lie groups $\mathfrak{G}$ that act irreducibly on all fundamental representations of $\mathfrak{G}$.

\section{Introduction.}

Let $R=R\left(\ell^{f}\right)$ be a finite classical group of Lie type. Let $G<R$ be a quasi-simple subgroup acting absolutely irreducibly on the natural module of $R$, not of Lie type in characteristic $\ell$. In continuation of [18] we study those cases where $G$ has the same number of composition factors on the adjoint module for $R$ as $R$ itself. These embeddings are of importance in the determination of maximal subgroups of the finite classical groups of Lie type.

Let $V$ be the natural module for $R$. We will write $\widetilde{\Lambda}^{2}(V), \widetilde{\Sigma}^{2}(V)$ respectively $\widetilde{A}(V)$ for the largest irreducible $R$-sub-quotient of $\Lambda^{2}(V), \operatorname{Sym}^{2}(V)$, $V \otimes V^{*}$. In Table 1.1 we recall the dimension of $X(V)$ for certain choices $(R, X)$ with $X \in\left\{\widetilde{\Lambda}^{2}, \widetilde{\Sigma}^{2}, \widetilde{A}\right\}$.

In this paper we study quasi-simple subgroups $G$ of classical groups $R$ which act irreducibly on $V$ as well as on $X(V)$ with $X$ as in Table 1.1. It is known that the following families of examples do occur:

(1) $V$ is the heart of the natural permutation module of $G=\mathfrak{A}_{n}$ (see [18]),

(2) $V$ is a Weil module of $G=\operatorname{Sp}_{2 n}(q), q \in\{3,5,9\}$, (see $[\mathbf{1 9}, \mathbf{2 0}]$ ), 
Table 1.1. Choices for $R$ and $X$.

\begin{tabular}{|l|cll|}
\hline$R$ & $X$ & $\operatorname{dim}(X(V))$ & condition \\
\hline $\mathrm{SL}_{m}$ & $\widetilde{A}$ & $m^{2}-1$ & $\ell \not \nmid m$ \\
& $\widetilde{A}$ & $m^{2}-2$ & $\ell \mid m$ \\
$\mathrm{Sp}_{m}$ & $\widetilde{\Sigma}^{2}$ & $\frac{1}{2} m(m+1)$ & $\ell$ odd \\
& $\widetilde{\Sigma}^{2}$ & $\frac{1}{2} m(m-1)-1$ & $\ell=2, m \equiv 2(\bmod 4)$ \\
& $\widetilde{\Sigma}^{2}$ & $\frac{1}{2} m(m-1)-2$ & $\ell=2, m \equiv 0(\bmod 4)$ \\
$\mathrm{SO}_{m}$ & $\widetilde{\Lambda}^{2}$ & $\frac{1}{2} m(m-1)$ & $\ell$ odd \\
& $\widetilde{\Lambda}^{2}$ & $\frac{1}{2} m(m-1)-1$ & $\ell=2, m \equiv 2(\bmod 4)$ \\
& $\widetilde{\Lambda}^{2}$ & $\frac{1}{2} m(m-1)-2$ & $\ell=2, m \equiv 0(\bmod 4)$ \\
& $\widetilde{\Sigma}^{2}$ & $\frac{1}{2} m(m+1)-1$ & $\ell \not \nmid m$ \\
& $\widetilde{\Sigma}^{2}$ & $\frac{1}{2} m(m+1)-2$ & $\ell \mid m, \ell$ odd \\
\hline
\end{tabular}

(3) $V$ is a Weil module of $G=\mathrm{SU}_{n}(q), q \in\{2,3\}$ (see $[\mathbf{2 0}, \mathbf{1 4}]$ ),

(4) $V$ is a module of dimension $\left(2^{n}-1\right)\left(2^{n-1}-1\right) / 3,\left(2^{n}+1\right)\left(2^{n-1}+1\right) / 3$ or $\left(2^{2 n}-1\right) / 3$ for $G=\operatorname{Sp}_{2 n}(2)$ and $X=\widetilde{\Lambda}^{2}$ (see Prop. 7.4).

We expect that the above are the only infinite series of examples. Our main result is somewhat weaker; in order to formulate it denote by $l(G)$ the (known lower bounds for the) minimal dimensions of nontrivial representations (Landázuri-Seitz-Zalesskii bound):

Theorem 1.2. Let $G$ be quasi-simple and $V$ a nontrivial absolutely irreducible representation in characteristic $\ell \geq 0$ (which is different from the defining characteristic if $G$ is of Lie type). Let $X=\widetilde{A}$ if $V$ is not self-dual, and $X=\widetilde{\Sigma}^{2}$ or $\widetilde{\Lambda}^{2}$ otherwise. Then one of

(i) $X(V)$ is reducible, or

(ii) $(G, V)$ are as in (1)-(4) above, or

(iii) $G=G(q)$ is classical, $q \in\{2,4,8\}$ and $\operatorname{dim}(V)$ is at most $c \cdot l(G)^{2}$, or

(iv) $G=G(q)$ is exceptional, $q$ is even and $\operatorname{dim}(V)$ is at most $4 l(G)$, or

(v) $G$ is on a known finite list of groups.

More precise formulations can be found in Theorem 3.1 and the Propositions in Section 5.

Clearly this result is true for the finitely many sporadic groups, see also Section 6. Complete results for alternating and special linear groups were obtained by the first two authors [18] (see also the references given there). The case of complex representations is completely solved in Theorem 7.14. Observe that the classification of complex modules $V$ with irreducible $\widetilde{\Sigma}^{2}(V)$ 
has interesting applications in the theory of integral Euclidean lattices, cf. $[14]$.

Our analysis of the remaining groups of Lie type splits into two cases, the second of which only occurs for groups defined over fields of characteristic 2 . The first case leads to the examples in (2) and (3) above. In the second case we either show that $X(V)$ is reducible or at least derive upper bounds for $\operatorname{dim}(V)$ which are very close to $l(G)$, the worst case being that of groups in characteristic 2 over fields of characteristic 3.

Also we improve the Landázuri-Seitz-Zalesskii bounds for the twisted exceptional groups ${ }^{3} D_{4}(q)$ and ${ }^{2} E_{6}(q)$, which might be of independent interest (see Section 4). To our knowledge, this only leaves the groups of types ${ }^{2} F_{4}$ and $F_{4}$ for which no sharp lower bound for the dimension of nontrivial representations in cross characteristic has been proved.

The finite irreducible complex reflection groups $G$ are known to have the property that all exterior powers of their reflection representations remain irreducible. This can be rephrased by saying that $G$ is a subgroup of $\mathfrak{G}=$ $\mathrm{SL}_{n}(\mathbb{C})$ acting irreducibly in all fundamental representations of $\mathfrak{G}$. In the final section of our paper we determine all finite subgroups of complex simple simply-connected Lie groups with this property, thus answering a question asked by B.H. Gross:

Theorem 1.3. Let $G$ be a finite subgroup of a complex simple simply-connected Lie group $\mathfrak{G}$ which is irreducible in all fundamental representations of $\mathfrak{G}$. Assume that the dimension d of the natural module $V$ for $\mathfrak{G}$ is at least 5 and $\mathfrak{G} \neq \operatorname{Spin}_{5}(\mathbb{C}) \cong \operatorname{Sp}_{4}(\mathbb{C}), \mathfrak{G} \neq \operatorname{Spin}_{6}(\mathbb{C}) \cong \mathrm{SL}_{4}(\mathbb{C})$. Then up to a finite subgroup of the center $Z(\mathfrak{G})$ one of the following holds, where $\bar{G}$ denotes the image of $G$ in its action on the natural module for $\mathfrak{G}$ :

(i) $G=k^{m} \cdot H$ is monomial on the natural module with $k \geq 2, d-1 \leq$ $m \leq d, \mathfrak{G}=\mathrm{SL}_{d}(\mathbb{C})$, and $\mathfrak{A}_{d} \leq H \leq \mathfrak{S}_{d}$ or $H$ is as in Table 7.18.

(ii) $\bar{G}=2^{m} . H$ is monomial on the natural module with $d-1 \leq m \leq d$, $\mathfrak{G}=\operatorname{Spin}_{d}(\mathbb{C})$ and $\mathfrak{A}_{d} \leq H \leq \mathfrak{S}_{d}$, or $H$ is as in Table 7.18.

(iii) $G=2^{3} \cdot \mathrm{SL}_{3}(2)$ and $\mathfrak{G}=G_{2}(\mathbb{C})$.

(iv) $G \leq 5_{+}^{1+2}: \mathrm{SL}_{2}(5)$ and $\mathfrak{G}=\mathrm{SL}_{5}(\mathbb{C})$.

(v) $\bar{G} \leq 2_{+}^{1+6} \cdot \mathfrak{S}_{8}$ and $\mathfrak{G}=\operatorname{Spin}_{8}(\mathbb{C})$.

(vi) $G$ is almost quasi-simple and $(\mathfrak{G}, G)$ are as in Table 7.22.

In particular, except for finitely many cases, $G$ contains the derived group of an irreducible complex reflection group.

\section{Generalities.}

Let $G$ be a finite group and $\mathbb{F}$ an algebraically closed field of characteristic $\ell$. 
Let $V$ be any irreducible $\mathbb{F} G$-module. We say that $V$ is of type + if it carries a nondegenerate $G$-invariant quadratic form, and that $V$ is of type if it carries a bilinear alternating but no quadratic form. We would like to define some modules arising from $V$. If $V$ is not of type + (resp. not of type -), then $\widetilde{\Sigma}^{2}(V)$ (resp. $\left.\widetilde{\Lambda}^{2}(V)\right)$ denotes $\operatorname{Sym}^{2}(V)\left(\right.$ resp. $\left.\Lambda^{2}(V)\right)$. Next, let $Y$ be $V \otimes V^{*}$ if $V$ is not self-dual, $\operatorname{Sym}^{2}(V)$ if $V$ is of type,$+ \Lambda^{2}(V)$ if $V$ is of type - . Then $Y$ is self-dual and $\operatorname{dim} \operatorname{Hom}_{G}\left(Y, 1_{G}\right)=\operatorname{dim}_{\operatorname{Hom}_{G}}\left(1_{G}, Y\right)=1$. Let $T$ be the (unique) submodule of $Y$ such that $Y / T \simeq 1_{G}$, and let $I$ be the unique trivial submodule of $Y$. Then we will denote $T /(T \cap I)$ by $\widetilde{A}(V)$, resp. $\widetilde{\Sigma}^{2}(V), \widetilde{\Lambda}^{2}(V)$. If $V$ is a composition factor of an $\mathbb{F} G$-module $U$, we will write $V \hookrightarrow U$.

According to Table 1.1, $\widetilde{A}(V), \widetilde{\Sigma}^{2}(V), \widetilde{\Lambda}^{2}(V)$ respectively is irreducible for the ambient classical group.

Lemma 2.1. Let $U$ be a uniserial $\mathbb{F} G$-module with $\operatorname{soc}(U)=I$ being the trivial module and $U / I=V$ being irreducible, and $V \neq I$. Let $D$ be a subgroup of $G, L$ a one-dimensional $\mathbb{F} D$-module, and let $W=L \uparrow G$. Suppose that $\operatorname{Hom}_{G}(U, W)$ has dimension $t \geq 1$. Then $V$ is a composition factor of $W$ of multiplicity at least $t$.

Proof. Pick a nonzero map $f \in \operatorname{Hom}_{G}(U, W)$. Since $U$ is uniserial, $V \hookrightarrow$ $f(U)$, hence $V \hookrightarrow W$ and we get the statement for the case $t=1$.

Next suppose that $t \geq 2$ and fix a basis $\left(f_{1}, \ldots, f_{t}\right)$ of $\operatorname{Hom}_{G}(U, W)$. Let $v$ be a generator of $I$. If $f_{i}(v)=0$ for all $i$, then $f_{i} \in \operatorname{Hom}_{G}(V, W)$ for all $i$, whence $\operatorname{dim} \operatorname{Hom}_{G}(V, W)=t$ and the multiplicity of $V$ in $W$ is at least $t$, as stated. Suppose $f_{1}(v) \neq 0$. Observe that $\operatorname{dim} \operatorname{Hom}_{G}(I, W)=$ $\operatorname{dim} \operatorname{Hom}_{D}\left(1_{D}, L\right) \leq 1$, since $\operatorname{dim} L=1$. Hence $f_{i}(v)=\lambda_{i} f_{1}(v)$ for some $\lambda_{i} \in \mathbb{F}$. Replacing $f_{i}$ by $f_{i}-\lambda_{i} f_{1}$, we may assume that $f_{i}(v)=0$ for all $i \geq 2$. Let $V^{\prime}=\sum_{i=2}^{t} f_{i}(U)$. Then $V^{\prime}$ is a sum of some copies of $V$. But $f_{2}, \ldots, f_{t}$ are linearly independent elements of $\operatorname{Hom}_{G}\left(V, V^{\prime}\right)$. Therefore $V^{\prime}$ is a direct sum of $t-1$ copies of $V$. Finally, let $W^{\prime}=f_{1}(I)+V^{\prime}$. Then $W^{\prime} \simeq I \oplus(t-1) V$. Since $U$ is uniserial, $f_{1}(U) \nsubseteq W^{\prime}$. Thus

$$
V \simeq f_{1}(U) / f_{1}(I)=f_{1}(U) /\left(f_{1}(U) \cap W^{\prime}\right) \simeq\left(f_{1}(U)+W^{\prime}\right) / W^{\prime} \subseteq W / W^{\prime},
$$

whence $W / W^{\prime}$ has $V$ as a composition factor, and so the multiplicity of $V$ in $W$ is at least $t$.

Corollary 2.2. Let $U$ be a uniserial $\mathbb{F} G$-module with $\operatorname{soc}(U)=V$ being irreducible, $U / V \simeq I$ being the trivial module, and $V \neq I$. Let $D$ be a subgroup of $G, L$ a one-dimensional $\mathbb{F} D$-module, and let $W=L \uparrow G$. Suppose that $\operatorname{Hom}_{G}(W, U)$ has dimension $t \geq 1$. Then $V$ is a composition factor of $W$ of multiplicity at least $t$.

Proof. Dualize Lemma 2.1. 
A key ingredient of our arguments is the following proposition, in which $X(V)$ means either $\widetilde{\Sigma}^{2}(V)$ or $\widetilde{\Lambda}^{2}(V)$ if $V$ is self-dual, and $\widetilde{A}(V)$ if $V$ is not self-dual.

Proposition 2.3. Let $V$ be an irreducible $\mathbb{F} G$-module such that $X(V)$ is irreducible. Let $Z$ be an abelian $\ell^{\prime}$-subgroup of $G$ and denote $C=C_{G}(Z)$, $N=N_{G}(Z)$. Suppose that $\left.V\right|_{Z}$ affords at least $t \geq 2$ linear characters $\alpha_{i}$, $1 \leq i \leq t$, no two of which are dual to each other.

(i) If $(\operatorname{type}(V), X) \in\left\{(\circ, \widetilde{A}),\left(+, \widetilde{\Sigma}^{2}\right),\left(-, \widetilde{\Lambda}^{2}\right)\right\}$, then $X(V)$ is a composition factor of $\left(1_{C}\right) \uparrow^{G}$ of multiplicity at least $t-1$. If in addition at least one $\alpha_{i}$ is $N$-invariant, then $X(V) \hookrightarrow\left(1_{N}\right) \uparrow^{G}$ and so $\operatorname{dim} X(V) \leq(G$ : $N)$. If $N / C$ is abelian then we also have $\operatorname{dim} X(V) \leq(G: N)$.

(ii) If (type $(V), X) \in\left\{\left(-, \widetilde{\Sigma}^{2}\right),\left(+, \widetilde{\Lambda}^{2}\right)\right\}$ and $|Z|$ is odd, then $X(V)$ is a composition factor of $\left(1_{C}\right) \uparrow G$ of multiplicity at least $t-1$. If in addition $N / C$ is abelian, then $\operatorname{dim} X(V) \leq(G: N)$.

Proof. 1) Observe that the $\mathbb{F} Z$-module $V$ is semisimple. In Cases (i) and (ii) we put $D=C$ and write $\left.V\right|_{Z}=A_{1} \oplus \ldots \oplus A_{s}$, where $s=t, A_{i}$, $1 \leq i \leq s-1$, can afford only the $Z$-characters $\alpha_{i}$ and $\bar{\alpha}_{i}$, and $A_{s}$ affords all the rest of $Z$-characters. In Case (i), if $\alpha_{i}$ is $N$-invariant, we can also put $s=2$ and $D=N$. Assuming for definiteness that $\alpha_{1}$ is $N$-stable, we write $\left.V\right|_{Z}=A_{1} \oplus A_{2}$, where $A_{1}$ can afford only $\alpha_{1}$ and $\bar{\alpha}_{1}$, and $A_{2}$ affords all the rest of $Z$-characters. Clearly, each $A_{i}$ is $D$-stable, hence we can view $A_{i}$ as an $\mathbb{F} D$-module. The construction of $A_{i}$ ensures that

$$
\operatorname{Hom}_{Z}\left(A_{i}, A_{j}\right)=\operatorname{Hom}_{Z}\left(A_{i}, A_{j}^{*}\right)=0
$$

whenever $i \neq j$. In particular, $\operatorname{Hom}_{D}\left(A_{i}, A_{j}\right)=\operatorname{Hom}_{D}\left(A_{i}, A_{j}^{*}\right)=0$ if $i \neq j$. We will let $W=\left(1_{D}\right) \uparrow^{G}$.

2) Here we consider the subcase $X(V)=\widetilde{A}(V)$ of (i).

2a) Take $D=C, s=t$. Then

$$
\left.\left(V \otimes V^{*}\right)\right|_{D}=\sum_{i=1}^{s} A_{i} \otimes A_{i}^{*} \oplus \sum_{1 \leq i \neq j \leq s} A_{i} \otimes A_{j}^{*} .
$$

As mentioned above, $\operatorname{Hom}_{D}\left(1_{D}, A_{i} \otimes A_{j}^{*}\right)=0$ if $i \neq j$. Hence the trivial $\mathbb{F} G$ module $I$ inside $V \otimes V^{*}$ has to be contained (as a subspace) in $\sum_{i}\left(A_{i} \otimes A_{i}^{*}\right)$. Let $J$ be the sum of the $s$ (nonzero) $D$-fixed point subspaces inside $A_{i} \otimes A_{i}^{*}$, $1 \leq i \leq s$. Then $I \subset J$ and

$$
\operatorname{dim} \operatorname{Hom}_{D}\left(1_{D}, J / I\right) \geq s-1,
$$

whence

$$
s-1 \leq \operatorname{dim} \operatorname{Hom}_{D}\left(1_{D}, Y(V) / I\right)=\operatorname{dim} \operatorname{Hom}_{G}(W, Y(V) / I),
$$


(recall $Y(V)=V \otimes V^{*}$ in this case). If $T \cap I=0$, then $\widetilde{A}(V) \simeq Y(V) / I$, and $\widetilde{A}(V)$ is irreducible by assumption, so $\widetilde{A}(V)$ has multiplicity at least $s-1$ in $W$. Suppose $T \supseteq I$. Then $Y(V) / I$ is a uniserial $\mathbb{F} G$-module with socle equal $\widetilde{A}(V)$. By Corollary 2.2, the multiplicity of $\widetilde{A}(V)$ in $W$ is again at least $s-1$ as stated.

2b) The same argument yields the statement for the subcase $X(V)=$ $\widetilde{A}(V)$ and $\alpha_{i}$ is $N$-invariant: We just need to put $D=N$ and $s=2$.

2c) Now suppose that $N / C$ is abelian. According to $(2.5), \operatorname{Hom}_{C}\left(1_{C}\right.$, $Y(V) / I) \neq 0$. Thus the subspace $F$ of $C$-fixed points in $Y(V) / I$ is nonzero, and $F$ is acted on by $N$. But $N / C$ is abelian, hence the $N / C$-module $F$ contains a one-dimensional $\mathbb{F} N$-module $L$. In this case $0 \neq \operatorname{Hom}_{N}(L, Y(V) / I) \simeq$ $\operatorname{Hom}_{G}\left(L \uparrow^{G}, Y(V) / I\right)$. Arguing as in 2a) and using Corollary 2.2 if $T \supseteq I$, we get $\widetilde{A}(V) \hookrightarrow L^{\uparrow}$; in particular, $\operatorname{dim} \widetilde{A}(V) \leq(G: N)$.

3) It remains to consider the subcases $X(V)=\widetilde{\Sigma}^{2}(V)$ and $\widetilde{\Lambda}^{2}(V)$. Take $D=C, s=t$. Then $V$ supports a nondegenerate $G$-invariant bilinear form $b$. We claim that $\left.b\right|_{A_{i}}$ is also nondegenerate. For, let $A_{i}^{\perp}$ be the orthogonal complement to $A_{i}$ in $V$ (with respect to $b$ ) and $B=\sum_{j \neq i} A_{j}$. Then $B /\left(B \cap A_{i}^{\perp}\right) \simeq\left(B+A_{i}^{\perp}\right) / A_{i}^{\perp} \subseteq V / A_{i}^{\perp} \simeq A_{i}^{*}$. But due to (2.4), the $Z$-modules $B$ and $A_{i}^{*}$ have no common composition factors. Hence $B \subseteq A_{i}^{\perp}$. Comparing the dimension we get $B=A_{i}^{\perp}$ and so $A_{i} \cap A_{i}^{\perp}=0$, as stated. Now for $Y(V)=\operatorname{Sym}^{2}(V)$ or $\Lambda^{2}(V)$ we have

$$
\left.Y(V)\right|_{D}=\sum_{i=1}^{s} Y\left(A_{i}\right) \oplus \sum_{1 \leq i<j \leq s} A_{i} \otimes A_{j} .
$$

4a) In Case (i), let $J$ be the sum of the $D$-fixed point subspaces inside $Y\left(A_{i}\right), 1 \leq i \leq s$; all of them are nonzero because of the nondegeneracy of $\left.b\right|_{A_{i}}$. Then $\operatorname{dim} J \geq s$. By (2.4) above, $\operatorname{Hom}_{D}\left(1_{D}, A_{i} \otimes A_{j}\right)=0$ if $i \neq j$. Hence the trivial $\mathbb{F} G$-module $I$ inside $Y(V)$ has to be contained (as a subspace) in $J$. We again have

$$
\operatorname{dim} \operatorname{Hom}_{D}\left(1_{D}, J / I\right) \geq s-1
$$

and so $\operatorname{dim} \operatorname{Hom}_{G}(W, Y(V) / I) \geq s-1$. At this point we can repeat the arguments of 2a) to show that the multiplicity of $X(V)$ in $W$ is at least $s-1$.

If in addition $\alpha_{1}$ is $N$-invariant, then we can use the same argument, with changing $D$ to $N$ and $s$ to 2 .

Suppose $N / C$ is abelian. According to (2.6), $\operatorname{Hom}_{C}\left(1_{C}, Y(V) / I\right) \neq 0$. At this point we can repeat the argument of $2 \mathrm{c}$ ).

4b) Finally, we consider Case (ii). Note that, if $\ell=2$, we may argue as above. Thus we may now assume that $Y(V)=X(V)$ is irreducible. By $3)$, the restriction to $A_{i}$ of the nondegenerate $G$-invariant bilinear form $b$ is nondegenerate. But unlike Case $4 \mathrm{a})$, now we cannot conclude that $Y\left(A_{i}\right)$ 
has nonzero $D$-fixed points for every $i$ (the form $b$ is not of the right type!). We may assume that $\alpha_{i} \neq 1_{Z}$ for $i \geq 2$. We claim that for each $i \geq 2, Y\left(A_{i}\right)$ has nonzero $D$-fixed points. For, since $V$ is self-dual, $\left.A_{i}\right|_{Z}=B_{i} \oplus B_{i}^{\prime}$, where $B_{i}$ affords only $\alpha_{i}$ (with some multiplicity) and $B_{i}^{\prime}$ affords only $\bar{\alpha}_{i}$. Since $|Z|$ is odd, $\alpha_{i} \neq \bar{\alpha}_{i}$. Clearly, $B_{i}$ and $B_{i}^{\prime}$ are $D$-stable. Again by the self-duality of $V, B_{i}^{\prime} \simeq B_{i}^{*}$ as $D$-modules. Now $B_{i} \otimes B_{i}^{*}$ is a submodule of $Y\left(A_{i}\right)$, and $B_{i} \otimes B_{i}^{*}$ has nonzero $D$-fixed points, hence the claim follows.

Let $J$ be the sum of the $D$-fixed point subspaces inside $Y\left(A_{i}\right), 1 \leq i \leq s$. Then $\operatorname{dim} J \geq s-1$. Therefore

$$
\operatorname{dim} \operatorname{Hom}_{D}\left(1_{D}, J\right) \geq s-1
$$

and so $\operatorname{dim} \operatorname{Hom}_{G}(W, Y(V)) \geq s-1$. Since $X(V)=Y(V)$ in (ii), we conclude that the multiplicity of $X(V)$ in $W$ is at least $s-1$.

Now suppose in addition that $N / C$ is abelian. According to (2.7) we have that $\operatorname{Hom}_{C}\left(1_{C}, X(V)\right) \neq 0$. It remains to repeat the argument of $\left.2 \mathrm{c}\right)$.

In what follows, we will apply Proposition 2.3 in the following set-up: $G$ is a finite group of Lie type, defined over a field $\mathbb{F}_{q}$ in characteristic $p$ and of universal type, and $Z$ is a long-root subgroup $\left\{x_{\alpha}(t) \mid t \in \mathbb{F}_{q}\right\}, \alpha$ a long root. A visual description of $Z$ is given for instance in [19]. To apply Proposition 2.3 efficiently, one therefore needs to know the spectrum $\operatorname{Spec}(Z, V)$ of abelian subgroups $Z$ in any irreducible representation $V$, that is, the set of (distinct) linear characters of $Z$ occurring in $V$. If $Z=\langle g\rangle$, we denote $\operatorname{Spec}(Z, V)$ by $\operatorname{Spec}(g, V)$. The following theorem is the main result of $[\mathbf{2 7}]$ :

Theorem $2.8([\mathbf{2 7}])$. Let $G$ be a finite group of Lie type, defined in characteristic $p$ and of universal type, and $g \in G$ a non-central element of order $p$. Suppose $G$ has an irreducible representation $V$ over an algebraically closed field $\mathbb{F}$ of characteristic $\ell \neq p$ such that $1<|\operatorname{Spec}(g, V)|<p$. Then $p>2$ and one of the following holds.

(i) $G=\mathrm{SU}_{3}(p)$ or $\mathrm{Sp}_{2 n}(p)$, and $g$ is a transvection.

(ii) $G=\mathrm{SL}_{2}\left(p^{2}\right)$ or $\mathrm{Sp}_{4}(p)$.

Moreover, if $1 \notin \operatorname{Spec}(g, V)$ and $G=[G, G]$ then $p>2$ and

$$
G \in\left\{\mathrm{SL}_{2}(p), \mathrm{SL}_{2}\left(p^{2}\right), \mathrm{SU}_{3}(p), \mathrm{Sp}_{4}(p)\right\} .
$$

The group $Z$ is elementary abelian of order $q$ and may be identified with the additive group $\left\{t \mid t \in \mathbb{F}_{q}\right\}$. Fix a $p^{\text {th }}$ primitive root $\epsilon$ of unity in $\mathbb{C}$. Then any irreducible Brauer character of $Z$ in characteristic $\ell \neq p$ is of the form

$$
\lambda_{c}: t \mapsto \epsilon^{\operatorname{tr}_{\mathbb{F}_{q} / \mathbb{F} p}(t c)}
$$

for some $c \in \mathbb{F}_{q}$. Let $\Omega^{+}$, resp. $\Omega^{-}$, be the set of all $\lambda_{c}$, where $c$ is any square, resp. non-square, in $\mathbb{F}_{q}^{\times}$.

We will need the following supplement to Theorem 2.8: 
Lemma 2.9. Let $G$ be a universal-type quasi-simple finite group of Lie type defined over $\mathbb{F}_{q}, q=p^{f}$, and $Z$ a long-root subgroup as above. Let $\mathbb{F}$ be an algebraically closed field of characteristic $\ell \neq p$ and $V$ a nontrivial irreducible $\mathbb{F} G$-module. Suppose $\operatorname{Spec}(Z, V) \neq \operatorname{IBr}_{\ell}(Z)$. Then one of the following holds.

(i) $q>p$, and $\operatorname{Spec}(Z, V)=\Omega^{+} \cup \Omega^{-}$.

(ii) $G=\mathrm{SU}_{3}(p), p>2$, and $\operatorname{Spec}(Z, V)=\Omega^{+} \cup \Omega^{-}$.

(iii) $G=\operatorname{Sp}_{2 n}(q), n \geq 3, p>2$, and $\operatorname{Spec}(Z, V)=\Omega^{+} \cup\left\{1_{Z}\right\}$, or $\Omega^{-} \cup\left\{1_{Z}\right\}$.

(iv) $G=\operatorname{Sp}_{4}(q), p>2$, and $\operatorname{Spec}(Z, V)=\Omega^{+} \cup\left\{1_{Z}\right\}, \Omega^{-} \cup\left\{1_{Z}\right\}$, or $\Omega^{+} \cup \Omega^{-}$.

(v) $G=\mathrm{SL}_{2}(q), p>2$, and $\operatorname{Spec}(Z, V)=\Omega^{+}, \Omega^{-}, \Omega^{+} \cup\left\{1_{Z}\right\}, \Omega^{-} \cup\left\{1_{Z}\right\}$, or $\Omega^{+} \cup \Omega^{-}$.

Proof. Let $P=N_{G}(Z)$ and $C=C_{G}(Z)$. Assume that $\operatorname{Spec}(Z, V) \neq$ $\operatorname{IBr}_{\ell}(Z)$.

First suppose $G \neq \operatorname{Sp}_{2 n}(q)$, or $G=\operatorname{Sp}_{2 n}(q)$ but $q$ is even. Then $P / C \simeq$ $Z_{q-1}$ and $P$ acts transitively on $\Omega^{+} \cup \Omega^{-}=\operatorname{IBr}_{r}(Z) \backslash\left\{1_{Z}\right\}$. Since $\operatorname{Spec}(Z, V)$ contains at least one nontrivial linear character of $Z$, $\operatorname{Spec}(Z, V)=\Omega^{+} \cup \Omega^{-}$. Moreover, if $q=p$, then $G=\mathrm{SU}_{3}(p)$ by Theorem 2.8. Thus we arrive at (i) or (ii).

If $G=\mathrm{SL}_{2}(q)$, then we can check (v) directly.

So suppose that $G=\operatorname{Sp}_{2 n}(q), q$ is odd, and $n \geq 2$. In this case, any nontrivial element of $Z$ is a transvection, and $P$-orbits on $\Omega^{+} \cup \Omega^{-}$are $\Omega^{+}$ and $\Omega^{-}$. Moreover, $P$ contains a subgroup $P^{\prime}=Q L$, where $Q$ is a normal subgroup of extraspecial type of order $q^{2 n-1}$, with $Z=Z(Q)$, and $L=$ $\mathrm{Sp}_{2 n-2}(q)$. Fix a nontrivial linear character $\lambda$ occuring in $V$ and consider the $\lambda$-eigenspace $V^{\prime}$ for $Z$ in $V$. Then, as an $\mathbb{F} P^{\prime}$-module, $V^{\prime} \simeq W \otimes U$, where $W$ is an irreducible representation of degree $q^{n-1}$ (extending an irreducible representation of $Q$ ), and $U$ is a representation of $L=P^{\prime} / Q$ inflated to $P^{\prime}$. Moreover, $\left.W\right|_{L}$ is the sum of two Weil representations $W^{ \pm}$of degree $\left(q^{n-1} \pm 1\right) / 2$ if $r \neq 2$, and has three composition factors, one of dimension 1 and two, say $W_{1}, W_{2}$, of dimension $\left(q^{n-1}-1\right) / 2$ if $r=2$.

Since $n \geq 2, L$ contains a long-root subgroup $Z^{\prime}$ which is $G$-conjugate to $Z$. Let $n=2$. The above discussion shows that $\left|\operatorname{Spec}\left(Z^{\prime}, W\right)\right| \geq(q+1) / 2$. (For, if $r=2$ then $1_{Z^{\prime}} \in \operatorname{Spec}\left(Z^{\prime}, W\right)$, hence the claim follows. If $r \neq 2$ then $\left|\operatorname{Spec}\left(Z^{\prime}, W^{+}\right)\right|=(q+1) / 2$ and we are again done.) Thus $\mid \operatorname{Spec}\left(Z^{\prime}, W \otimes\right.$ $U) \mid \geq(q+1) / 2$, whence

$$
(q+1) / 2 \leq\left|\operatorname{Spec}\left(Z^{\prime}, W \otimes U\right)\right| \leq\left|\operatorname{Spec}\left(Z^{\prime}, V\right)\right|=|\operatorname{Spec}(Z, V)| .
$$

Therefore we arrive at (iv).

Finally, suppose $n \geq 3$. It suffices to show that $1_{Z^{\prime}} \in \operatorname{Spec}\left(Z^{\prime}, W \otimes U\right)$. Assume the contrary. If $r=2$, then clearly $1_{Z^{\prime}} \in \operatorname{Spec}\left(Z^{\prime}, W\right)$. If $r \neq 2$, then we can embed $Z^{\prime}$ into a standard subgroup $L^{\prime}$ of type $\operatorname{Sp}_{2}(q)$, and $\left.W^{+}\right|_{L^{\prime}}$ 
has a composition factor of dimension $(q+1) / 2$, whence $1_{Z^{\prime}} \in \operatorname{Spec}\left(Z^{\prime}, W\right)$. Therefore $1_{Z^{\prime}} \notin \operatorname{Spec}\left(Z^{\prime}, U\right)$. Due to (iv), $\operatorname{Spec}\left(Z^{\prime}, U\right)=\Omega^{+} \cup \Omega^{-}$. In this case, pick a character $\lambda_{c}$ with $c \in \mathbb{F}_{q}^{\times}$occuring in $\operatorname{Spec}\left(Z^{\prime}, W\right)$. Then $\lambda_{-c} \in$ $\operatorname{Spec}\left(Z^{\prime}, U\right)$, whence $1_{Z^{\prime}}=\lambda_{c} \lambda_{-c} \in \operatorname{Spec}\left(Z^{\prime}, W \otimes U\right)$, a contradiction.

Corollary 2.10. Let $G$ be a universal-type quasi-simple finite group of Lie type defined over $\mathbb{F}_{q}, q=p^{f}$, and $Z$ a long-root subgroup as above. Let $\mathbb{F}$ be an algebraically closed field of characteristic $\ell \neq p$ and $V$ a nontrivial irreducible $\mathbb{F} G$-module. Then either

(i) $\operatorname{Spec}(Z, V)$ contains at least two distinct characters which are not dual to each other, or

(ii) $G \in\left\{\mathrm{SL}_{2}(5), \mathrm{SU}_{3}(3), \mathrm{Sp}_{4}(3)\right\}$.

Proof. First assume that $q=2$. Then $Z$ is of order 2 and $Z$ is not central, hence $\left.V\right|_{Z}$ affords both linear characters of $Z$, and they are real and distinct, i.e., we are in (i). Next assume that $q=3$ and $G \neq \mathrm{SU}_{3}(3)$, $\mathrm{Sp}_{4}(3)$. By Lemma 2.9, $\operatorname{Spec}(Z, V)$ contains $1_{Z}$ and at least one more character, so we arrive at (i). Assume that $q=5$ and $G \neq \mathrm{SL}_{2}(5)$. By Lemma 2.9, $|\operatorname{Spec}(Z, V)| \geq 3$, which implies (i). Finally, if $q=4$ or $q \geq 7$, then $|\operatorname{Spec}(Z, V)| \geq 3$ by Lemma 2.9, and we again arrive at (i).

\section{The good case.}

We first treat the good cases where Proposition 2.3 applies, that is, if either $p \neq 2$ or $(\operatorname{type}(V), X) \notin\left\{\left(-, \widetilde{\Sigma}^{2}\right),\left(+, \widetilde{\Lambda}^{2}\right)\right\}$, where we obtain reasonably complete results.

Let $\mathbb{F}$ be an algebraically closed field of characteristic $\ell$. The main result of this section is the following:

Theorem 3.1. Let $G$ be a quasi-simple group with $S:=G / Z(G)$ being a finite group of Lie type in characteristic p. Suppose that $G$ has a nontrivial irreducible $\mathbb{F} G$-module $V$ such that $X(V)$ is irreducible, where $X=\widetilde{A}$ if $V$ is not self-dual, and $X=\widetilde{\Sigma}^{2}$ or $\widetilde{\Lambda}^{2}$ otherwise. Then one of the following holds.

(i) $S=\mathrm{S}_{2 n}(q), q=3,5,9, V$ is a Weil module of $\operatorname{Sp}_{2 n}(q)$ of degree $\left(q^{n} \pm 1\right) / 2$.

(ii) $S=\mathrm{U}_{n}(q), q=2,3$, and $V$ is a Weil module of $\mathrm{SU}_{n}(q)$ of degree $\left(q^{n}+q(-1)^{n}\right) /(q+1)$ or $\left(q^{n}-(-1)^{n}\right) /(q+1)$.

(iii) $S={ }^{3} D_{4}(3), F_{4}(2), F_{4}(3), E_{6}(2),{ }^{2} E_{6}(2),{ }^{2} E_{6}(3), E_{7}(2)$ or $E_{8}(2)$.

(iv) "Small groups": $S$ is $\mathrm{L}_{n}(q)$ as in Table 3.1 of [18], or as in Table 3.2.

(v) $p=2$ and $(\operatorname{type}(V), X) \in\left\{\left(-, \widetilde{\Sigma}^{2}\right),\left(+, \widetilde{\Lambda}^{2}\right)\right\}$.

Remark 3.3. The Weil modules of $\operatorname{Sp}_{2 n}(q), q=3,5,9$, and of $\mathrm{SU}_{n}(q)$, $q=2,3$, do indeed give irreducible examples. In characteristic $\ell=0$, this question has been resolved in $[\mathbf{1 9}]$ for $\operatorname{Sp}_{2 n}(q)$, and in $[\mathbf{2 0}, \mathbf{1 4}]$ for $\mathrm{SU}_{n}(q)$. 
Table 3.2. Non-generic examples.

\begin{tabular}{|l|rr|lll|}
\hline \multicolumn{1}{|c|}{$G$} & \multicolumn{1}{c|}{$\ell$} & $\operatorname{dim}(V)$ & $\widetilde{\Lambda}^{2}$ & $\widetilde{\Sigma}^{2}$ & $\widetilde{A}$ \\
\hline $\mathrm{U}_{3}(4)$ & $\neq 2$ & 12 & $\times$ & & \\
$3_{1} \cdot \mathrm{U}_{4}(3)$ & 2 & 6 & & & $\times$ \\
$6_{1} \cdot \mathrm{U}_{4}(3)$ & $\neq 2,3$ & 6 & & & $\times$ \\
$2 . \mathrm{U}_{6}(2)$ & $\neq 2$ & 56 & $\times$ & & \\
\hline $\mathrm{S}_{4}(4)$ & $\neq 2$ & 18 & $\times$ & & \\
$2 . \mathrm{S}_{6}(2)$ & $\neq 2$ & 8 & & $\times$ & \\
\hline $2 . \mathrm{O}_{8}^{+}(2)$ & $\neq 2$ & 8 & $\times$ & $\times$ & \\
\hline $2 .{ }^{2} B_{2}(8)$ & 5 & 8 & & $\times$ & \\
$G_{2}(3)$ & 2 & 14 & $\times$ & & \\
$G_{2}(3)$ & $\neq 2,3$ & 14 & $\times$ & $\times$ & \\
$2 . G_{2}(4)$ & $\neq 2$ & 12 & $\times$ & $\times$ & \\
${ }^{2} F_{4}(2)^{\prime}$ & $\neq 2$ & 26 & & & $\times$ \\
${ }^{3} D_{4}(2)$ & 3 & 25 & & $\times$ & \\
\hline
\end{tabular}

Proof. We start by making some obvious reductions. The corresponding universal-type group of Lie type is the universal cover for $S$, with a few exceptions. These exceptions as well as those groups emerging in Corollary 2.10(ii) can be handled directly using [12], and the arising examples are recorded in (iii) or (iv). Throughout the proof we will therefore assume that $V$ is a nontrivial (could be non-faithful) irreducible module of a universal-type group $G$ of Lie type defined over a field $\mathbb{F}_{q}$ of characteristic $p$ and satisfying Corollary 2.10(i). Moreover, we can and will assume that $G$ is not a special linear group, since that case has been treated in [18].

1) We will apply Proposition 2.3 to a long-root subgroup $Z$ of $G$. If $C:=C_{G}(Z)$ and $N:=N_{G}(Z)$, then $N / C$ is a cyclic group, of order $(q-1) / 2$ if $G=\operatorname{Sp}_{2 n}(q)$ with $q$ odd, $q^{2}-1$ if $G={ }^{2} B_{2}\left(q^{2}\right),{ }^{2} G_{2}\left(q^{2}\right),{ }^{2} F_{4}\left(q^{2}\right)$, and $q-1$ otherwise.

By our initial reductions we may assume by Corollary 2.10 that $(V, Z)$ satisfies the assumptions of Proposition 2.3. According to Proposition 2.3(i) and (ii), $\operatorname{dim} X(V) \leq(G: N)$. Since $\operatorname{dim} X(V) \geq \operatorname{dim}(V)(\operatorname{dim}(V)-1) / 2-2$, this implies that

$$
\operatorname{dim}(V) \leq \frac{1}{2}+\sqrt{2(G: N)+\frac{17}{4}} .
$$

2) We assume that $G$ is one of the groups in the following table, where moreover $(n, q) \notin\{(3,3),(3,4),(4,2),(4,3),(6,2)\}$ for $G=\operatorname{SU}_{n}(q),(n, q) \notin$ 
$\{(2,2),(2,3),(3,2)\}$ for $G=\operatorname{Sp}_{2 n}(q)$. Then $N$ is as indicated, cf. [13]. Here, for an integer $m,[m]$ is a certain group of that order.

Table 3.5. The group $N$.

\begin{tabular}{|ll|l|}
\hline \multicolumn{1}{|c|}{ condition } & \multicolumn{1}{c|}{$N$} \\
\hline $\mathrm{SU}_{n}(q)$ & $n \geq 3$ & {$\left[q^{2 n-3}\right] \cdot\left(\mathrm{SU}_{n-2}(q) \cdot Z_{q^{2}-1}\right)$} \\
$\operatorname{Sp}_{2 n}(q)$ & & {$\left[q^{2 n-1}\right] \cdot\left(\mathrm{Sp}_{2 n-2}(q) \cdot Z_{q-1}\right)$} \\
$\operatorname{Spin}_{2 n+1}(q)$ & $n \geq 3,(n, q) \neq(3,3)$ & {$\left[q^{4 n-5}\right] \cdot\left(\mathrm{SL}_{2}(q) \times \mathrm{Spin}_{2 n-3}(q)\right) \cdot Z_{q-1}$} \\
$\operatorname{Spin}_{2 n}^{\epsilon}(q)$ & $n \geq 4,(n, q) \neq(4,2)$ & {$\left[q^{4 n-7}\right] \cdot\left(\mathrm{SL}_{2}(q) \times \operatorname{Spin}_{2 n-4}^{\epsilon}(q)\right) \cdot Z_{q-1}$} \\
${ }^{2} B_{2}\left(q^{2}\right)$ & $q^{2}>8$ & {$\left[q^{4}\right] \cdot Z_{q^{2}-1}$} \\
${ }^{2} G_{2}\left(q^{2}\right)$ & $q^{2} \geq 27$ & {$\left[q^{6}\right] \cdot Z_{q^{2}-1}$} \\
$G_{2}(q)$ & $q \geq 5$ & {$\left[q^{5}\right] \cdot\left(\mathrm{SL}_{2}(q) \cdot Z_{q-1}\right)$} \\
${ }^{3} D_{4}(q)$ & $q \geq 3$ & {$\left[q^{9}\right] \cdot\left(\mathrm{SL}_{2}\left(q^{3}\right) \cdot Z_{q-1}\right)$} \\
$F_{4}(q)$ & $q \geq 3$ & {$\left[q^{15}\right] \cdot\left(\mathrm{Sp}_{6}(q) \cdot Z_{q-1}\right)$} \\
${ }^{2} F_{4}\left(q^{2}\right)$ & $q^{2}>2$ & {$\left[q^{22}\right] \cdot\left({ }^{2} B_{2}\left(q^{2}\right) \cdot Z_{q^{2}-1}\right)$} \\
$E_{6}(q)$ & & {$\left[q^{21}\right] \cdot\left(\mathrm{SL}_{6}(q) \cdot Z_{q-1}\right)$} \\
${ }^{2} E_{6}(q)$ & $q \geq 3$ & {$\left[q^{21}\right] \cdot\left(\mathrm{SU}_{6}(q) \cdot Z_{q-1}\right)$} \\
$E_{7}(q)$ & & {$\left[q^{33}\right] \cdot\left(\mathrm{Spin}_{12}^{+}(q) \cdot Z_{q-1}\right)$} \\
$E_{8}(q)$ & & {$\left[q^{57}\right] \cdot\left(E_{7}(q) \cdot Z_{q-1}\right)$} \\
\hline
\end{tabular}

Let $d$ be the smallest degree of nontrivial irreducible projective representations of $G$ in cross-characteristics. Lower bounds on $d$ were given in $[13,23,6,9,10]$. The obvious lower bound $\operatorname{dim}(V) \geq d$ and the upper bound (3.4) imply $\sqrt{2(G: N)+17 / 4}+1 / 2 \geq d$. The last inequality can hold only when $G$ is one of the following groups:

$$
\begin{aligned}
& \mathrm{SU}_{n}(2), \mathrm{SU}_{n}(3), \mathrm{Sp}_{2 n}(3), \mathrm{Sp}_{2 n}(5), \mathrm{Sp}_{2 n}(7), \mathrm{Sp}_{2 n}(9), \mathrm{O}_{2 n}^{ \pm}(2), \\
& \operatorname{Spin}_{8}^{+}(3),{ }^{3} D_{4}(3), F_{4}(3), E_{6}(2),{ }^{2} E_{6}(3), E_{7}(2), E_{8}(2) .
\end{aligned}
$$

(A typical calculation is given in the case of $G=E_{8}(q)$ as follows: $(G: N)=$ $\left(q^{30}-1\right)\left(q^{12}+1\right)\left(q^{10}+1\right)\left(q^{6}+1\right) /(q-1)$ and $d \geq q\left(q^{12}+1\right)\left(q^{10}+1\right)\left(q^{6}+1\right)-3$ (cf. [10]), hence $\sqrt{2(G: N)+17 / 4}+1 / 2<d$ if $q \geq 3$.)

3) To complete the proof of the theorem, we have to analyze the groups singled out in (3.6).

Assume $G=\mathrm{SU}_{n}(q)$ and $q=2,3$. Then (3.4) implies that $\operatorname{dim} V<2 d$. By [7], Th. 16, $V$ is one of the Weil modules of $G$. 
Assume $G=\operatorname{Sp}_{2 n}(q), n \geq 2, q$ odd and $q \leq 9$. If $q \geq 5$, then

$$
\begin{aligned}
\operatorname{dim} V & \leq \sqrt{2(G: N)+17 / 4}+1 / 2 \\
& =\sqrt{2\left(q^{2 n}-1\right) /(q-1)+17 / 4}+1 / 2<q^{n}-1 .
\end{aligned}
$$

Suppose $q=3$. Since $n \geq 3$, $\operatorname{Spec}(Z, V) \ni 1_{Z}$ by Corollary 2.10. By Proposition 2.3(i), $X(V)$ is a constituent of a reduction modulo $\ell$ of the complex character $\rho:=\left(1_{N}\right) \uparrow G$. Observe that $\rho$ is the sum of the trivial character, a character of degree $\left(q^{n}+1\right)\left(q^{n}-q\right) / 2(q-1)$, and a character of degree $\left(q^{n}-1\right)\left(q^{n}+q\right) / 2(q-1)$. Therefore $\operatorname{dim} X(V) \leq\left(q^{n}-1\right)\left(q^{n}+\right.$ q) $/ 2(q-1)$, whence $\operatorname{dim} V<q^{n}-1$. Thus in all cases $\operatorname{dim} V<q^{n}-1$. By [6], Thm. 9.9.2, $\operatorname{dim} V=\left(q^{n} \pm 1\right) / 2$. This implies by the main result of [5] that $V$ is a Weil module. Now apply Prop. 5.5 in $[\mathbf{1 9}]$ and use that a Weil module is self-dual if and only if $q \equiv 1(\bmod 4)$.

4) Assume that $G=\mathrm{O}_{2 n}^{\epsilon}(2)$ and $n \geq 4$. Consider the natural module $\mathbb{F}_{2}^{2 n}$ for $G$ and the stabilizer $P$ of an isotropic vector in this module. Then $P=U \cdot L$, where $U$ is a normal elementary abelian subgroup of order $2^{2 n-2}$ and $L=\mathrm{O}_{2 n-2}^{\epsilon}(2)$. The group $L$ acts on $\operatorname{IBr}_{\ell}(U)$ with three orbits, of length $1, a:=\left(2^{n-2}+\epsilon 1\right)\left(2^{n-1}-\epsilon 1\right)$, and $b:=2^{n-2}\left(2^{n-1}-\epsilon 1\right)$, respectively. Observe that

$$
\max \{a, b\}<d \leq \operatorname{dim} V \leq \sqrt{2(G: N)+17 / 4}+1 / 2<2 \min \{a, b\} .
$$

This shows that $L$ has at least two different orbits on $\operatorname{Spec}(U, V)$. Each orbit gives rise to a direct summand in the $P$-module $V$. Thus we can write $\left.V\right|_{P}=V_{1} \oplus V_{2}$ with $V_{1}$ and $V_{2}$ having no common composition factors. Here we have $p=2$, so due to our assumption $V$ is of type + if $X=\widetilde{\Sigma}^{2}$ and $V$ is of type - if $X=\widetilde{\Lambda}^{2}$. Arguing as in the proof of Proposition 2.3(i) (with $P$ in place of $C)$, we see that $X(V) \hookrightarrow\left(1_{P}\right) \uparrow^{G}$, in particular, $\operatorname{dim} X(V) \leq$ $(G: P)$. Thus

$$
\operatorname{dim} V \leq \sqrt{2(G: P)+\frac{17}{4}}+\frac{1}{2}=\sqrt{2\left(2^{n}-\epsilon 1\right)\left(2^{n-1}+\epsilon 1\right)+\frac{17}{4}}+\frac{1}{2},
$$

whence $\operatorname{dim} V \leq 2^{n}+1<d$, a contradiction.

5) Let $G=\operatorname{Spin}_{8}^{+}(3)$. In this case (3.4) implies that $\operatorname{dim} V \leq 270$. We can embed $\operatorname{Spin}_{7}(3)$ in $G$. According to [12], the dimension of any faithful representation of $\operatorname{Spin}_{7}(3)$ in characteristic $\neq 2,3$ is at least 520. From this it follows that $V$ is actually a representation of $G / Z(G)=\mathrm{O}_{8}^{+}(3)$. The modular character tables of this group are known.

For the groups in Case (iii) of Theorem 3.1, (3.4) implies at least the upper bounds for $\operatorname{dim}(V)$ given in the following table: 


\begin{tabular}{|l|r|r|}
\hline$G$ & $l(G)$ & $\operatorname{dim}(V) \leq$ \\
\hline${ }^{3} D_{4}(3)$ & 216 & 231 \\
$F_{4}(3)$ & 5832 & 6601 \\
$E_{6}(2)$ & 1536 & 3188 \\
${ }^{2} E_{6}(3)$ & 157464 & 175030 \\
$E_{7}(2)$ & 98304 & 192927 \\
$E_{8}(2)$ & 402653184 & 765625740 \\
\hline
\end{tabular}

Here, $l(G)$ denotes the lower bound for nontrivial irreducible representations in cross-characteristic from $[\mathbf{1 3}, \mathbf{2 3}, \mathbf{1 0}]$. If $\ell=0$, then each $G$ in the table has exactly one nontrivial irreducible module $V$ of the indicated dimension, cf. [17], and one can check that $\operatorname{dim}(X(V))$ does not divide $|G|$. The complex characters of $2 . F_{4}(2)$ and $2 .{ }^{2} E_{6}(2)$ can be checked by inspecting the character table and using GAP. Finally, let $G=3 .{ }^{2} E_{6}(2)$ or $6 .{ }^{2} E_{6}(2)$, and $V$ a faithful irreducible $\mathbb{C} G$-module. Then $V$ is not self-dual, so we need to consider only $\widetilde{A}(V)$. Let $z$ be an inverse image in $G$ of a long-root element and let $Z=\langle z\rangle$. In order to apply Proposition 2.3, we need to show that $z$ has at least two non-conjugate eigenvalues in $V$. Suppose not, then $z$ has exactly two eigenvalues say $\alpha$ and $\alpha^{-1}$. Write $V=U \oplus W$ for the corresponding eigenspaces. Then $A(V)=A(U) \oplus A(W) \oplus \ldots$ contains at least two copies of the trivial $C$-module (with $C:=C_{G}(Z)$ ), so $\widetilde{A}(V)$ contains $1_{C}$. Thus in any case we have $\operatorname{dim}(V) \leq \sqrt{(G: C)+1}$, i.e., $\operatorname{dim}(V) \leq 1991$. According to [17], there is no such faithful $G$-module.

Thus we have completed the good case for $\ell=0$.

\section{Lower bounds for representations of ${ }^{3} D_{4}(q)$ and ${ }^{2} E_{6}(q)$.}

In this section we improve the Landázuri-Seitz-Zalesskii bounds for the smallest degree of a nontrivial representation in non-defining characteristic for the twisted exceptional groups ${ }^{3} D_{4}(q)$ and ${ }^{2} E_{6}(q)$. Our method is a direct extension of the one used by Hoffman [10] for the non-twisted groups of type $E_{n}$.

Theorem 4.1. Let $V$ be a nontrivial irreducible representation of ${ }^{3} D_{4}(q)$ in characteristic $\ell \backslash q$. Then

$$
\operatorname{dim}(V) \geq q\left(q^{4}-q^{2}+1\right)-1 .
$$

Proof. Let $N=Q . \mathrm{SL}_{2}\left(q^{3}\right) \cdot Z_{q-1}$ be a maximal parabolic subgroup of ${ }^{3} D_{4}(q)$ with special unipotent radical $Q$ of order $q^{1+8}$. The restriction of $V$ to $Q$ splits into $V_{1} \oplus V_{2} \oplus V_{3}$ where $V_{1}=C_{V}(Q), V_{2}$ is the part on which $Q$ acts by nontrivial linear characters, and $\left.V_{3}=[Z(Q), V)\right]$. Any non-linear irreducible 
representation of $Q$ has degree $q^{4}$, and all these are conjugate by an element of order $q-1$ in $L$. Thus $\operatorname{dim}\left(V_{3}\right)=m q^{4}(q-1)$ for some positive $m$.

Let $g$ be a long root element in $Z(Q)$. Then the Brauer character on $V_{3}$ takes value $-m q^{4}$ on $g$. On the other hand, it vanishes on any long root element in $Q \backslash Z(Q)$ by [13], Lemma 2.3. Since all long root elements are conjugate in ${ }^{3} D_{4}(q)$ and $Q$ acts trivially on $V_{1}$ we conclude that $V_{2} \neq 0$.

The space $U$ of linear characters of $Q$ is isomorphic to $M \otimes M^{F} \otimes M^{F^{2}}$ as $\mathrm{SL}_{2}\left(q^{3}\right)$-module, where $M$ is the natural $\mathrm{SL}_{2}$-module and $F$ is the Frobenius map of $\mathbb{F}_{q^{3}} / \mathbb{F}_{q}$. Clearly $\mathrm{SL}_{2}\left(q^{3}\right)$ has no fixed points on $U$, nor has the maximal subgroup $\mathrm{SL}_{2}(q)$. The Borel subgroup stabilizes a line, hence a subgroup of index $q-1$ fixes a vector, giving an orbit of length $\left(q^{3}+1\right)(q-1)$. All other subgroups of $\mathrm{SL}_{2}\left(q^{3}\right)$ have index at least $\frac{1}{2} q^{2}$ times that large. Hence $\operatorname{dim}\left(V_{2}\right) \geq\left(q^{3}+1\right)(q-1)$, and we obtain

$\operatorname{dim}(V) \geq \operatorname{dim}\left(V_{2}\right)+\operatorname{dim}\left(V_{3}\right) \geq q^{4}(q-1)+\left(q^{3}+1\right)(q-1)=q\left(q^{4}-q^{2}+1\right)-1$.

Note that, by the results of Lusztig, ${ }^{3} D_{4}(q)$ has a complex irreducible unipotent character of degree $q\left(q^{4}-q^{2}+1\right)$. Moreover, Harish-Chandra induction of projectives from the Levi subgroup $L=\mathrm{SL}_{2}\left(q^{3}\right) \cdot Z_{q-1}$ shows that in characteristic $\ell \mid(q+1)$ this splits off a trivial composition factor, hence the above result is best possible. In particular our bound is better than the bound $q^{3}\left(q^{2}-1\right)$ given in [13]. In the case $q$ odd and $\ell \neq 2$ an alternative proof of Theorem 4.1 using generalized Gelfand-Graev characters was given in [17], 4.4.

Theorem 4.2. Let $V$ be a nontrivial irreducible representation of ${ }^{2} E_{6}(q)_{s c}$ in characteristic $\ell \nmid q$. Then

$$
\operatorname{dim}(V) \geq q\left(q^{4}+1\right)\left(q^{6}-q^{3}+1\right)-2 .
$$

Proof. Let $N=Q . \mathrm{SU}_{6}(q) \cdot Z_{q-1}$ be a maximal parabolic subgroup of ${ }^{2} E_{6}(q)$ with special unipotent radical $Q$ of order $q^{1+20}$. We proceed as in the previous proof. Now $V_{3}:=[Z(Q), V]$ has dimension $m q^{10}(q-1)$ for some positive $m$, and consideration of values of Brauer characters on long root elements shows that the linear part $V_{2}$ is nontrivial.

In order to determine the orbits of the Levi factor $L:=\mathrm{SU}_{6}(q) \cdot Z_{q-1}$ on the linear characters of $Q$ we first look at the case of the algebraic group of type $E_{6}$. By the result of Hoffman in the case of the untwisted group [10], Sect. 2, the Levi subgroup of type $\mathrm{SL}_{6}$ has five nontrivial orbits on the linear characters of the corresponding unipotent radical. Representatives for these are known explicitly. Taking fixed points under the twisted Frobenius 
morphism of $E_{6}$ then yields that in our case the nontrivial orbits have lengths

$$
\begin{aligned}
& \left(q^{2}-1\right)\left(q^{3}+1\right)\left(q^{5}+1\right), \quad q^{2}\left(q^{3}+1\right)\left(q^{5}+1\right)\left(q^{6}-1\right) /(q+1), \\
& q^{4}\left(q^{4}-1\right)\left(q^{5}+1\right)\left(q^{6}-1\right), \quad q^{9}(q-1)\left(q^{2} \mp 1\right)\left(q^{3} \pm 1\right)\left(q^{5}+1\right) / 2 .
\end{aligned}
$$

Thus we have $\operatorname{dim}\left(V_{2}\right) \geq\left(q^{2}-1\right)\left(q^{3}+1\right)\left(q^{5}+1\right)$, the length of the shortest orbit.

By Lusztig's classification there exists a unipotent complex module $\widetilde{V}$ of $G$ of dimension $q\left(q^{4}+1\right)\left(q^{6}-q^{3}+1\right)$. The preceding argument shows that this satisfies

$$
\begin{aligned}
\operatorname{dim}\left(C_{\widetilde{V}}(Q)\right) & =\operatorname{dim}(\widetilde{V})-\left(q^{2}-1\right)\left(q^{3}+1\right)\left(q^{5}-1\right)-q^{10}(q-1) \\
& =\left(q^{6}+2 q+1\right) /(q+1) .
\end{aligned}
$$

The permutation character of the Weyl group of $E_{6}$ on the cosets of $\mathfrak{S}_{6}$ has five constituents, hence (by Harish-Chandra theory) $L$ has at most five trivial composition factors on $C_{\widetilde{V}}(Q)$. Any nontrivial $L$-composition factor of $C_{\widetilde{V}}(Q)$ has dimension at least $\left(q^{6}-1\right) /(q+1)$ (see for example [7]). Comparison of the Brauer characters of $V$ and $\widetilde{V}$ on long root elements [10] shows that hence $L$ must also act nontrivially on $V_{1}=C_{V}(Q)$, whence $\operatorname{dim}\left(V_{1}\right) \geq\left(q^{6}-1\right) /(q+1)$. In conclusion we obtain

$$
\begin{aligned}
\operatorname{dim}(V) & =\operatorname{dim}\left(V_{1}\right)+\operatorname{dim}\left(V_{2}\right)+\operatorname{dim}\left(V_{3}\right) \\
& \geq\left(q^{6}-1\right) /(q+1)+\left(q^{2}-1\right)\left(q^{3}+1\right)\left(q^{5}+1\right)+q^{10}(q-1) \\
& =q\left(q^{4}+1\right)\left(q^{6}-q^{3}+1\right)-2 .
\end{aligned}
$$

As stated in the proof, there exists a complex unipotent representation of ${ }^{2} E_{6}(q)$ of degree $q\left(q^{4}+1\right)\left(q^{6}-q^{3}+1\right)$. The lower bound given in [13] was $q^{9}\left(q^{2}-1\right)$.

\section{The bad case.}

In this section we deal exclusively with the bad case, that is, where $p=2$ (so $\ell$ is odd) and (type $(V), X) \in\left\{\left(-, \widetilde{\Sigma}^{2}\right),\left(+, \widetilde{\Lambda}^{2}\right)\right\}$. In particular we may assume here that $V$ carries a nondegenerate bilinear form and $X(V)=Y(V)$.

We are able to eliminate all classical groups over fields $\mathbb{F}_{q}$ with $q \geq 16$ ( $q \geq 8$ if $G$ is not symplectic) and to derive upper bounds for $\operatorname{dim}(V)$ for the remaining $q$. These bounds are very close to the known lower bounds $l(G)$ for the dimension of nontrivial representations of $G$ in cross characteristic, the worst case occurring for $(q, \ell)=(2,3)$. For exceptional groups not of type $F_{4}$ we show that necessarily $X=\widetilde{\Lambda}^{2}$ and $\operatorname{dim}(V)<2 l(G)$. Finally, for ${ }^{2} F_{4}$ and $F_{4}$ we obtain that $\operatorname{dim}(V) \leq 4 q l(G)$. 


\section{A. Groups of small rank.}

We first show that certain small rank groups do not lead to examples. For

$$
G \in\left\{\mathrm{SU}_{3}(q), \mathrm{Sp}_{4}(q),{ }^{2} B_{2}\left(q^{2}\right)\right\},
$$

$q$ even, we may argue as follows. A lower bound for the dimension of an irreducible nontrivial $\mathbb{F} G$-module is $\left(q^{3}-q\right) /(q+1), q(q-1)^{2} / 2, q / \sqrt{2}\left(q^{2}-1\right)$ (for $q^{2}>8$ ) by [23]. On the other hand, the largest degree of an irreducible complex representation for $G$ is given by $\left(q^{2}-1\right)(q+1),(q+1)^{2}\left(q^{2}+1\right)$, $\left(q^{2}-1\right)\left(q^{2}+\sqrt{2} q+1\right)$ respectively (for example by Deligne-Lusztig theory). The assumption that $X(V)$ is irreducible now leads to a contradiction for $q \geq 8, q \geq 8, q^{2}>8$ respectively. The tables of Brauer characters of the remaining groups are contained in $[\mathbf{1 2}]$ and allow to verify that no examples arise.

Lemma 5.1. Let $G=G_{2}(q), q \geq 8$ even, $\ell \neq 2$, and $V$ a self-dual absolutely irreducible faithful $\mathbb{F} G$-module. Then $X(V)$ is reducible for $X \in\left\{\widetilde{\Lambda}^{2}, \widetilde{\Sigma}^{2}\right\}$.

Proof. The largest ordinary character degree of $G$ equals $(q+1)\left(q^{2}+q+\right.$ $1)\left(q^{3}+1\right)$, thus $\operatorname{dim}(V)$ is bounded above by $2 q^{3}$. By the known decomposition numbers for $G_{2}(q)$ [8] this implies that $V$ is the largest irreducible constituent of the ordinary irreducible $\hat{V}$ of degree $q^{3}+\epsilon$ (where $q \equiv \epsilon$ $(\bmod 3))$. Moreover $\hat{V}$ remains irreducible in positive characteristic unless $\ell=3$ and $\epsilon=1$, and in the latter case it splits off one trivial composition factor. The ordinary character table of $G_{2}(q)$ is known, and it can be verified with Chevie [2] that both the symmetric and the alternating square of the character $\chi$ of $\hat{V}$ contain several irreducible constituents in characteristic 0 , hence in characteristic $\ell \neq 3$.

If $\ell=3$, one checks again in characteristic 0 that the tensor product of $\chi-1$ with itself decomposes positively, and neither alternating nor symmetric square can be irreducible.

\section{B. Unitary groups.}

Recall that $\mathrm{SU}_{3}(q)$ was handled in part A. For unitary groups in dimension at least 4 we first assume that $q \neq 2$.

Proposition 5.2. Let $G$ be a covering group of $\mathrm{U}_{n}(q), n \geq 4,2 \neq q$ even, $\ell \neq 2$ and $V$ a self-dual absolutely irreducible faithful $\mathbb{F} G$-module. Then $X(V)$ is reducible for $X \in\left\{\Lambda^{2}, \mathrm{Sym}^{2}\right\}$.

Proof. We first claim that $V$ must be the $\ell$-modular reduction of a Weil representation by applying gap results for low-dimensional irreducible representations and a recognition theorem for Weil representations proved in 
[7]. Thus assume that $V$ can not be obtained in this way. Then we have

$$
\operatorname{dim}(V) \geq \begin{cases}\left(q^{2}-q+1\right)\left(q^{2}+1\right)-1 & \text { if } n=4 \\ q^{n-2}(q-1)\left(q^{n-2}-q\right) /(q+1) & \text { if } n \geq 5 \text { is odd } \\ q^{n-2}(q-1)\left(q^{n-2}-1\right) /(q+1) & \text { if } n \geq 6 \text { is even }\end{cases}
$$

by $[7]$ Th. 16 .

We now derive an upper bound for $\operatorname{dim}(X(V))$. If $n=4,5$ we just take the largest degree $q(q+1)^{3}\left(q^{2}+1\right)$, resp. $(q+1)^{3}\left(q^{2}+1\right)\left(q^{5}+1\right)$ of an ordinary irreducible character. This contradicts the lower bound. For $n \geq 6$ let $N$ be the maximal parabolic subgroup considered in Section 3, the stabilizer of an isotropic 1-space, with Levi subgroup $L$ such that $L^{\prime}=\mathrm{SU}_{n-2}(q)$ and special unipotent radical $Q$ of type $q^{1+2(n-2)}$. The restriction of $V$ to $Q$ thus splits into a direct sum of $C_{V}(Q)=V_{1}, V_{3}=[Z(Q), V]$ and the part $V_{2}$ on which $Q$ acts by nontrivial linear characters. Furthermore, $V_{3}$ splits into the isotypic components $V_{\psi}$ for the $q-1$ non-linear irreducible characters $\psi$ of $Q$ of degree $q^{n-2}$. Let $M$ be an isotypic $Q$-component on $V_{3}$ of dimension $d q^{n-2}$. By Lemma 2.3 and Proposition 2.4 in [19] $M \otimes M$ contains an $N$-submodule of dimension $d^{2}$. Since $V$ is self-dual and $2 \mid q$ we deduce that $X(V)$ also contains an $N$-submodule of dimension $d(d \pm 1) / 2$ (the sign depending on $X$ and the type of $Q$ ). If $d>1$, this gives the upper bound $\operatorname{dim}(X(V)) \leq[G: N] d(d \pm 1) / 2$, while on the other hand clearly $\operatorname{dim}(V) \geq d q^{n-2}(q-1)$, which leads to a contradiction for $q \geq 4$. Thus all $Q$-isotypic parts of $V_{3}$ are irreducible, and $\operatorname{dim}\left(V_{3}\right)=q^{n-2}(q-1)$.

We next estimate the dimension of $V_{2}$. Let $\mu$ denote the Brauer character of $V$ and $\mu_{i}$ the Brauer character of $V_{i}, i=2,3$. Let $g \in Z(Q)$ be a central involution of $Q$. Since $V_{3}$ contains all non-linear characters of $Q$ exactly once, $\mu_{3}(g)=-q^{n-2}$ and $\mu(g)=\operatorname{dim}(V)-\operatorname{dim}\left(V_{3}\right)-q^{n-2}=\operatorname{dim}(V)-q^{n-1}$ since $Z(Q)$ acts trivially on $V_{1} \oplus V_{2}$. By the main result of $[\mathbf{3}], \mu(g) / \operatorname{dim}(V) \leq 3 / 4$, hence $\operatorname{dim}(V) \leq 4 q^{n-1}$. But this contradicts the lower bound given above.

Thus $V$ is the $\ell$-modular reduction of a complex Weil representation $\hat{V}$ and has dimension $\left(q^{n}+(-1)^{n} q\right) /(q+1)$ or $\left(q^{n}-(-1)^{n}\right) /(q+1)$ (see [7], Th. 16). In particular $X(V)$ is reducible unless the complex representation $X(\hat{V})$ is irreducible. The latter cannot happen according to [14], Prop. 3.8.

Proposition 5.3. Let $G$ be a covering group of $\mathrm{U}_{n}(2), n \geq 5, \ell \neq 2$ and $V$ a self-dual absolutely irreducible faithful $\mathbb{F} G$-module. Then $X(V)$ is reducible unless possibly if

$$
\operatorname{dim}(X(V)) \leq \begin{cases}2^{2 n-4}\left(2^{n}-(-1)^{n}\right)\left(2^{n-1}+(-1)^{n}\right) / 9 & \text { if } \ell \neq 3, \\ 2^{n}\left(2^{n}-(-1)^{n}\right)\left(2^{n-1}+(-1)^{n}\right)\left(2^{n-3}+1\right) / 3 & \text { if } \ell=3\end{cases}
$$

Proof. The cases $n \leq 6$ can be checked directly. Hence we may assume $n \geq 7$ and consider $V$ as a $G$-module with $G=\mathrm{SU}_{n}(2)$. 
For $\ell \neq 3$ consider the subgroup $H=\mathrm{SU}_{2}(2) \times \mathrm{SU}_{n-2}(2)$. The first factor is isomorphic to the symmetric group $\mathfrak{S}_{3}$. The nontrivial eigenspaces for an element $g$ of order 3 in this factor are dual to each other as $C$-modules, where $C:=C_{G}(g)$. Thus $X(V)$ contains a trivial $C$-module and so a 1dimensional module for $M:=N_{G}(\langle g\rangle)$. Observe that $|M|=3|H|$. This leads to the estimate

$$
\operatorname{dim}(X(V)) \leq(G: M)=2^{2 n-4} \frac{\left(2^{n}-(-1)^{n}\right)\left(2^{n-1}+(-1)^{n}\right)}{9} .
$$

Now assume that $\ell=3$. We first prove a crude upper bound for $\operatorname{dim}(X(V))$ as follows. Let $H=\mathrm{SU}_{3}(2) \times \mathrm{SU}_{n-3}(2)$. We may now restrict to the eigenspaces of order-4 elements in the quaternion group contained in the first factor to obtain a trivial composition factor for $\mathrm{SU}_{n-3}(2)$ in the socle of $X(V)$. The 3-modular Brauer characters of $\mathrm{SU}_{3}(2)$ have degree at most 2, and we conclude

$$
\operatorname{dim}(X(V)) \leq 2^{3 n-8} \frac{\left(2^{n}-(-1)^{n}\right)\left(2^{n-1}+(-1)^{n}\right)\left(2^{n-2}-(-1)^{n}\right)}{27}
$$

To improve this bound, as above let $N=Q . L$ be a maximal parabolic subgroup of $G$ with $L^{\prime}=\mathrm{SU}_{n-2}(2)$. Assume first that $C_{V}(Q)=: V_{1} \neq 0$ and let $S$ be an $L$-composition factor of $V_{1}$. By [5], Lemma 4.2(iii), this occurs again as an $L$-composition factor of $[V, Q]$. So both the symmetric and the alternating square of $V$ contain a trivial $L$-composition factor and

$$
\operatorname{dim}(X(V)) \leq[G: L]=2^{2 n-3}\left(2^{n}-(-1)^{n}\right)\left(2^{n-1}+(-1)^{n}\right) / 3 .
$$

Otherwise, as $V$ is faithful for $G$, the center of the extraspecial group $Q$ doesn't act by scalars. So there exists a nontrivial linear character $\lambda$ of $Q$ such that the corresponding isotypic component $V_{\lambda}$ of $V$ is nonzero. Denote by $I_{\lambda}$ the stabilizer of $\lambda$ in $L$. Then $I_{\lambda}$ stabilizes two further characters $\lambda^{\prime}, \lambda^{\prime \prime}$ of $Q$, and $\lambda, \lambda^{\prime}, \lambda^{\prime \prime}$ are conjugate by an element of order three in the normalizer of $I_{\lambda}$. Write $\widetilde{Q}$ for the intersection of the kernels of $\lambda, \lambda^{\prime}, \lambda^{\prime \prime}$, a subgroup of $Q$ of index 4 . We distinguish two cases according to the type of $\lambda$.

If $\lambda$ is anisotropic, then $I_{\lambda}=\mathrm{SU}_{n-3}(2)$. If $3 \not \supset n$ then $\lambda, \lambda^{\prime}, \lambda^{\prime \prime}$ are already conjugate in the centralizer of $I_{\lambda}$. In particular the selfdual $\widetilde{Q} \cdot I_{\lambda}$-modules $V_{\lambda}, V_{\lambda}^{\prime}$ are equivalent, so $X(V)$ contains a trivial $\widetilde{Q} \cdot I_{\lambda}$-composition factor in the socle. This gives the upper bound

$$
\operatorname{dim}(X(V)) \leq\left[G: \widetilde{Q} \cdot I_{\lambda}\right] \leq 4.2^{n-3}\left(2^{n-2}+1\right)\left(2^{n}-(-1)^{n}\right)\left(2^{n-1}+(-1)^{n}\right) / 3 .
$$

When $3 \mid n$ the trivial and the (at most two) Weil representations of $I_{\lambda}$ are invariant under the diagonal outer automorphism of order 3 . Thus if the socle of $V_{\lambda}$ contains one of these representation, then the same is true for $V_{\lambda^{\prime}}$, and we conclude as in the previous case. Otherwise, by [7], Th. 16, we 
have $\operatorname{dim}\left(V_{\lambda}\right) \geq 2^{n-4}\left(2^{n-6}-1\right) / 3$, so

$$
\operatorname{dim}(V) \geq\left[L: I_{\lambda}\right] \operatorname{dim}\left(V_{\lambda}\right) \geq 2^{n-3}\left(2^{n-2}-(-1)^{n}\right) 2^{n-4}\left(2^{n-6}-1\right) / 3,
$$

violating the upper bound (5.4) if $n \geq 10$, respectively larger than the square root of the largest character degree if $n=9$ (note that $3 \mid n$ ).

It remains to consider the case of isotropic $\lambda$, with

$$
I_{\lambda}=2^{1+2(n-4)} \cdot \mathrm{GU}_{n-4}(2) .
$$

If the unipotent radical $R$ of $I_{\lambda}$ acts trivially on $V_{\lambda}$, we may argue as in the previous case, either obtaining the upper bound

$$
\operatorname{dim}(X(V)) \leq\left[G: \widetilde{Q} \cdot I_{\lambda}\right] \leq 4.2^{n-2}\left(2^{n-3}+1\right)\left(2^{n}-(-1)^{n}\right)\left(2^{n-1}+(-1)^{n}\right) / 3
$$

or a contradiction to (5.4) respectively to the largest degree of an ordinary irreducible character when $n=9$.

On the other hand, if $V_{\lambda}$ contains a nontrivial linear character $\mu$ of $R$, then

$$
\begin{aligned}
\operatorname{dim}(V) & \geq\left[L: I_{\lambda}\right] \operatorname{dim}\left(V_{\lambda}\right) \\
& \geq\left[L: I_{\lambda}\right]\left[I_{\lambda}: I_{\lambda \mu}\right]=2^{n-2}\left(2^{n-3}+1\right) 2^{n-4}\left(2^{n-5}+1\right),
\end{aligned}
$$

with the stabilizer $I_{\lambda \mu}$ in $\mathrm{GU}_{n-4}(2)$ of $\mu$, too large compared to (5.4) for $n \geq 7$.

If finally $V_{\lambda}$ contains the faithful character of $R$, then at least

$$
\operatorname{dim}(V) \geq\left[G: \widetilde{Q} \cdot I_{\lambda}\right] 2^{n-4} \operatorname{dim}(D)=2^{n}\left(2^{n-3}+1\right) 2^{n-4} \operatorname{dim}(D)
$$

with $D$ an irreducible representation of $\mathrm{SU}_{n-4}(2)$. If $D$ is nontrivial, then $\operatorname{dim}(D) \geq\left(2^{n-4}-2\right) / 3$, too large for $n \geq 7$. Thus $\mathrm{SU}_{n-4}(2)$ has a trivial composition factor in the socle of $V_{\lambda}$, and the (unique) faithful representation of $R$ occurs in the socle of $V_{\lambda}$. But then the same is true for $V_{\lambda^{\prime}}$ and we find a trivial $\widetilde{U} \cdot I_{\lambda}$-composition factor in the socle of $X(V)$. Arguing as before we obtain the desired bound.

\section{C. Symplectic groups.}

We next deal with the symplectic groups $\mathrm{S}_{2 n}(q), n \geq 3$ :

Proposition 5.5. Let $G=\mathrm{S}_{2 n}(q)$, q even, $n \geq 3,(n, q) \neq(3,2), \ell \neq 2$, $(q, \ell) \neq(2,3)$, and $V$ a self-dual absolutely irreducible faithful $\mathbb{F} G$-module. Then $X(V)$ is reducible for $X \in\left\{\Lambda^{2}, \mathrm{Sym}^{2}\right\}$ unless possibly if $X=\Lambda^{2}$ and

$$
\frac{\left(q^{n}-1\right)\left(q^{n}-q\right)}{2(q+1)} \leq \operatorname{dim}(V) \leq \begin{cases}2\left(q^{2 n-1}-1\right) & \text { if } q=2, \\ q^{n}\left(q^{n-1}-1\right) & \text { if } q=4, \\ \frac{1}{2}\left(q^{n}-q\right)^{2} /(q-1) & \text { if } q=8\end{cases}
$$

Proof. The case $G=\mathrm{S}_{8}(2)$ can be checked from [12] so we may also assume $(n, q) \neq(4,2)$. Let $G=\mathrm{S}_{2 n}(q)$ and $V$ a self-dual absolutely irreducible faithful $\mathbb{F} G$-module such that $X(V)$ is irreducible. Let $H:=\mathrm{S}_{2}(q) \times \mathrm{S}_{2 n-2}(q) \leq G$ 
be the stabilizer of a 2-dimensional subspace of the natural module. Upon restriction to the Sylow 2-subgroup $U$ of the first factor of $H$ the module $V$ decomposes into a direct $\operatorname{sum} \oplus_{\lambda} V_{\lambda}, \lambda \in \operatorname{Hom}\left(U, \mathbb{F}^{\times}\right)$. The $V_{\lambda}$ for $\lambda \neq 1$ are permuted by the normalizer of $U$ in the first factor of $H$, so they are isomorphic $\mathrm{S}_{2 n-2}(q)$-modules. For $q \geq 4$ we thus obtain at least 3 trivial $\mathrm{S}_{2 n-2}(q)$-composition factors in the socle of $X(V)$. Hence $X(V)$ is a constituent of $1_{\mathrm{S}_{2 n-2}(q)}^{G}$. If $q=2, \ell \neq 3$, we consider instead the eigenspaces $V_{\lambda}$ of the element of order 3 in the first factor $\mathrm{S}_{2}(2)$ of $H$ and reach the same conclusion. But the irreducible complex characters of $\mathrm{S}_{2}(q)$ have degree at most $q+1$, so we obtain the estimate

$$
\operatorname{dim}(X(V)) \leq(q+1)[G: H]=\frac{q^{2 n-2}\left(q^{2 n}-1\right)}{q-1} .
$$

On the other hand by $[\mathbf{2 3}]$ any faithful $\mathbb{F} G$-module $V$ satisfies

$$
\operatorname{dim}(V) \geq \frac{\left(q^{n}-1\right)\left(q^{n}-q\right)}{2(q+1)} .
$$

This leads to a contradiction unless $q \leq 8$.

Next we restrict $V$ to the unipotent radical $U$ of the maximal parabolic subgroup $N$ in Table 3.5, and decompose $V$ as

$$
V=\bigoplus_{\lambda \in \operatorname{Hom}\left(U, \mathbb{F}^{\times}\right)} V_{\lambda}
$$

into $U$-isotypic components. Now $|U|=q^{2 n-1}$, and the Levi factor $L=$ $\mathrm{CSp}_{2 n-2}(q)$ has orbits $\mathrm{O}_{1}, \mathrm{O}_{2}, \mathrm{O}_{3}, \mathrm{O}_{4}$ of lengths

$$
1, q^{2 n-2}-1, \frac{1}{2} q^{n-1}\left(q^{n-1}-1\right)(q-1), \frac{1}{2} q^{n-1}\left(q^{n-1}+1\right)(q-1)
$$

on $\operatorname{Hom}\left(U, \mathbb{F}^{\times}\right)$. All elements in $O_{2}$ have the invariant 1-dimensional subspace of $U$ in their kernel, so since $V$ is faithful, some $V_{\lambda}$ for $\lambda$ in $O_{3}$ or $O_{4}$ has to be nontrivial. Writing $d_{\lambda}$ for its dimension we get

$$
\operatorname{dim}(V) \geq \frac{1}{2} q^{n-1}\left(q^{n-1}-1\right)(q-1) d_{\lambda}
$$

and comparison with (5.6) yields $d_{\lambda}=1$ if $q=8, d_{\lambda} \leq 2$ if $q=4, d_{\lambda} \leq 6$ if $q=2$ and $n \geq 5$, respectively. Thus the stabilizer $I_{\lambda}$ in $L$ of $\lambda$ acts trivially on $V_{\lambda}$. Hence if $X=\mathrm{Sym}^{2}$ or if $d_{\lambda}>1$ then for each $\lambda$ in a fixed orbit the module $X(V)$ has a trivial $I_{\lambda}$-composition factor in the socle. But $L$ permutes these $\lambda$, thus we obtain a trivial constituent in $X(V)$ for the derived group of $L$. This forces $\operatorname{dim}(X(V)) \leq q^{2 n}-1$ which gives a contradiction to the Seitz-Zalesskii bound.

So we have $q \leq 8, d_{\lambda} \leq 1$ for $\lambda \in O_{3} \cup O_{4}$ and $X=\Lambda^{2}$. For $q=4,8$ the upper bound stated in the Proposition now follows from (5.6). For $q=2$ we obtain at least $\operatorname{dim}(V) \leq 2(|U|-1)$. Indeed, each nontrivial orbit can occur at most once, and the dimension of the fixed point space cannot be 
larger than the commutator, for otherwise the parabolic subgroup $N$ and its opposite (which together generate $G$ ) would have a common fixed vector.

Proposition 5.7. Let $G=\mathrm{S}_{2 n}(2), n \geq 4, \ell=3$, and $V$ a self-dual absolutely irreducible faithful $\mathbb{F} G$-module. Then $X(V)$ is reducible for $X \in$ $\left\{\Lambda^{2}, \mathrm{Sym}^{2}\right\}$ unless possibly if

$$
\operatorname{dim}(X(V)) \leq 2^{4 n-8} \frac{\left(2^{2 n}-1\right)\left(2^{2 n-2}-1\right)}{5} .
$$

Proof. Let $H=\mathrm{S}_{4}(2) \times \mathrm{S}_{2 n-4}(2)$ and note that $\mathrm{S}_{4}(2)$ is isomorphic to the symmetric group $\mathfrak{S}_{6}$. We restrict $V$ to a subgroup of order 5 of the first factor. Since elements of order 5 are rational in $\mathfrak{S}_{6}$, the eigenspaces for the nontrivial eigenvalues are permuted transitively and hence are isomorphic as $\mathrm{S}_{2 n-4}(2)$-modules. Thus in $X(V)$ we find a trivial $\mathrm{S}_{2 n-4}(2)$-module in the socle. The largest degree of a 3 -modular irreducible for $\mathfrak{S}_{6}$ is 9 , hence we find an $H$-module of dimension at most 9 in the socle of $X(V)$. This shows

$$
\operatorname{dim}(X(V)) \leq 2^{4 n-4} \frac{\left(2^{2 n}-1\right)\left(2^{2 n-2}-1\right)}{80}
$$

as claimed.

In $\S 7$ we will show that $G=\mathrm{S}_{2 n}(2)$ does in fact lead to examples of irreducible tensor products and irreducible alternating squares.

\section{D. Orthogonal groups.}

The case of orthogonal groups is the least pleasant:

Proposition 5.8. Let $G=\mathrm{O}_{2 n}^{\epsilon}(q), n \geq 4, q$ even, $\ell \neq 2,(q, \ell) \neq(2,3)$, and $V$ a self-dual absolutely irreducible faithful $\mathbb{F} G$-module. Then $X(V)$ is reducible for $X \in\left\{\Lambda^{2}, \mathrm{Sym}^{2}\right\}$ unless possibly if $q=2,4$ and

$$
\operatorname{dim}(X(V)) \leq \begin{cases}\frac{1}{2} q^{2 n-2}\left(q^{n}-\epsilon 1\right)\left(q^{n-1}+\epsilon 1\right) /(q-1) & \text { if } q=4, \ell=5, \\ \frac{1}{2} q^{2 n-2}\left(q^{n}-\epsilon 1\right)\left(q^{n-1}-\epsilon 1\right) /(q+1) & \text { if } q=2 \text { or } \ell \neq 5 .\end{cases}
$$

Proof. Let $G=\mathrm{O}_{2 n}^{\epsilon}(q)$ and let $\delta \in\{ \pm\}$ be such that $\ell \backslash(q-\delta 1)$ and $q-\delta 1>1$ (which is possible since $(q, \ell) \neq(2,3)$ ). We restrict $V$ to a natural subgroup $\mathrm{O}_{2}^{\delta}(q) \times \mathrm{O}_{2 n-2}^{\epsilon \delta}(q)$. Let $V_{\lambda}, V_{\lambda^{-1}}$ denote eigenspaces for the element of order $q-\delta 1$ in the first factor of a nontrivial $(q-\delta 1)$-th root of unity $\lambda$ and its inverse. Since $V$ is self-dual, both $V_{\lambda}, V_{\lambda^{-1}}$ are singular with respect to the nondegenerate form on $V$ and dual to each other. Thus $V_{\lambda} \otimes V_{\lambda^{-1}} \leq X(V)$ has a trivial $\mathrm{O}_{2 n-2}^{\epsilon \delta}(q)$-composition factor in the socle. If $X(V)$ is irreducible this yields the upper bound

$$
\operatorname{dim}(X(V)) \leq \frac{q^{2 n-2}\left(q^{n}-\epsilon 1\right)\left(q^{n-1}+\epsilon 1\right)}{2(q-1)} .
$$


Observe that if $\operatorname{gcd}(\ell, q+1)=1$ then we may choose $\delta=-$, which gives the better bound

$$
\operatorname{dim}(X(V)) \leq \frac{q^{2 n-2}\left(q^{n}-\epsilon 1\right)\left(q^{n-1}-\epsilon 1\right)}{2(q+1)} .
$$

On the other hand, let $P=U . L$ be the parabolic subgroup with Levi complement $L$ of type $\mathrm{O}_{2 n-2}^{\epsilon}(q)$. The restriction of $V$ to the unipotent radical $U$ of $P$ decomposes as $V=\oplus_{\lambda} V_{\lambda}$ for $\lambda \in \operatorname{Hom}\left(U, \mathbb{F}^{\times}\right)$. The Levi factor $L$ acts on $U$ and hence on $\operatorname{Hom}\left(U, \mathbb{F}^{\times}\right)$as on its natural module. It thus has two nontrivial orbits on $\operatorname{Hom}\left(U, \mathbb{F}^{\times}\right)$of lengths $\left(q^{n-1}-\epsilon 1\right)\left(q^{n-2}+\epsilon 1\right)$ and $q^{n-2}\left(q^{n-1}-\epsilon 1\right)(q-1)$, consisting of isotropic respectively anisotropic elements. We first claim that $d_{\lambda}:=\operatorname{dim}\left(V_{\lambda}\right)=0$ for anisotropic $\lambda$ unless possibly $q=4, d_{\lambda}=1$, or $q=2, d_{\lambda} \leq 2$.

So assume that $d_{\lambda} \neq 0$. Then

$$
\operatorname{dim}(V) \geq q^{n-2}\left(q^{n-1}-\epsilon 1\right)(q-1) d_{\lambda} .
$$

Comparison with (5.9) yields $d_{\lambda} \leq 1$ if $q \geq 4$ respectively $d_{\lambda} \leq 3$ if $q=2$. Thus $V_{\lambda}$ has to be the trivial module for the stabilizer $I_{\lambda}=\mathrm{O}_{2 n-3}(q)$ of $\lambda$ in $L$. If $q \geq 4$ the group $I_{\lambda}$ stabilizes at least three different elements of $\operatorname{Hom}\left(U_{1}, \mathbb{F}^{\times}\right)$. Thus if $d_{\lambda}>1$ or $q \geq 4$ we get a trivial $U_{1}^{\prime}: I_{\lambda}$-constituent in the socle of $X(V)$, where $U_{1}^{\prime}=\operatorname{ker}(\lambda)$, hence a linear constituent for $U_{1}: I_{\lambda}$ extended by an element of order $q-1$. So we obtain the better upper bound

$$
\operatorname{dim}(X(V)) \leq \frac{q^{n-2}\left(q^{n}-\epsilon 1\right)\left(q^{2 n-2}-1\right)}{q-1}
$$

which violates (5.11) unless $q=4, d_{\lambda}=1$, or $q=2, d_{\lambda} \leq 2$.

So next assume that $d_{\lambda} \neq 0$ for an isotropic $\lambda$. Then

$$
\operatorname{dim}(V) \geq\left(q^{n-1}-\epsilon 1\right)\left(q^{n-2}+\epsilon 1\right) d_{\lambda},
$$

and comparison with (5.9) shows that $d_{\lambda}<2 q-2$. But the smallest degree of a non-linear representation of $I_{\lambda}$ is at least $2(q-1)$ unless $n=4, q \in\{2,4\}$, $\epsilon=+$. (Note that for $n=4, \epsilon=+$, we have $I_{\lambda}=\mathrm{L}_{2}(q)$ 2 2.) Thus either $G=\mathrm{O}_{8}^{+}(2), G=\mathrm{O}_{8}^{+}(4)$, or $I_{\lambda}^{\prime}$ has to act by a linear character of order at most 2 on $V_{\lambda}$ (in fact, trivially if $(n, \epsilon) \neq(4,+)$ ). So each $X\left(V_{\lambda}\right)$ contains a trivial $I_{\lambda}^{\prime}$-composition factor in the socle. The group $L_{1}^{\prime}$ permutes the isotropic $\lambda$, so we get a trivial constituent for $P^{\prime}$ in $X(V)$. This yields $\operatorname{dim}(X(V)) \leq\left(q^{n}-\epsilon 1\right)\left(q^{n-1}+\epsilon 1\right)$, which is a contradiction to (5.12).

Thus if $X(V)$ is irreducible, then $q=2,4$ and $\operatorname{dim}(X(V))$ is bounded as either in (5.10) or (5.9).

Proposition 5.13. Let $G=\mathrm{O}_{2 n}^{\epsilon}(2), n \geq 5$, $\ell=3$, and $V$ a self-dual absolutely irreducible faithful $\mathbb{F} G$-module. Then $X(V)$ is reducible unless possibly if

$$
\operatorname{dim}(X(V)) \leq 2^{4 n-6}\left(2^{n}-\epsilon 1\right)\left(2^{2 n-2}-1\right)\left(2^{n-2}-\epsilon 1\right) / 15 .
$$


Proof. Let $H=\mathrm{O}_{4}^{-}(2) \times \mathrm{O}_{2 n-4}^{-\epsilon}(2)$. The first factor is isomorphic to $\mathrm{L}_{2}(4)$. The nontrivial eigenspaces of elements of order 5 in this factor yield isomorphic $\mathrm{O}_{2 n-4}^{-\epsilon}(2)$-modules, and hence we force a trivial constituent for $\mathrm{O}_{2 n-4}^{-\epsilon}(2)$ in the socle of $X(V)$. Since the largest 3-modular degree of $\mathrm{L}_{2}(4)$ is 4 the claim follows.

\section{E. Large exceptional groups.}

For the following statement we collect the lower bounds for cross-characteristic representations of certain exceptional groups from Propositions 4.1 and 4.2 respectively from [10].

$$
l(G)= \begin{cases}q\left(q^{4}-q^{2}+1\right)-1 & \text { if } G={ }^{3} D_{4}(q) \\ q\left(q^{4}+1\right)\left(q^{6}+q^{3}+1\right)-1 & \text { if } G=E_{6}(q) \\ q\left(q^{4}+1\right)\left(q^{6}-q^{3}+1\right)-2 & \text { if } G={ }^{2} E_{6}(q) \\ q\left(q^{4}-q^{2}+1\right)\left(q^{12}+q^{10}+q^{8}+q^{6}+q^{4}+q^{2}+1\right)-2 & \text { if } G=E_{7}(q) \\ q\left(q^{6}+1\right)\left(q^{10}+1\right)\left(q^{12}+1\right)-3 & \text { if } G=E_{8}(q) .\end{cases}
$$

Proposition 5.14. Let $G={ }^{3} D_{4}(q), E_{6}(q) s c,{ }^{2} E_{6}(q) s c, E_{7}(q)$ or $E_{8}(q)$, $q=2^{n}>2, \ell \neq 2$, and $V$ a self-dual absolutely irreducible faithful $\mathbb{F} G$ module. Then $X(V)$ is reducible unless possibly if $X=\Lambda^{2}$, and

$$
l(G) \leq \operatorname{dim}(V)<2 l(G) .
$$

Proof. 1) Let $N=Q . L$ be the maximal parabolic subgroup of $G$ from Table 3.5, with special unipotent radical $Q$ of type $q^{1+2 k}$ where $k=4,10,10,16$, 28 respectively. The restriction of $V$ to $Q$ splits into the centralizer $V_{1}:=$ $C_{V}(Q)$, the part $V_{2}$ on which $Q$ acts nontrivial linearly, and $V_{3}:=[Z(Q), V]$. The non-linear characters of $Q$ (of degree $q^{k}$ ) are indexed by the $q-1$ nontrivial linear characters of the center $Z(Q)$. Since $Q$ is normal in $N$, each isotypic part of $\left.V\right|_{Q}$ is an $L^{\prime}$-module.

Let $M$ be an isotypic part of $V_{3}$ of dimension $d q^{k}$. By Lemma 2.3 and Proposition 2.4 in [19] the tensor square of $M$ contains an $N$-submodule of dimension $d^{2}$. Since $V$ is self-dual and $2 \mid q$ we deduce that $X(V)$ also contains an $N$-submodule of dimension $d(d \pm 1) / 2$ (the sign depending on $X$ and the type of $Q$ ). If $d>1$, this gives the upper bound $\operatorname{dim}(X(V)) \leq[G$ : $N] d(d \pm 1) / 2$, while on the other hand clearly $\operatorname{dim}(V) \geq d q^{k}(q-1)$. Using the values

$$
[G: N]= \begin{cases}\frac{\left(q^{6}-1\right)\left(q^{4}-q^{2}+1\right)}{q-1} & \text { if } G={ }^{3} D_{4}(q), \\ \frac{\left(q^{4}+1\right)\left(q^{6}+q^{3}+1\right)\left(q^{12}-1\right)}{q-1} & \text { if } G=E_{6}(q), \\ \frac{\left(q^{4}+1\right)\left(q^{6}-q^{3}+1\right)\left(q^{12}-1\right)}{q-1} & \text { if } G={ }^{2} E_{6}(q), \\ \frac{\left(q^{4}+q^{2}+1\right)\left(q^{14}-1\right)\left(q^{18}-1\right)}{(q-1)\left(q^{2}-1\right)} & \text { if } G=E_{7}(q), \\ \frac{\left(q^{10}+1\right)\left(q^{24}-1\right)\left(q^{30}-1\right)}{(q-1)\left(q^{6}-1\right)} & \text { if } G=E_{8}(q),\end{cases}
$$


this leads to a contradiction for $q \geq 4$. Thus all $Q$-isotypic parts of $V_{3}$ are irreducible, and $\operatorname{dim}\left(V_{3}\right)=q^{k}(q-1)$.

If $Z^{\prime}$ is a subgroup of index 2 in $Z(Q)$, then $Q / Z^{\prime}$ is an extraspecial 2group of type $\epsilon$ for some $\epsilon= \pm$. Then any non-linear irreducible character of $Q$ has Schur-Frobenius indicator $\epsilon 1$. Since $d=1$ and since we are in the bad case, we see that $(\epsilon, X)=\left(+, \Lambda^{2}\right)$ or $\left(-, \mathrm{Sym}^{2}\right)$.

We next estimate the dimension of $V_{2}$. Let $\mu$ denote the Brauer character of $V$ and $\mu_{i}$ the Brauer character of $V_{i}, i=2,3$. Let $g \in Z(Q)$ be a central involution of $Q$. Since $V_{3}$ contains all non-linear characters of $Q$ exactly once, $\mu_{3}(g)=-q^{k}$ and $\mu(g)=\operatorname{dim}(V)-\operatorname{dim}\left(V_{3}\right)-q^{k}=\operatorname{dim}(V)-q^{k+1}$ since $Z(Q)$ acts trivially on $V_{1} \oplus V_{2}$. We now use the main result of [3] which states that the value of any nontrivial irreducible Brauer character on any non-identity unipotent element is equal to at most $3 / 4$ of its degree. Plugging this into our above computations we get

$$
\operatorname{dim}(V) \leq 4 q^{k+1} .
$$

2) Let first $G \neq{ }^{3} D_{4}(q), E_{6}(q)$. The linear characters of $Q$ are just the irreducible characters of $U:=Q / Z(Q)$. The orbits of $L$ on $\operatorname{Hom}\left(U, \mathbb{F}^{\times}\right)$are known for $G=E_{7}(q), E_{8}(q)[\mathbf{1 0}]$ and given in the proof of Proposition 4.2 for ${ }^{2} E_{6}(q)$.

Comparison with the upper bound $\operatorname{dim}\left(V_{2}\right) \leq 3 q^{k+1}+q^{k}$ shows that in all cases only the shortest nontrivial orbit $O_{1}$ can occur. For $\lambda \in O_{1}$ let $V_{\lambda}$ denote the $\lambda$-isotypic component of $V_{2}$ and $I_{\lambda}$ the stabilizer of $\lambda$ in $L$ (of semisimple type $\left.\mathrm{SL}_{3}\left(q^{2}\right), \mathrm{SL}_{6}(q), E_{6}(q)\right)$. The lower bounds for nontrivial representations of $I_{\lambda}^{\prime}$ compared with the upper bound for $\operatorname{dim}\left(V_{2}\right)$ above implies that $I_{\lambda}^{\prime}$ acts trivially on $V_{\lambda}$. Assume that $d_{\lambda}:=\operatorname{dim}\left(V_{\lambda}\right) \geq 2$. Then $X\left(V_{\lambda}\right)$ contains a trivial composition factor for $I_{\lambda}^{\prime}$, but clearly also for $U$ (since $2 \mid q$ ). The representation of $L^{\prime}$ on the $L^{\prime}$-orbit of that trivial submodule is the permutation module of $L^{\prime}$ on the cosets of $I_{\lambda}^{\prime}$, thus it contains a trivial $L^{\prime}$-composition factor. We hence obtain that $\operatorname{dim}(X(V)) \leq\left[G: N^{\prime}\right]$ which gives a contradiction to the lower bound for $\operatorname{dim}(V)$ from $[23]$. Thus $d_{\lambda} \leq 1$ and we find

$$
\operatorname{dim}(V) \leq 2\left(q^{k}(q-1)+\left|O_{1}\right|\right)<2 l(G) .
$$

3) Let now $G={ }^{3} D_{4}(q)$. We first claim that $G$ is generated by four long root elements. Indeed, the normalizer of a Coxeter torus is maximal in $G$, and does not contain long root elements. Thus any four long root elements whose product is a generator of a Coxeter torus must generate $G$. Using the character table in [2] it can be verified that the structure constant for the corresponding 5 -tuples is nonzero.

Thus in any irreducible representation $V$ the \pm 1 -eigenspaces of a long root element $g$ can have dimension at most $3 / 4 \operatorname{dim}(V)$. If $\mu$ denotes the corresponding Brauer character, it follows that $|\mu(g) / \mu(1)| \leq 1 / 2$. This yields $\operatorname{dim}(V) \leq 2 q^{5}$. Thus again only the shortest orbit $O_{1}$ of $L$ on the 
nontrivial linear characters of $Q / Z(Q)$ can occur. In this case the stabilizer $I_{\lambda}$ of $\lambda \in O_{1}$ is a subgroup of order $q^{3}\left(q^{2}+q+1\right)$ of the Borel subgroup. Its non-linear irreducible characters have degree $q^{2}+q+1$. Comparison with the upper bound for $\operatorname{dim}(V)$ shows that $I_{\lambda}$ has to act linearly. Since $V$ is self-dual, $V_{\lambda}$ is also self-dual. If $\operatorname{dim}\left(V_{\lambda}\right)>1$ then $X(V)$ contains a trivial composition factor for $I_{\lambda}$. Arguing as in 2) above this leads to a contradiction, forcing $d_{\lambda} \leq 1$.

4) Let $G=E_{6}(q)$. Let $P=U \cdot \operatorname{Spin}_{10}^{+}(q) \cdot Z_{q-1}$ be the $D_{5}$-parabolic subgroup of $G$. Its unipotent radical $U$ is elementary abelian of order $q^{16}$, and the Levi factor $L$ has two nontrivial orbits $O_{1}, O_{2}$ on $\operatorname{Hom}\left(U, \mathbb{F}^{\times}\right)$, of lengths

$$
\left(q^{3}+1\right)\left(q^{8}-1\right), \quad q^{3}\left(q^{5}-1\right)\left(q^{8}-1\right)
$$

(see [10]). For $\lambda \in \operatorname{Hom}\left(U, \mathbb{F}^{\times}\right)$let $V_{\lambda}$ denote the $\lambda$-isotypic component of the restriction of $V$ to $U$ and $d_{\lambda}$ its dimension. By part 1), $d_{\lambda}=0$ if $\lambda \in O_{2}$ and $d_{\lambda} \leq 5$ if $\lambda \in O_{1}$. Let $\lambda \in O_{1}$ and denote by $I_{\lambda}$ the stabilizer of $\lambda$ in $L_{2}$, with semisimple part $\mathrm{SL}_{5}(q)$. Since any nontrivial representation of $I_{\lambda}^{\prime}$ has dimension at least $\left(q^{5}-1\right) /(q-1)-5$ by $[23], I_{\lambda}^{\prime}$ has to act trivially on $V_{\lambda}$. Assume that $d_{\lambda} \geq 2$. Then again $X\left(V_{\lambda}\right)$ contains a trivial composition factor for $I_{\lambda}^{\prime}$. We may proceed as before to obtain that $\operatorname{dim}(X(V)) \leq\left[G: P^{\prime}\right]$, again contradicting the lower bound for $\operatorname{dim}(V)$ from [13]. Thus $d_{\lambda}=1$ for $\lambda \in O_{1}$. In particular we find

$$
\operatorname{dim}(V) \leq 2\left|O_{1}\right|=2\left(q^{3}+1\right)\left(q^{8}-1\right)<2 l(G)
$$

as claimed.

5) Finally, observe that $(\epsilon, X)=\left(+, \Lambda^{2}\right)$. For, $\left.V\right|_{Q}$ contains linear characters of $Q$ with multiplicity 1 , and clearly those characters are of type + , whence $V$ itself is of type + .

Proposition 5.15. Let $G={ }^{2} F_{4}\left(q^{2}\right)$ with $q^{2}>2$, or $G=F_{4}(q)$ with $2 \neq q$ even, and let $\ell \neq 2$, and $V$ a self-dual absolutely irreducible faithful $\mathbb{F} G$ module. Then $X(V)$ is reducible unless possibly if

$$
\operatorname{dim}(V) \leq 2 q^{11}(q+1) .
$$

Proof. The largest degree of an irreducible complex character of $G$ is $\left(q^{2}-\right.$ 1) $\left(q^{6}+1\right)\left(q^{8}-1\right)\left(q^{12}+1\right) /\left(q^{2}-\sqrt{2} q+1\right)^{2}$, respectively $\left(q^{2}-1\right)\left(q^{6}-1\right)\left(q^{8}-\right.$ 1) $\left(q^{12}-1\right) /(q-1)^{4}$, thus we get the trivial upper bound $\operatorname{dim}(V) \leq 2 q^{11}(q+$ $1)$.

\section{The sporadic groups.}

Proposition 6.1. Let $G$ be a covering group of a sporadic simple group in $\left\{\mathrm{M}_{11}, \mathrm{M}_{12}, \mathrm{~J}_{1}, \mathrm{M}_{22}, \mathrm{~J}_{2}, \mathrm{M}_{23}, \mathrm{HS}, \mathrm{J}_{3}, \mathrm{M}_{24}, \mathrm{McL}, \mathrm{He}, \mathrm{Suz}, \mathrm{Co}_{3}, \mathrm{Co}_{2}\right\}$, $\ell \geq 0$ and $V$ an absolutely irreducible faithful $\mathbb{F} G$-module. Then $X(V)$ is reducible for $X \in\left\{\widetilde{\Lambda}^{2}, \widetilde{\Sigma}^{2}, \widetilde{A}\right\}$ unless $(G, \ell, \operatorname{dim}(V), X)$ are as in Table 6.2. 
Table 6.2. Small sporadic groups.

\begin{tabular}{|c|c|c|c|c|c|}
\hline$G$ & $\ell$ & $\operatorname{dim}(V)$ & $\widetilde{\Lambda}^{2}$ & $\widetilde{\Sigma}^{2}$ & $\widetilde{A}$ \\
\hline $\mathrm{M}_{11}$ & 3 & 5 & & & $x$ \\
\hline $\mathrm{M}_{11}$ & 11 & 9 & & $x$ & \\
\hline $\mathrm{M}_{11}$ & $\neq 11$ & 10 & $x$ & & \\
\hline $\mathrm{M}_{11}$ & $\neq 2,3$ & 11 & $x$ & & \\
\hline 2. $\mathrm{M}_{12}$ & 3 & 6 & & & $x$ \\
\hline $\mathrm{M}_{12}$ & 2 & 10 & $\times$ & & \\
\hline $\mathrm{M}_{12}$ & 3 & 10 & $x$ & $\times$ & \\
\hline 2. $\mathrm{M}_{12}$ & $\neq 2$ & 10 & & & $x$ \\
\hline $\mathrm{M}_{12}$ & $\neq 2,3$ & 11 & $x$ & & \\
\hline 2. $\mathrm{M}_{12}$ & $\neq 2,3$ & 12 & $x$ & & \\
\hline $\mathrm{J}_{1}$ & 11 & 7 & & $x$ & \\
\hline $3 . \mathrm{M}_{22}$ & 2 & 6 & & & $x$ \\
\hline $\mathrm{M}_{22}$ & 2 & 10 & & & $x$ \\
\hline 2. $\mathrm{M}_{22}$ & 7 & 10 & $x$ & $x$ & \\
\hline 2. $\mathrm{M}_{22}$ & $\neq 2,7$ & 10 & & & $x$ \\
\hline $\mathrm{M}_{22}$ & 11 & 20 & $x$ & & \\
\hline $\mathrm{M}_{22}$ & $\neq 2,11$ & 21 & $x$ & & \\
\hline $\mathrm{J}_{2}$ & 2 & 6 & $x$ & & \\
\hline $2 . \mathrm{J}_{2}$ & $\neq 2$ & 6 & $x$ & $\times$ & \\
\hline $\mathrm{J}_{2}$ & 3 & 13 & & $\times$ & \\
\hline $2 . \mathrm{J}_{2}$ & $\neq 2$ & 14 & $x$ & & \\
\hline $\mathrm{M}_{23}$ & 2 & 11 & & & $\times$ \\
\hline $\mathrm{M}_{23}$ & 23 & 21 & $x$ & $x$ & \\
\hline $\mathrm{M}_{23}$ & $\neq 2,23$ & 22 & $x$ & & \\
\hline $\mathrm{M}_{23}$ & 7 & 45 & $x$ & $\times$ & \\
\hline $\mathrm{M}_{23}$ & $\neq 2,3,5,7$ & 45 & & & $x$ \\
\hline
\end{tabular}

Proof. This can be checked from the known tables of Brauer characters $[12]$. 
Table 6.2. Small sporadic groups (continued).

\begin{tabular}{|c|c|c|c|c|c|}
\hline$G$ & $\ell$ & $\operatorname{dim}(V)$ & $\widetilde{\Lambda}^{2}$ & $\widetilde{\Sigma}^{2}$ & $\widetilde{A}$ \\
\hline HS & 5 & 21 & $x$ & & \\
\hline HS & $\neq 2,5$ & 22 & $x$ & & \\
\hline $3 . \mathrm{J}_{3}$ & 2 & 9 & & & $x$ \\
\hline $\mathrm{J}_{3}$ & 3 & 18 & $\times$ & & \\
\hline $3 . \mathrm{J}_{3}$ & $\neq 3$ & 18 & & & $x$ \\
\hline $\mathrm{M}_{24}$ & 2 & 11 & & & $x$ \\
\hline $\mathrm{M}_{24}$ & 3 & 22 & $\times$ & $\times$ & \\
\hline $\mathrm{M}_{24}$ & $\neq 2,3$ & 23 & $x$ & & \\
\hline $\mathrm{M}_{24}$ & 7 & 45 & $\times$ & $\times$ & \\
\hline $\mathrm{M}_{24}$ & $\neq 2,3,7$ & 45 & & & $x$ \\
\hline $\mathrm{McL}$ & 3 & 21 & $\times$ & & \\
\hline $\mathrm{McL}$ & 5 & 21 & $\times$ & $\times$ & \\
\hline $\mathrm{McL}$ & 2 & 22 & $\times$ & & \\
\hline $\mathrm{McL}$ & $\neq 2,3,5$ & 22 & $\times$ & $x$ & \\
\hline $\mathrm{Ru}$ & 2 & 28 & $\times$ & & \\
\hline $2 . \mathrm{Ru}$ & $\neq 2$ & 28 & & & $x$ \\
\hline 3.Suz & 2 & 12 & & & $x$ \\
\hline 2.Suz & 3 & 12 & $\times$ & $x$ & \\
\hline 6.Suz & $\neq 2,3$ & 12 & & & $\times$ \\
\hline $\mathrm{Co}_{3}$ & 2,3 & 22 & $\times$ & & \\
\hline $\mathrm{Co}_{3}$ & $\neq 2,3$ & 23 & $x$ & $x$ & \\
\hline $\mathrm{Co}_{3}$ & 5 & 230 & $\times$ & & \\
\hline $\mathrm{Co}_{3}$ & $\neq 2,3,5$ & 253 & $\times$ & & \\
\hline $\mathrm{Co}_{2}$ & 2 & 22 & $\times$ & & \\
\hline $\mathrm{Co}_{2}$ & $\neq 2$ & 23 & $\times$ & $x$ & \\
\hline
\end{tabular}

Complete results for sporadic groups in characteristic 0 are given in the next section. 


\section{Results in characteristic 0 .}

In this section we complete the answer in the case of characteristic 0 (or more generally, in the case of coprime characteristic). In order to achieve this, by the remark following the proof of Theorem 3.1, we have to consider those groups in the bad case not handled in Section 5. Here our proof relies very much on Lusztig's classification of the (degrees of) irreducible characters of groups of Lie type, more precisely, on the Jordan decomposition of characters and the determination of the degrees of unipotent characters. We refer to [25] for a short survey of the phenomenology of the necessary results.

\section{A. The classical groups in characteristic $\ell=0$.}

First we handle the unitary groups.

Proposition 7.1. Let $G$ be a cover of $\mathrm{U}_{n}(q)$ with $n \geq 4$ and $V$ a nontrivial irreducible $\mathbb{C} G$-module. Then $X(V)$ is reducible unless $q=2,3$ and $V$ is a Weil module of $G$.

Proof. The results of $\S \S 3,5$ allow us to assume that we are in the bad case and that $q=2$. The cases $n \leq 6$ can be checked directly, hence we assume that $n \geq 7, V$ is a module for $G=\mathrm{SU}_{n}(2), V$ is not a Weil module, and $X(V)$ is irreducible.

Suppose that $V$ is extendible to $H:=\mathrm{GU}_{n}(2)$. But $V$ is self-dual, so $V^{H}$ is the sum of three irreducible $H$-modules, and at least one of them, which we denote by the same letter $V$, is self-dual. Consider a pseudoreflection $g$ of order 3 in $H$. Then the nontrivial eigenspaces of $g$ in $V$ are dual to each other as $C$-modules, where $C=\mathrm{GU}_{1}(2) \times \mathrm{GU}_{n-1}(2)$. Thus $X(V)$ contains the trivial $C$-module, and so $\operatorname{dim}(X(V)) \leq(H: C)=\left(2^{n}-(-1)^{n}\right) 2^{n-1} / 3$, which implies by [25] that $V$ is a Weil module. Consequently, $V$ cannot be extended to $H$. In particular, we are done if $\operatorname{gcd}(n, 3)=1$.

So we may assume that $n \geq 9$. The bound in Proposition 5.3 implies that $\operatorname{dim}(V)<2^{n-1}\left(2^{n-1}+1\right) / 3$. Carefully following the proof of Theorem 4.1 of [25], one can show that $G$ has exactly 9 nontrivial irreducible modules satisfying this bound; namely three Weil modules, one of dimension $\left(2^{n}-\right.$ $\left.(-1)^{n}\right)\left(2^{n-1}+4(-1)^{n}\right) / 9$, two of dimension $\left(2^{n}-(-1)^{n}\right)\left(2^{n-1}-2(-1)^{n}\right) / 9$, and three in the dimension $\left(2^{n}-(-1)^{n}\right)\left(2^{n-1}+(-1)^{n}\right) / 9$. From Lusztig's parametrization of irreducible characters of $G$ and $H$, it follows that all these modules extend to $H$, which completes the proof.

The irreducible $X(V)$ for complex Weil modules $V$ of $\mathrm{SU}_{n}(2)$ and $\mathrm{SU}_{n}(3)$ are determined in $[\mathbf{2 0}]$ and $[\mathbf{1 4}]$.

To handle the symplectic and orthogonal groups, we need the following observation, which follows from Lusztig's classification of unipotent characters.

Lemma 7.2. Let $\chi$ be a complex irreducible unipotent character of a finite group of Lie type $G$ in characteristic $p$. 
(i) Let $G$ be $\mathrm{GL}_{n}(q)$ or $\mathrm{GU}_{n}(q)$. Then the p-part $\chi(1)_{p}$ of $\chi(1)$ is a power of $q$, and this $p$-part is 1 if and only if $\chi$ is trivial.

(ii) Let $G=\mathrm{S}_{2 n}(q)$ and $p=2$. Then either $\chi$ is trivial, or $\chi$ is labeled by one of the symbols

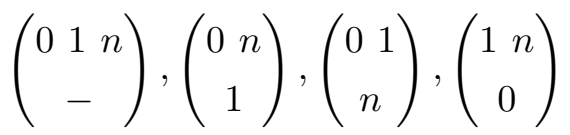

and $\chi(1)_{2}=q / 2$, or $\chi(1)_{2} \geq q$.

(iii) Let $G=\mathrm{O}_{2 n}^{\epsilon}(q)$ with $p=2$ and $n \geq 4$. Then either $\chi$ is trivial, or $\chi$ is the smallest unipotent character of degree $\left(q^{n}-\epsilon\right)\left(q^{n-1}+\epsilon q\right) /\left(q^{2}-1\right)$, or $\chi(1)_{2} \geq q^{2} / 2$.

Proof. We refer to [25], for example, for explicit formulae giving the degree polynomials of unipotent characters. In Case (i), assume $\chi$ is labeled by the partition $\left(\alpha_{1}, \alpha_{2}, \ldots, \alpha_{m}\right)$ of $n$ (with $\left.1 \leq \alpha_{1} \leq \alpha_{2} \leq \ldots \leq \alpha_{m}\right)$ and let $\lambda_{i}=\alpha_{i}+i-1 \geq i$. Then $\chi(1)_{p}$ is $q^{d}$, where

$$
d=\sum_{i=1}^{m-1}(m-i) \lambda_{i}-\sum_{i=2}^{m-1}\left(\begin{array}{l}
i \\
2
\end{array}\right) \geq \sum_{i=1}^{m-1}(m-i) i-\sum_{i=2}^{m-1}\left(\begin{array}{l}
i \\
2
\end{array}\right)=\left(\begin{array}{c}
m \\
2
\end{array}\right) .
$$

In particular, if $d=0$ then $m=1$ and $\chi$ is the trivial character.

In Cases (ii), (iii), unipotent characters $\chi$ are labeled by symbols

$$
\left(\begin{array}{l}
\lambda \\
\mu
\end{array}\right)=\left(\begin{array}{llll}
\lambda_{1} & \lambda_{2} & \ldots & \lambda_{a} \\
\mu_{1} & \mu_{2} & \cdots & \mu_{b}
\end{array}\right)
$$

of rank $n$, where $0 \leq \lambda_{1}<\lambda_{2}<\ldots<\lambda_{a}, 0 \leq \mu_{1}<\mu_{2}<\ldots<\mu_{b},\left(\lambda_{1}, \mu_{1}\right) \neq$ $(0,0)$, with $a-b$ odd in Case (ii) and even in (iii). While estimating the 2-powers dividing $\chi(1)$, we will also occasionally change the rank of the symbols.

Assume $a, b \geq 1$ and $(a, b) \neq(1,1)$. Then we can consider the unipotent character $\chi^{\prime}$ corresponding to

$$
\left(\begin{array}{llll}
\lambda_{1} & \lambda_{2} & \ldots & \lambda_{a-1} \\
\mu_{1} & \mu_{2} & \ldots & \mu_{b-1}
\end{array}\right)
$$

It follows from the explicit degree formulae that $\chi(1)_{2} / \chi^{\prime}(1)_{2}$ is at least $q^{d} / 2$, where

$$
d=\sum_{i=1}^{a-1} \lambda_{i}+\sum_{j=1}^{b-1} \mu_{j}+\sum_{j=1}^{b} \min \left(\lambda_{a}, \mu_{j}\right)+\sum_{i=1}^{a-1} \min \left(\lambda_{i}, \mu_{b}\right)-\left(\begin{array}{c}
a+b-2 \\
2
\end{array}\right),
$$

and $d$ will attain its smallest value when

$$
\left(\begin{array}{l}
\lambda \\
\mu
\end{array}\right)=\left(\begin{array}{cccc}
0 & 1 & \ldots & a-1 \\
1 & 2 & \ldots & b
\end{array}\right),\left(\begin{array}{cccc}
1 & 2 & \ldots & a \\
0 & 1 & \ldots & b-1
\end{array}\right)
$$


for which $d$ is exactly $a+b-2$. It follows that $\chi(1)_{2} \geq q^{a+b-2} / 2$. In Case (ii) we get $\chi(1)_{2} \geq q / 2$. Moreover, if $\chi(1)_{2}=q / 2$ then $a+b=3$, i.e., $(a, b)=(2,1)$. Direct calculation then shows that $\chi$ is labeled by one of the symbols given in the statement.

In Case (iii) $a+b$ is even, so $a+b \geq 4$ and $\chi(1)_{2} \geq q^{2} / 2$. If $(a, b)=(1,1)$, then $G=\mathrm{O}_{2 n}^{+}(q)$. Here either $\chi$ is trivial, or $\left(\begin{array}{l}\lambda \\ \mu\end{array}\right)=\left(\begin{array}{c}1 \\ n-1\end{array}\right)$ and $\chi(1)=$ $\left(q^{n}-1\right)\left(q^{n-1}+q\right) /\left(q^{2}-1\right)$, or $\chi(1)_{2} \geq q^{2} / 2$.

Suppose $b=0$ and $a \geq 3$. Then we can consider the unipotent character

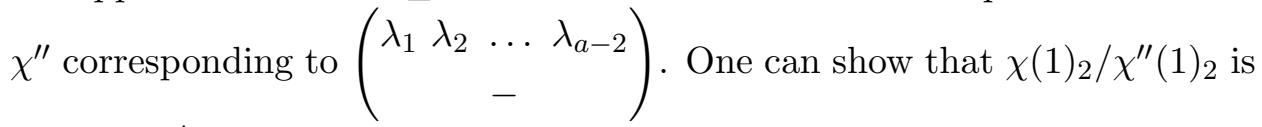
at least $q^{d^{\prime}} / 2$, where

$$
d^{\prime}=2 \sum_{i=1}^{a-2} \lambda_{i}+\lambda_{a-1}-\left(\begin{array}{c}
a-2 \\
2
\end{array}\right)
$$

and $d^{\prime}$ will attain its smallest value when $\lambda=\left(0,1, \ldots, a-2, \lambda_{a}\right)$, for which $d$ is exactly $(a-1)(a-2) / 2$. It follows that $\chi(1)_{2} \geq q / 2$. Moreover, if $\chi(1)_{2}=q / 2$ then $a=3, G=\mathrm{S}_{2 n}(q)$, and $\lambda=(0,1, n)$. In Case (iii) $a$ is even, so $a \geq 4$ and $\chi(1)_{2} \geq q^{3} / 2$.

Finally, let $b=0$ and $a \leq 2$. If $a=2$, then $G=\mathrm{O}_{2 n}^{-}(q)$, and either $\chi$ is trivial, or $\lambda=(1, n-1)$ and $\chi(1)=\left(q^{n}+1\right)\left(q^{n-1}-q\right) /\left(q^{2}-1\right)$, or $\chi(1)_{2} \geq q^{2}$. If $a=1$, then $G=\mathrm{S}_{2 n}(q)$ and $\chi$ is trivial.

Next we handle the bad case for symplectic groups $G=\mathrm{S}_{2 n}(q), q$ even. Let $W=\mathbb{F}_{q}^{2 n}$ be the natural module for $G$, and we consider the permutation character $\omega$ of $G$ on $W$. Then $\omega(g)=q^{\operatorname{dim} \operatorname{Ker}(g-1)}$ for any $g \in G$. We will also consider the class function

$$
\widetilde{\omega}_{n}: g \mapsto(-q)^{\operatorname{dim} \operatorname{Ker}(g-1)} .
$$

Using certain dual pairs in characteristic 2, it was shown in [24] that the permutation action of $G$ on the 1 -spaces of $W$ affords the character $1_{G}+\rho_{1}+$ $\rho_{2}$ where $\rho_{1}$ and $\rho_{2}$ are irreducible characters of degree $\left(q^{n}+1\right)\left(q^{n}-q\right) / 2(q-$ $1)$ and $\left(q^{n}-1\right)\left(q^{n}+q\right) / 2(q-1)$ respectively, and that $\widetilde{\omega}_{n}$ is actually the restriction of the (reducible) Weil character $\sum_{i=0}^{q} \zeta_{n}^{i}$ of $\operatorname{SU}_{2 n}(q)$ to $G$, when $G$ is naturally embedded in $\mathrm{SU}_{2 n}(q)$. Moreover, $\left.\zeta_{n}^{0}\right|_{G}=\alpha_{n}+\beta_{n}$, where $\alpha_{n}$ and $\beta_{n}$ are irreducible characters of degree $\left(q^{n}+1\right)\left(q^{n}+q\right) / 2(q+1)$ and $\left(q^{n}-1\right)\left(q^{n}-q\right) / 2(q+1)$ respectively, and $\left.\zeta_{n}^{i}\right|_{G}=\left.\zeta_{n}^{q+1-i}\right|_{G}=\gamma_{n}^{i}$ is an irreducible character of degree $\left(q^{2 n}-1\right) /(q+1)$ when $1 \leq i \leq q / 2$. If $q=2$, then we will use the notation $\gamma_{n}$ instead of $\gamma_{n}^{1}$. If $\xi$, resp. $\delta$, is a primitive 
$(q+1)^{\text {th }}$-root of unity in $\mathbb{C}$, resp. in $\mathbb{F}_{q^{2}}$, then

$$
\zeta_{n}^{j}(g)=\frac{1}{q+1} \sum_{i=0}^{q} \xi^{i j}(-q)^{\operatorname{dim} \operatorname{Ker}\left(g-\delta^{i}\right)} .
$$

Proposition 7.4. Let $G=\mathrm{S}_{2 n}(2), n \geq 3$. Then the characters $\Lambda^{2}\left(\alpha_{n}\right)$, $\Lambda^{2}\left(\beta_{n}\right), \Lambda^{2}\left(\gamma_{n}\right)$, and $\alpha_{n} \beta_{n}$ are irreducible. Moreover, if $n \geq 4$ then all the other $X(\chi)$ with $\chi \in\left\{\alpha_{n}, \beta_{n}, \gamma_{n}\right\}$ and $X \in\left\{\widetilde{A}, \widetilde{\Sigma}^{2}, \widetilde{\Lambda}^{2}\right\}$, and $\alpha_{n} \gamma_{n}, \beta_{n} \gamma_{n}$, are reducible.

Proof. The cases $n=3,4$ are easy to check, so we will assume $n \geq 5$. We begin with the obvious observation that $\omega^{2}=\widetilde{\omega}_{n}^{2}$. Next, $\mu:=\omega-1_{G}=1_{G}+$ $\rho_{1}+\rho_{2}$ is the permutation character of $G$ on $W^{\times}$. Hence, $\left(\left(\omega-1_{G}\right)^{2}, \omega-1_{G}\right)_{G}$, resp. $\left(\left(\omega-1_{G}\right)^{2},\left(\omega-1_{G}\right)^{2}\right)_{G}$ is the number of $G$-orbits on $W^{\times} \times W^{\times} \times W^{\times}$, resp. on $W^{\times} \times W^{\times} \times W^{\times} \times W^{\times}$, which is 17 , resp. 179 , as can be seen by direct counting. Thus

$$
\begin{gathered}
\left(\widetilde{\omega}_{n}^{2}-3 \cdot 1_{G}-2 \rho_{1}-2 \rho_{2}, \mu\right)_{G}=17, \\
\left(\widetilde{\omega}_{n}^{2}-3 \cdot 1_{G}-2 \rho_{1}-2 \rho_{2}, \widetilde{\omega}_{n}^{2}-3 \cdot 1_{G}-2 \rho_{1}-2 \rho_{2}\right)_{G}=179 .
\end{gathered}
$$

It is known that $\zeta_{n}^{0}$ has Schur-Frobenius indicator 1 , and so do $\alpha_{n}$ and $\beta_{n}$. We write

$$
\begin{array}{ll}
\operatorname{Sym}^{2}\left(\alpha_{n}\right)=1_{G}+\chi_{1}+a_{1} \rho_{1}+b_{1} \rho_{2}, & \Lambda^{2}\left(\alpha_{n}\right)=\chi_{2}+a_{2} \rho_{1}+b_{2} \rho_{2}, \\
\operatorname{Sym}^{2}\left(\beta_{n}\right)=1_{G}+\chi_{3}+a_{3} \rho_{1}+b_{3} \rho_{2}, & \Lambda^{2}\left(\beta_{n}\right)=\chi_{4}+a_{4} \rho_{1}+b_{4} \rho_{2},
\end{array}
$$

$\operatorname{Sym}^{2}\left(\gamma_{n}\right)=c_{1} \cdot 1_{G}+\chi_{5}+a_{5} \rho_{1}+b_{5} \rho_{2}, \quad \Lambda^{2}\left(\gamma_{n}\right)=c_{2} \cdot 1_{G}+\chi_{6}+a_{6} \rho_{1}+b_{6} \rho_{2}$, $\alpha_{n} \beta_{n}=\chi_{7}+a_{7} \rho_{1}+b_{7} \rho_{2}, \alpha_{n} \gamma_{n}=\chi_{8}+a_{8} \rho_{1}+b_{8} \rho_{2}, \beta_{n} \gamma_{n}=\chi_{9}+a_{9} \rho_{1}+b_{9} \rho_{2}$ for some non-negative integers $a_{i}, b_{i}, c_{i}$; furthermore, each $\chi_{i}$ is either 0 or a $G$-character not involving $1_{G}, \rho_{1}, \rho_{2}$. Clearly $c_{1}+c_{2}=1$. From the decomposition

$$
\widetilde{\omega}_{n}^{2}=\alpha_{n}^{2}+\beta_{n}^{2}+4 \gamma_{n}^{2}+2 \alpha_{n} \beta_{n}+4 \alpha_{n} \gamma_{n}+4 \beta_{n} \gamma_{n}
$$

and (7.5) it follows that $a+b=18$, where $a=\sum_{i=0}^{9} t_{i} a_{i}, b=\sum_{i=0}^{9} t_{i} b_{i}$, with $t_{i}=1$ for $1 \leq i \leq 4,2$ for $i=7$, and 4 otherwise. In this case $(a-2)^{2}+(b-2)^{2} \geq 98$. Together with (7.6), this implies that

$$
\left(\sum_{i=1}^{9} t_{i} \chi_{i}, \sum_{i=1}^{9} t_{i} \chi_{i}\right)_{G} \leq 179-9-98=72=\sum_{i=1}^{9} t_{i}^{2} .
$$

On the other hand, by looking at the character degree and using the equality $a+b=18$ we see that all $\chi_{i}$ are nonzero. Thus (7.7) means that the $\chi_{i}$, $1 \leq i \leq 9$, are distinct irreducible characters of $G$.

Next we restrict various characters to the first parabolic subgroup $P$ of $G$. Recall that $P=U . L$, where $U$ is elementary abelian of order $2^{2 n-1}$ and $L \simeq \mathrm{S}_{2 n-2}(2)$. We can define the characters $\alpha_{n-1}, \beta_{n-1}, \gamma_{n-1}$ of $L$ in a 
similar manner. Also, $L$ acts on the set of linear characters of $U$ with four orbits $O_{j}, 1 \leq j \leq 4$, see the proof of Prop. 5.5. Restricting to $L$ and using the explicit formula (7.3), we see that the restriction of $\alpha_{n}, \beta_{n}$, and $\gamma_{n}$ to $L$ involves only the characters $\alpha_{n-1}, \beta_{n-1}, \gamma_{n-1}$. Knowing this information and the length of each $O_{j}$, we can show that

$$
\left.\beta_{n}\right|_{P}=\beta^{\prime}+\beta_{n-1},
$$

where $\beta_{n-1}$ is inflated from $L$ to $P$ and $\left.\beta^{\prime}\right|_{U}=\sum_{\lambda \in O_{3}} \lambda$, in particular, $\beta^{\prime}$ is irreducible over $P$. Thus

$$
2=\left(\left.\beta_{n}\right|_{P},\left.\beta_{n}\right|_{P}\right)_{P}=\left(\beta_{n}^{2}, 1_{P}^{G}\right)_{G}=\left(\beta_{n}^{2}, \mu\right)_{G},
$$

i.e., $a_{3}+b_{3}+a_{4}+b_{4}=1$. By Theorem $3.1 \operatorname{Sym}^{2}\left(\beta_{n}\right)-1_{G}$ is reducible. Consequently, $a_{3}+b_{3}=1, a_{4}=b_{4}=0$, i.e., $\Lambda^{2}\left(\beta_{n}\right)$ is irreducible.

Similarly,

$$
\left.\alpha_{n}\right|_{P}=\alpha^{\prime}+\alpha_{n-1},
$$

where $\left.\alpha^{\prime}\right|_{U}=\sum_{\lambda \in O_{4}} \lambda$, in particular, $\alpha^{\prime}$ is irreducible over $P$. Arguing as above, we see that $a_{1}+b_{1}=1, a_{2}=b_{2}=0$, i.e., $\operatorname{Sym}^{2}\left(\alpha_{n}\right)-1_{G}$ is reducible and $\Lambda^{2}\left(\alpha_{n}\right)$ is irreducible. Also, (7.8) and (7.9) imply that $4=$ $\left(\left.\left(\alpha_{n}+\beta_{n}\right)\right|_{P},\left.\left(\alpha_{n}+\beta_{n}\right)\right|_{P}\right)_{P}=\left(\left(\alpha_{n}+\beta_{n}\right)^{2}, \mu\right)_{G}$. But $\left(\alpha_{n}^{2}, \mu\right)_{G}=\left(\beta_{n}^{2}, \mu\right)_{G}=2$, hence $\left(\alpha_{n} \beta_{n}, \mu\right)_{G}=0$. Thus $a_{7}=b_{7}=0$, i.e., $\alpha_{n} \beta_{n}$ is irreducible.

Now let $g$ be the central involution in $P$. Since $\gamma_{n}(g)=-\left(2^{2 n-1}+1\right) / 3$, the $g$-fixed point subspace $V_{+}$in the representation space $V$ for $\gamma_{n}$ has dimension equal to $\gamma_{n-1}(1)$ and less than $\left|O_{j}\right|$ for any $j>1$. Again, every constituent of $\left.\gamma_{n}\right|_{L}$ is either $\alpha_{n-1}, \beta_{n-1}$, or $\gamma_{n-1}$. Comparing the character degrees we see that $U$ acts trivially on $V_{+}$and $V_{+}$affords the $L$-character $\gamma_{n-1}$. Also, the -1-eigenspace for $g$ on $V$ has to afford the $P$-characters $\gamma^{\prime}$ and $\gamma^{\prime \prime}$, where $\left.\gamma^{\prime}\right|_{U}=\sum_{\lambda \in O_{3}} \lambda$, and $\left.\gamma^{\prime \prime}\right|_{U}=\sum_{\lambda \in O_{4}} \lambda$, in particular, $\gamma^{\prime}$ and $\gamma^{\prime \prime}$ are distinct irreducible $P$-characters. Thus

$$
\left.\gamma_{n}\right|_{P}=\gamma^{\prime}+\gamma^{\prime \prime}+\gamma_{n-1} .
$$

Arguing inductively on $n$, we see that $\gamma_{n}$ has Schur-Frobenius indicator 1, i.e., $c_{1}=1$ and $c_{2}=0$. From (7.10) it follows that $3=\left(\left.\gamma_{n}\right|_{P},\left.\gamma_{n}\right|_{P}\right)_{P}=$ $\left(\gamma_{n}^{2}, \mu\right)_{G}$, and so $a_{5}+b_{5}+a_{6}+b_{6}=2$. From (7.9) and (7.10) one obtains $1 \geq\left(\left.\alpha_{n}\right|_{P},\left.\gamma_{n}\right|_{P}\right)_{P}=\left(\alpha_{n} \gamma_{n}, \mu\right)_{G}$, i.e., $a_{8}+b_{8} \leq 1$. Similarly, (7.8) and (7.10) imply that $a_{9}+b_{9} \leq 1$. But we know that $a+b=18$, so in fact we have equality in all three previous inequalities. Thus $a_{8}+b_{8}=a_{9}+b_{9}=1$ (which means $\alpha_{n} \gamma_{n}$ and $\beta_{n} \gamma_{n}$ are reducible), $\alpha^{\prime}=\gamma^{\prime \prime}$ and $\beta^{\prime}=\gamma^{\prime}$. Since $a_{2}=b_{2}=0$, we see that $\left.\Lambda^{2}\left(\gamma^{\prime \prime}\right)\right|_{P}$ does not involve $1_{P}$. Similarly, $a_{4}=b_{4}=0$ implies that $\left.\Lambda^{2}\left(\gamma^{\prime}\right)\right|_{P}$ does not involve $1_{P}$. Recall that $\gamma_{n-1}$ is of type + and $\gamma_{n-1}, \gamma^{\prime}$, and $\gamma^{\prime \prime}$ are distinct irreducibles. Together with (7.10), this implies that $0=\left(\left.\Lambda^{2}\left(\gamma_{n}\right)\right|_{P}, 1_{P}\right)_{P}=\left(\Lambda^{2}\left(\gamma_{n}\right), \mu\right)_{G}$, i.e., $a_{6}+b_{6}=0$ and $\Lambda^{2}\left(\gamma_{n}\right)$ is irreducible. Finally, we see that $a_{5}+b_{5}=2$ and $\operatorname{Sym}^{2}\left(\gamma_{n}\right)$ is reducible. 
Proposition 7.11. Let $G=\mathrm{S}_{2 n}(q)$, q even, $n \geq 3,(n, q) \neq(3,2)$, and $V$ a self-dual nontrivial irreducible $\mathbb{C} G$-module. Then $X(V)$ is reducible for $X \in\left\{\Lambda^{2}\right.$, Sym $\left.{ }^{2}\right\}$ unless $q=2, X=\Lambda^{2}$, and $V$ affords one of the characters $\alpha_{n}, \beta_{n}, \gamma_{n}$.

Proof. The case $G=\mathrm{S}_{8}(2)$ can be checked from [1] so we may also assume $(n, q) \neq(4,2)$. Let $G=\mathrm{S}_{2 n}(q)$ and $V$ a self-dual nontrivial irreducible $\mathbb{C} G$ module such that $X(V)$ is irreducible. By Proposition 5.5, $X=\Lambda^{2}, q \leq 8$, and $\operatorname{dim}(V)<\left(q^{2 n}-1\right) /(q-1)$. Denote $d=\operatorname{dim}(V), e=\operatorname{dim}(X(V))=$ $d(d-1) / 2$, and let $\rho$ and $\chi$ be the character of $V$ and $X(V)$, respectively.

1) Recall that we are assuming $n \geq 3$ if $q \geq 4$ and $n \geq 5$ if $q=2$. Following the proof of Proposition 5.1 and Theorem 5.5 of [25], one can show that $G$ has exactly $4+q / 2$ nontrivial irreducible characters of degree $<\left(q^{2 n}-1\right) /(q-1)$, namely 4 unipotent characters $\alpha_{n}, \beta_{n}, \rho_{1}, \rho_{2}$, labeled by

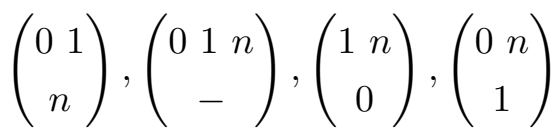

respectively, and $q / 2$ semisimple characters $\gamma_{n}^{i}, 1 \leq i \leq q / 2$.

2) Let $q=8$. By Proposition 5.5, $\operatorname{dim}(V) \leq\left(q^{n}-q\right)^{2} / 2(q-1)$. According to 1$), \rho$ is either $\alpha_{n}$ or $\beta_{n}$. In particular, $d \in 8 \mathbb{Z}+4$, whence $e \in 4 \mathbb{Z}+2$. Suppose that $\chi$ corresponds to a semisimple class $(s)$ in the dual group $G^{*} \simeq G$ and a unipotent character $\psi$ of $C:=C_{G^{*}}(s)$ in Lusztig's Jordan decomposition of characters. One can show that $C$ is a direct product of subgroups of the form $\mathrm{GL}_{m}\left(q^{k}\right), \mathrm{GU}_{m}\left(q^{k}\right)$, and $\mathrm{S}_{2 m}(q)$. By Lemma 7.2, if $\psi(1)>1$ then $\psi(1)$ and so $e$ is divisible by 4 , a contradiction. Hence $\psi(1)=1$ and so $e=\left(G^{*}: C\right)_{2^{\prime}}$ is odd, again a contradiction.

Henceforth we may assume that $q \leq 4$. By Zsigmondy's Theorem [28], if $m \geq 3$ and $(q, m) \neq(2,6)$, then $q^{m}-1$ has a prime divisor which is coprime to $\prod_{i=1}^{m-1}\left(q^{i}-1\right)$. We will denote such a prime by $\ell_{m, q}$.

3) Assume that $q=4$ and $\rho$ is either $\rho_{1}$ or $\rho_{2}$. Then $d-1$ is divisible by $\ell_{4 n-2,2}$ or $\ell_{2 n-1,2}$ respectively. Neither of these primes divides $|G|$, a contradiction. Thus $d=\left(4^{2 n}-1\right) / 5,\left(4^{n}+1\right)\left(4^{n}+4\right) / 10$ or $\left(4^{n}-1\right)\left(4^{n}-4\right) / 10$. In particular, $e$ is odd. Using Lemma 7.2 and arguing as in 2), we see that $\chi$ is a semisimple character and $e=\left(G: C_{G}(s)\right)_{2^{\prime}}$ for some semisimple element $1 \neq s \in G$. First suppose that $3 \leq n \leq 5$. The condition $e$ divides $|G|$ implies that $(n, d, e)=(3,442,97461)$ or $(4,6426,20643525)$. In neither case has $G$ a semisimple element $s$ such that $e=\left(G: C_{G}(s)\right)_{2^{\prime}}$. Hence we may assume that $n \geq 6$. Then $e$ is coprime to $\ell_{2 n-4,4}$.

In the case $q=2$, the characters $\alpha_{n}, \beta_{n}$, and $\gamma_{n}$ have already been treated in Proposition 7.4, hence we may assume that $\rho$ is either $\rho_{1}$ or $\rho_{2}$. Since $e$ does not divide $|G|$ when $n=5$, we must have $n \geq 6$, in which case $e$ is odd and coprime to $\ell_{2 n-4,2}$. Now Lemma 7.2 implies that $\chi$ is not unipotent, and so $\chi$ corresponds to a semisimple element $s \neq 1$ in $G \simeq G^{*}$. 
Thus in either case we obtain a semisimple element $1 \neq s \in G$ such that the $2^{\prime}$-part of the index of $C:=C_{G}(s)$ in $G$ is coprime to $\ell_{2 n-4, q}$ and smaller than $q^{4 n-2}$. Recall that $C$ is a direct product of subgroups of the form $\mathrm{GL}_{m}\left(q^{k}\right)$ or $\mathrm{GU}_{m}\left(q^{k}\right)$ with $m k \leq n$, or $\mathrm{S}_{2 m}(q)$ with $m \leq n-1$. Since $|C|$ is divisible by $\ell_{2 n-4, q}$, it follows that $C$ has a subgroup $D=\operatorname{GU}_{m}\left(q^{k}\right)$ with $m k \geq n-2$ or $\mathrm{S}_{2 m}(q)$ with $m=n-2, n-1$. Also, we are assuming that $n \geq 6$.

Assume $D=\mathrm{GU}_{m}\left(q^{k}\right)$. If $m k=n$ and $n \geq 7$ or $(n, k) \neq(6,1)$ then $(G: C)_{2^{\prime}} \geq q^{4 n-2}$, a contradiction. If $m=n=6$ then $(G: C)$ is divisible by $\ell_{2 n-4, q}$, again a contradiction. If $m k=n-j$ with $j=1,2$, then $C \leq$ $\mathrm{S}_{2 j}(q) \times \mathrm{GU}_{m}\left(q^{k}\right)$, whence $(G: C)_{2^{\prime}} \geq q^{4 n-2}$, a contradiction.

Thus $D=\mathrm{S}_{2 n-2 j}(q)$ with $j=1$ or 2 . Therefore $C \leq \mathrm{S}_{2 j}(q) \times \mathrm{S}_{2 n-2 j}(q)$. If $q=4$, then, as shown above, $e=(G: C)_{2^{\prime}}$, whence $\left(4^{2 n}-1\right)\left(4^{2 n-2}-1\right)$ is divisible by $e=\left(4^{2 n}-1\right)\left(4^{2 n}-6\right) / 50,\left(4^{2 n}-1\right)\left(4^{n}+4\right)\left(4^{n}+6\right) / 200$, or $\left(4^{2 n}-1\right)\left(4^{n}-4\right)\left(4^{n}-6\right) / 200$, a contradiction. If $q=2$, then $45 \cdot(G: C)_{2^{\prime}}$ is divisible by $2^{2 n}-1$, which implies that $2^{2 n}-1$ divides $45 e$, with $e=$ $\left(2^{n}+1\right)\left(2^{n-1}-1\right)\left(2^{n-2}\left(2^{n}-1\right)-1\right)$ or $e=\left(2^{n}-1\right)\left(2^{n-1}+1\right)\left(2^{n-2}\left(2^{n}+1\right)-1\right)$, again a contradiction.

Proposition 7.12. Let $G=\mathrm{O}_{2 n}^{\epsilon}(q), n \geq 4, q$ even, and $V$ a self-dual nontrivial irreducible $\mathbb{C} G$-module. Then $X(V)$ is reducible for $X \in\left\{\Lambda^{2}, \mathrm{Sym}^{2}\right\}$.

Proof. 1) The case $\mathrm{O}_{8}^{ \pm}(2)$ can be checked directly, hence we may assume that $(n, q) \neq(4,2)$. Assume that $X(V)$ is irreducible. Denote $d=\operatorname{dim}(V)$, $e=\operatorname{dim}(X(V))=d(d \pm 1) / 2$, and let $\rho$ and $\chi$ be the character of $V$ and $X(V)$, respectively. By Proposition 5.8, $q=2,4$ and $d<q^{2 n-2}$. Applying Propositions 7.1 and 7.2 of [25] in the case $\rho$ is unipotent, and following the proof of Theorem 7.6 of [25] in the non-unipotent case, we conclude that either $\rho$ is the smallest unipotent character $\rho_{n}$ of degree $\left(q^{n}-\epsilon\right)\left(q^{n-1}+\right.$ $\epsilon q) /\left(q^{2}-1\right)$, or it is one of $q / 2$ semisimple characters $\vartheta_{n}^{i}, 1 \leq i \leq q / 2$, of degree $\left(q^{n}-\epsilon\right)\left(q^{n-1}-\epsilon\right) /(q+1)$, or $G=\mathrm{O}_{8}^{+}(4)$ and $\rho(1)=3213$.

2) Suppose $q=4$. First we consider the case $G=\mathrm{O}_{8}^{+}(4)$. Then either $d=1156$ or $d=3213$, and in either case neither $d-1$ nor $d+1$ divides $|G|$, a contradiction. Hence we may assume that $(n, \epsilon) \neq(4,+)$. Under this assumption, the proof of Proposition 5.8 shows that the restriction of $\rho$ to the parabolic subgroup $P=U . L$ (in the notation of that proof) contains a linear character of the elementary abelian 2-group $U$ with multiplicity 1 . Since this linear character obviously is of type,$+ \rho$ itself is of type + , and so $X=\Lambda^{2}, e=d(d-1) / 2$. Since $\rho$ is either $\rho_{n}$ or $\vartheta_{n}^{i}$, we see that $e \in 4 \mathbb{Z}+2$. Assume that under the Jordan decomposition $\chi$ corresponds to a semisimple class $(s)$ in $G^{*} \simeq G$ and a unipotent character $\psi$ of $C:=C_{G^{*}}(s)$. One can show that $C$ is a direct product of subgroups of the form $\mathrm{GL}_{m}\left(q^{k}\right), \mathrm{GU}_{m}\left(q^{k}\right)$ or $\mathrm{O}_{2 m}^{ \pm}(q)$. Hence by Lemma $7.2 \psi(1)$ is either 1 or divisible by 4 . Thus $e=\left(G^{*}: C\right)_{2^{\prime}} \psi(1)$ cannot belong to $4 \mathbb{Z}+2$, again a contradiction. 
3) We are reduced to consider $q=2$. The case $G=\mathrm{O}_{10}^{ \pm}(2)$ can be checked directly. Therefore we are left with the case $q=2, n \geq 6$, and $\rho$ is either $\rho_{n}$ or $\vartheta_{n}:=\vartheta_{n}^{1}$. Recall that, in the notation of the proof of Proposition 5.8, the Levi subgroup $L$ of $P$ acts on the nontrivial linear characters of $U$ with two orbits say $O_{i}$ and $O_{a}$ of lengths $\left(2^{n-1}-\epsilon\right)\left(2^{n-2}+\epsilon\right)$ and $2^{n-2}\left(2^{n-1}-\epsilon\right)$, and $O_{i}$ does not occur on $V$. Observe that $d<2\left|O_{a}\right|$ and also $U$ cannot act trivially on $V$. Thus each character from $O_{a}$ occurs on $V$ with multiplicity 1. It follows that $\left.\rho\right|_{P}=\lambda+\beta$, where $\lambda$ is an irreducible $P$-character whose restriction to $U$ is $\sum_{\alpha \in O_{a}} \alpha$, and $\beta$ is an $L$-character inflated to $P$. Also, the type of $\rho$ is + , whence $X=\Lambda^{2}$ and $e=d(d-1) / 2$.

First we suppose that $\rho=\rho_{n}$. Then $\beta$ is an $L$-character of degree $\left(2^{2 n-3}+\right.$ $\left.9 \epsilon 2^{n-2}-2\right) / 3<2^{2 n-4}$ as $n \geq 6$. According to 1$)$ applied to $L=\mathrm{O}_{2 n-2}^{\epsilon}(2)$, $\beta=a \cdot 1_{L}+b \cdot \rho_{n-1}+c \cdot \vartheta_{n-1}$ for some non-negative integers $a, b, c$. Thus

$$
\frac{2^{2 n-3}+9 \epsilon 2^{n-2}-2}{3}=a+b \frac{2^{2 n-3}+3 \epsilon 2^{n-2}-2}{3}+c \frac{2^{2 n-3}-3 \epsilon 2^{n-2}+1}{3} .
$$

Since $a, b, c$ are non-negative integers, we come to the conclusion that $a \geq$ $2^{n-1}$. Hence $2^{n-1} \leq\left(\left.\rho\right|_{P}, 1_{P}\right)_{P}=\left(\rho, 1_{P}^{G}\right)_{G}$, and so $\rho(1) \leq(G: P) / 2^{n-1}$, a contradiction.

Finally, let $\rho=\vartheta_{n}$. Since $e$, and so $|G|$, is divisible by $\left(2^{2 n}-1\right) / 3$, we must have that $\epsilon=-$ and $n \geq 6$ is even. Since $e$ is odd, $\chi$ cannot be unipotent by Lemma 7.2. Suppose that $\chi$ corrersponds to a semisimple class $(s)$ in $G$ and a unipotent character $\psi$ of $C:=C_{G}(s)$. One can show that either $C \leq \mathrm{GU}_{2}(2) \times \mathrm{O}_{2 n-4}^{-}(2)$, or $C$ is a direct product of subgroups of the forms $\mathrm{GL}_{m}\left(q^{k}\right), \mathrm{GU}_{m}\left(q^{k}\right), \mathrm{O}_{2 m}^{ \pm}(q)$. In the former case $(G: C)_{2^{\prime}}$ is divisible by $\ell_{n-1,2}$ if $n \neq 7$, and by 31 if $n=7$, meanwhile $e$ is not, a contradiction. In the latter case, the oddness of $e$ and Lemma 7.2 imply that $e=(G: C)_{2^{\prime}}$. In the case $n=6$, one can show directly that $G=\mathrm{O}_{12}^{-}(2)$ has no such $C$ with $e=(G: C)_{2^{\prime}}=255255$. Therefore we assume that $n \geq 8$ is even. In this case, $e$ is not divisible by $\ell_{2 n-6,2}$, whence $C$ must contain a subgroup $D$ of the form $\mathrm{GU}_{m}\left(q^{k}\right)$ with $m k \geq n-3$ or $\mathrm{O}_{2 m}^{ \pm}(q)$ with $n-3 \leq m \leq n-1$. Assume $D=\mathrm{GU}_{m}\left(q^{k}\right)$. If $m k=n$, then $|C|$ is divisible by $2^{n} \pm 1$, and so $(G: C)_{2^{\prime}}$ is not divisible by $\ell_{2 n, 2}$ or $\ell_{n, 2}$, while $e$ is divisible by that prime, a contradiction. If $m k=n-1$, then $e$, but not $(G: C)_{2^{\prime}}$, is divisible by $\ell_{2 n-2,2}$. If $m k=n-2$ or $n-3$, then $(G: C)_{2^{\prime}}$, but not $e$, is divisible by $\ell_{n-1,2}$. Assume $D=\mathrm{O}_{2 m}^{ \pm}(q)$. If $m=n-3$ or $n-2$, then $(G: C)_{2^{\prime}}$, but not $e$, is divisible by $\ell_{n-1,2}$. If $m=n-1$, then $e$, but not $(G: C)_{2^{\prime}}$, is divisible by $\ell_{2 n-4,2}$.

\section{B. The exceptional groups in characteristic $\ell=0$.}

For the exceptional groups we can obtain a complete answer in characteristic 0 thanks to the tables of low-dimensional irreducible representations compiled by Frank Lübeck [17]. These in turn again rely on Lusztig's classification of irreducible characters of finite reductive groups. 
Proposition 7.13. Let $G$ be a quasi-simple exceptional group of Lie type and $V$ a nontrivial irreducible $\mathbb{C} G$-module. Then $X(V)$ is reducible unless possibly if $(G, \operatorname{dim}(V), X)$ are as in Table 7.15.

Proof. By Theorem 3.1 and the remark after its proof we may assume that $q$ is even and we are in the bad case. The cases ${ }^{2} B_{2}\left(q^{2}\right)$ and $G_{2}(q)$ were treated in 5A. Now assume that $G={ }^{3} D_{4}(q)$. By [17] the smallest degree of a nontrivial complex irreducible representation of $G$ is $q\left(q^{4}-q^{2}+1\right)$, while the next largest is $d_{2}=q^{3}(q-1)^{2}\left(q^{4}-q^{2}+1\right) / 2$ (since $q$ is even). Using [1] we may moreover assume that $q>2$. Then $d_{2}\left(d_{2}-1\right) / 2$ is already larger than the largest degree of an irreducible complex character of $G$, hence only the smallest nontrivial character of $G$ remains. But $\operatorname{dim}(X(V))$ involves a factor $q^{4} \pm q^{3}+1$ which does not divide the order of $G$.

For $G={ }^{2} F_{4}\left(q^{2}\right)^{\prime}$ or $G=F_{4}(q)$ the cases $q^{2}=2$ respectively $q=2$ can be dealt with by [1]. Otherwise Proposition 5.15 gives $\operatorname{dim}(V) \leq 2 q^{11}(q+1)$. By the tables in [17] this forces $\operatorname{dim}(V)=\sqrt{2} q\left(q^{4}-1\right)\left(q^{6}+1\right) / 2$ respectively

$$
\operatorname{dim}(V) \in\left\{\frac{1}{2} q\left(q^{3}-1\right)^{2}\left(q^{4}+1\right), \frac{1}{2} q\left(q^{4}+1\right)\left(q^{6}+1\right), \frac{1}{2} q\left(q^{3}+1\right)^{2}\left(q^{4}+1\right)\right\} .
$$

In the first case the precise power of 2 dividing $\operatorname{dim}(X(V))$ is $\sqrt{2} q / 4$, but ${ }^{2} F_{4}\left(q^{2}\right)$ does not have an irreducible character with this property unless $q^{2}=8$. For $q^{2}=8$ it is readily checked that $\operatorname{dim}(X(V))$ does not divide $\left|{ }^{2} F_{4}(8)\right|$. In the second case, the precise power of $q$ dividing $\operatorname{dim}(X(V))$ is $q / 4$. If $q>4$ then $F_{4}(q)$ does not have such a character. For $q=4$ the dimension $\operatorname{dim}(X(V))$ does not divide $|G|$.

Now assume that $G={ }^{2} E_{6}(q)$ or $G=E_{n}(q)$ with $n=6,7,8$. We may treat the covering groups of ${ }^{2} E_{6}(2)$ as follows: The faithful characters of coverings with center of order divisible by 3 cannot be self-dual. The remaining characters are printed in [1]. By Proposition 5.14 and [17] we are reduced to the case where the character $\chi$ of $V$ is the smallest nontrivial one of $G$, that is, $\chi=\phi_{2,4}^{\prime}, \phi_{6,1}, \phi_{7,1}, \phi_{8,1}$ respectively, or $q=2$. In the first case, $\operatorname{dim}(X(V))$ does not divide $|G|$. For $G=E_{6}(2)$ the tables in [17] show that only $\operatorname{dim}(V) \in\{2482,137020,443548\}$ are below $\sqrt{2 \sqrt{|G|}}$, and for any of them $\operatorname{dim}(X(V))$ does not divide $|G|$. Similarly, for $G=E_{7}(2)$ only the first five characters, of degrees 141986, 86507701, 95420052, 181785768, 2422215628 can occur, but for none of these $\operatorname{dim}(X(V))$ divides $|G|$. For $G=E_{8}(2)$ only the first seven characters, of degrees 545925250, 76321227908420, 46453389380074796, 51320060161363500, $97697128859455125,144074197011621500,148940867792910204$ can occur, but for none of these $\operatorname{dim}(X(V))$ is the degree of an irreducible character of $G$. (The authors are thankful to Lübeck for kindly providing them with the table of character degrees for $E_{8}(2)$.) 
Thus, together with the results from [18] we have completed the case $\ell=0$ (see also $[20]$ for the case $X=\widetilde{A}$ ):

Theorem 7.14. Let $G$ be a quasi-simple group and let $S:=G / Z(G)$. Suppose that $G$ has a nontrivial irreducible $\mathbb{C} G$-module $V$ such that $X(V)$ is irreducible, where $X=\widetilde{A}$ if $V$ is not self-dual and $X=\widetilde{\Sigma}^{2}$ or $\widetilde{\Lambda}^{2}$ otherwise. Then one of the following holds.

(i) $S=\mathfrak{A}_{n}$ and $V$ is the heart of the natural permutation module.

(ii) $S=\mathrm{S}_{2 n}(q), q=3,5,9, V$ is a Weil module of $\operatorname{Sp}_{2 n}(q)$ of degree $\left(q^{n} \pm 1\right) / 2$.

(iii) $S=\mathrm{S}_{2 n}(2), X=\Lambda^{2}$, and the character of $V$ is one of the unipotent

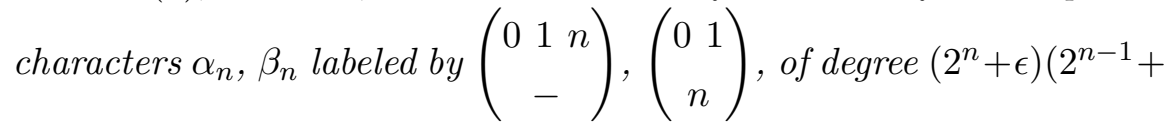
$\epsilon) / 3$ with $\epsilon= \pm 1$, or a (unique) semisimple character $\gamma_{n}$ of degree $\left(2^{2 n}-1\right) / 3$.

(iv) $S=\mathrm{U}_{n}(q), q=2,3$, and $V$ is a Weil module of $\mathrm{SU}_{n}(q)$ of degree $\left(q^{n}+q(-1)^{n}\right) /(q+1),\left(q^{n}-(-1)^{n}\right) /(q+1)$.

(v) "Small groups": $(G, \operatorname{dim}(V), X)$ is as in Table 7.15.

\section{C. A question of Gross.}

B.H. Gross asked the question which finite subgroups $G$ of complex simple simply-connected Lie groups $\mathfrak{G}$ have the property that they act irreducibly in all fundamental representations of $\mathfrak{G}$. A well-known family of examples is provided by the finite irreducible complex reflection groups: All exterior powers of their reflection representations remain irreducible, thus they give examples where $\mathfrak{G}=\mathrm{SL}_{n}(\mathbb{C})$ (see for example $\mathfrak{S}_{n+1}<\mathrm{SL}_{n}(\mathbb{C})$ ).

Clearly, if $G$ has the above mentioned property, then so has the product of $G$ with any subgroup $Z$ of the centre of $\mathfrak{G}$. We adopt the following notation: If $G<\mathfrak{G}=\operatorname{Spin}_{d}(\mathbb{C})$ then we write $\bar{G}$ for the image of $G$ in $\mathrm{SO}_{d}(\mathbb{C})$, and otherwise set $\bar{G}=G$.

We start our investigation by reducing the general case of Gross' question to the monomial and the almost quasi-simple case, which will then be treated subsequently.

Theorem 7.16. Let $G$ be a finite subgroup of the simple simply-connected complex Lie group $\mathfrak{G}$ which is irreducible in all fundamental representations of $\mathfrak{G}$. Assume that the dimension d of the natural module $V$ for $\mathfrak{G}$ is at least 5. Then up to a finite subgroup of $Z(\mathfrak{G})$ one of the following holds.

(i) $\bar{G}$ is an irreducible monomial group in $\mathrm{GL}(V)$.

(ii) $G=2^{3} \cdot \mathrm{SL}_{3}(2)$ and $\mathfrak{G}=G_{2}(\mathbb{C})$.

(iii) $G \leq 5_{+}^{1+2}: \mathrm{SL}_{2}(5)$ and $\mathfrak{G}=\mathrm{SL}_{5}(\mathbb{C})$. 
Table 7.15. Non-generic examples for $\ell=0$.

\begin{tabular}{|l|r|rrr|}
\hline$G$ & $\operatorname{dim}(V)$ & $\widetilde{\Lambda}^{2}$ & $\widetilde{\Sigma}^{2}$ & $\widetilde{A}$ \\
\hline $3 . \mathfrak{A}_{6}$ & 3 & & & $\times$ \\
$2 . \mathfrak{A}_{7}$ & 4 & & & $\times$ \\
$6 . \mathfrak{A}_{7}$ & 6 & & & $\times$ \\
$2 . \mathfrak{A}_{8}$ & 8 & & $\times$ & \\
$2 . \mathfrak{A}_{9}$ & 8 & $\times$ & $\times$ & \\
\hline $\mathrm{L}_{2}(7)$ & 3 & & & $\times$ \\
$6 . \mathrm{L}_{3}(4)$ & 6 & & & $\times$ \\
$4_{1} \cdot \mathrm{L}_{3}(4)$ & 8 & & & $\times$ \\
\hline $\mathrm{U}_{3}(4)$ & 12 & $\times$ & & \\
$6{ }_{1} \cdot \mathrm{U}_{4}(3)$ & 6 & & & $\times$ \\
$2 . \mathrm{U}_{6}(2)$ & 56 & $\times$ & & \\
\hline $\mathrm{S}_{4}(4)$ & 18 & $\times$ & & \\
$\mathrm{S}_{6}(2)$ & 7 & $\times$ & $\times$ & \\
$2 . \mathrm{S}_{6}(2)$ & 8 & & $\times$ & \\
\hline $2 . \mathrm{O}_{8}^{+}(2)$ & 8 & $\times$ & $\times$ & \\
\hline$G_{2}(3)$ & 14 & $\times$ & $\times$ & \\
$2 . G_{2}(4)$ & 12 & $\times$ & $\times$ & \\
$2 F_{4}(2)^{\prime}$ & 26 & & & $\times$ \\
$2 . F_{4}(2)$ & 52 & $\times$ & $\times$ & \\
$2 .{ }^{2} E_{6}(2)$ & 2432 & $\times$ & & \\
\hline $\mathrm{M}_{11}$ & 10 & $\times$ & & \\
$\mathrm{M}_{11}$ & 11 & $\times$ & & \\
$2 . \mathrm{M}_{12}$ & 10 & & & $\times$ \\
$\mathrm{M}_{12}$ & 11 & $\times$ & & \\
\hline
\end{tabular}

(iv) $\bar{G} \leq 2_{+}^{1+6} \cdot \mathfrak{S}_{8}$ and $\mathfrak{G}=\operatorname{Spin}_{8}(\mathbb{C})$.

(v) $G$ is almost quasi-simple.

Proof. 1) First we assume that $\mathfrak{G}$ is an exceptional group. Suppose $G$ is not Lie primitive (in the sense of [4]). Then there is a proper closed subgroup $\mathfrak{H}$ of $\mathfrak{G}$ which contains $G$, whence $\mathfrak{H}$ and $G$ are not irreducible on the adjoint fundamental representation. So $G$ is Lie primitive. By Theorem 1.7 of [4], either we are in Case (v) or (the image of) $G$ in $\mathfrak{G}_{a d}$ is contained in the 
Table 7.15. Non-generic examples for $\ell=0$ (continued).

\begin{tabular}{|l|r|lll|}
\hline$G$ & $\operatorname{dim}(V)$ & $\widetilde{\Lambda}^{2}$ & $\widetilde{\Sigma}^{2}$ & $\widetilde{A}$ \\
\hline $2 . \mathrm{M}_{12}$ & 12 & $\times$ & & \\
$2 . \mathrm{M}_{22}$ & 10 & & & $\times$ \\
$\mathrm{M}_{22}$ & 21 & $\times$ & & \\
$2 . \mathrm{J}_{2}$ & 6 & $\times$ & $\times$ & \\
$2 . \mathrm{J}_{2}$ & 14 & $\times$ & & \\
$\mathrm{M}_{23}$ & 22 & $\times$ & & \\
$\mathrm{M}_{23}$ & 45 & & & $\times$ \\
$\mathrm{HS}$ & 22 & $\times$ & & \\
$3 . \mathrm{J}_{3}$ & 18 & & & $\times$ \\
$\mathrm{M}_{24}$ & 23 & $\times$ & & \\
$\mathrm{M}_{24}$ & 45 & & & $\times$ \\
$\mathrm{McL}_{2}$ & 22 & $\times$ & $\times$ & \\
$2 . \mathrm{Ru}$ & 28 & & & $\times$ \\
$6 . \mathrm{Suz}$ & 12 & & & $\times$ \\
$\mathrm{Co}_{3}$ & 23 & $\times$ & $\times$ & \\
$\mathrm{Co}_{3}$ & 253 & $\times$ & & \\
$\mathrm{Co}_{2}$ & 23 & $\times$ & $\times$ & \\
$3 . \mathrm{ON}$ & 342 & & & $\times$ \\
$\mathrm{Fi}_{22}$ & 78 & $\times$ & $\times$ & \\
$\mathrm{HN}$ & 133 & $\times$ & $\times$ & \\
$\mathrm{Th}_{2}$ & 248 & $\times$ & $\times$ & \\
$2 . \mathrm{Co}{ }_{1}$ & 24 & $\times$ & $\times$ & \\
$J_{4}$ & 1333 & & & $\times$ \\
$\mathrm{B}$ & 4371 & $\times$ & & \\
\hline
\end{tabular}

normalizer $N=N_{\mathfrak{G}}(J)$ of a so-called Jordan subgroup $J$. If $\mathfrak{G}=G_{2}(\mathbb{C})$, then $J=2^{3}$ and $N=2^{3} \cdot \mathrm{SL}_{3}(2)$. One can check that $N$ is irreducible on both fundamental representations of $\mathfrak{G}$, and no proper subgroup of $N$ has this property, so $G=N$. In the other cases, $|N|$ is not divisible by $7,5,19$, respectively, whence $N$ and $G$ cannot act irreducibly on the fundamental representation of degree 273 , resp. 2925,147250 , of $\mathfrak{G}=F_{4}(\mathbb{C})$, resp. $E_{6}(\mathbb{C})$, $E_{8}(\mathbb{C})$. 
From now on we assume that $\mathfrak{G}$ is classical. We apply the main result of [15] to $\bar{G}$ and see that we are in case (v) or $\bar{G}$ is in one of the families $\mathcal{C}_{j}$ with $1 \leq j \leq 6$ defined in [15].

Suppose that $\bar{G}$ is in $\mathcal{C}_{6}$. Then $\mathfrak{G}=\operatorname{SL}(V), \bar{G}=G \leq N_{\mathfrak{G}}(\mathfrak{H})=Z * \mathfrak{H}$, where $Z=Z(\mathfrak{G})$ and $\mathfrak{H}=\operatorname{Sp}(V)$ or $\operatorname{SO}(V)$. Set $H=Z G \cap \mathfrak{H}$. Clearly $Z G=Z * H$, and the finite subgroups $Z G$ and $H$ also act irreducibly on all fundamental representations of $\mathfrak{G}$. At this point we may apply [15] again to the subgroup $H$ of $\mathfrak{H}$. Thus we may assume that $\bar{G} \in \mathcal{C}_{j}$ with $j \leq 5$. Moreover, $j>1$ since $G$ is irreducible on the natural module $V$ of $\mathfrak{G}$.

2) Assume $\bar{G}$ is in $\mathcal{C}_{2} \cup \mathcal{C}_{3}$, i.e., $\bar{G}$ preserves a direct sum decomposition $V=\oplus_{i=1}^{m} V_{i}$ with $\operatorname{dim}\left(V_{i}\right)=e$ and $m>1$. Then $W^{\prime}:=\oplus_{i} \Lambda^{2}\left(V_{i}\right)$ is a $\bar{G}$ submodule of $W:=\Lambda^{2}(V)$. If $e \geq 2$, then $2 \leq \operatorname{dim}\left(W^{\prime}\right) \leq \operatorname{dim}(W)-2$. On the other hand, the second fundamental representation of $\mathfrak{G}$ is of codimension $\leq 1$ in $W$. Therefore $\bar{G}$ cannot act irreducibly on this representation, a contradiction. Hence $e=1$, which means $\bar{G}$ is an irreducible monomial group in $\mathrm{GL}(V)$.

3) Next assume that $\bar{G}$ is in $\mathcal{C}_{5}$, that is, $\bar{G} \leq N:=N_{\mathfrak{G}}(E)$ where either $E$ is an extraspecial $r$-group of order $r^{2 m+1}$, or $Z_{2^{t}} * 2_{+}^{1+2 m}$. In particular, $d=r^{m}$, resp. $2^{m}$.

Suppose $d=5$, so $r=5$. Then $\mathfrak{G}=\mathrm{SL}_{5}(\mathbb{C})$. Clearly $N$ acts irreducibly on $V$ and $\Lambda^{4}(V)$. We claim that $N$ is also irreducible on $\Lambda^{2}(V)$ and so on $\Lambda^{3}(V)$ as well. Assume $N$ is reducible on $\Lambda^{2}(V)$. Then the character $\chi$ of $N$ on this module is a sum of two faithful irreducible characters of degree 5. Each of them restricted to $\mathrm{SL}_{2}(5)$ is a reducible Weil character of degree 5 and so it takes value 1 on the central involution $z$ of $\mathrm{SL}_{2}(5)$. Thus $\chi(z)=2$. On the other hand, $z$ acts on $\Lambda^{2}(V)$ with trace -2 , a contradiction. Consequently, $N$ is irreducible on all fundamental representations of $\mathfrak{G}$. Now $G$ can be any subgroup of $N$ of the form $E: H$, where $H$ is a subgroup of $\mathrm{SL}_{2}(5)$ which acts irreducibly on a 2-dimensional complex representation of $\mathrm{SL}_{2}(5)$.

Suppose $d=7$. Then $\mathfrak{G}=\mathrm{SL}_{7}(\mathbb{C}), G \leq 7_{+}^{1+2}: \mathrm{SL}_{2}(7)$, and 5 does not divide $|G|$, so $G$ is not irreducible on $\Lambda^{3}(V)$.

Suppose $d=8$. Then $\mathfrak{G}=\operatorname{Sp}_{8}(\mathbb{C}), \mathrm{SL}_{8}(\mathbb{C})$ or $\operatorname{Spin}_{8}(\mathbb{C})$. In the first case, the order of $N=2_{-}^{1+6} \cdot \mathrm{O}_{6}^{-}(2)$ is not divisible by 7 , so $N$ is reducible on the fourth fundamental representation of $\mathrm{Sp}_{8}(\mathbb{C})$. In the second case, the character of $E$ on $\Lambda^{4}(V)$ is a sum of some linear characters of $E$, at least two of which are distinct. Since $N$ is irreducible on $\Lambda^{4}(V)$, this sum is $70 / s$ times the sum of $s \geq 2$ distinct linear characters of $E$. On the other hand, $N / E \simeq \operatorname{Sp}_{6}(2)$ does not have a subgroup of such an index $s$, a contradiction. So we are in the third case: $N=2_{+}^{1+6} \cdot \mathfrak{S}_{8}$. Clearly, $N$ acts irreducibly on $V$. $N$ is also irreducible on $\Lambda^{2}(V)$, since $N$ acts transitively on 28 linear characters of $E$ occurring on $\Lambda^{2}(V)$. The third and the fourth fundamental representations of $\operatorname{Spin}_{8}(\mathbb{C})$ have kernel $Z_{2}$ and dimension 8 . 
So $E$ acts nontrivially on them. If $Z(E)$ is nontrivial on any of them, then $E$ is irreducible on that representation. On the other hand, if $Z(E)$ acts trivially on one of them, then $N$ is irreducible on it since $N$ is transitive on the linear characters of $E$ occurring on it. Thus $N$ is indeed irreducible on all fundamental representations. A similar argument shows that $G$ has this property if $G=E \cdot H$ where $H$ is any 2-transitive subgroup of $\mathfrak{S}_{8}$.

Suppose $d \geq 9$. If $d \neq 11$ then by Lemma 7.19 there is a prime $p$ such that $[d / 2]+3 \leq p \leq d-2$ and $p$ divides the degree of a fundamental representation of $\mathfrak{G}$, see the proof of Proposition 7.21. If $d=11$ then $\mathfrak{G}=\mathrm{SL}_{11}(\mathbb{C})$, and 7 divides the degree of the fifth fundamental representation of $\mathfrak{G}$, so we may choose $p=7$. By assumption $p$ divides $|G|$, and clearly $p \neq r$. So $p$ divides the order of $\bar{G} / E \leq \operatorname{Sp}_{2 m}(r)$. Such a prime is either $\leq r^{m-1}+1 \leq d / 2+1$, or $\leq\left(r^{m}+1\right) / 2=(d+1) / 2$, or equal to $r^{m} \pm 1=d \pm 1$. Any of these (in)equalities contradicts the choice of $p$.

4) Let $\bar{G}$ be in $\mathcal{C}_{4}$ (i), i.e., $\bar{G}$ preserves a tensor product decomposition $V=V_{1} \otimes V_{2}$, with $\operatorname{dim}\left(V_{i}\right)=e_{i}>1$ and $e_{2} \geq e_{1}$.

First assume that $d>6$ and $d \neq 9$. Then $1<e_{1} \leq d / 2-2$. If $\mathfrak{G}$ is not of type $C$, then the fixed point subspace $F$ for $\mathrm{SL}\left(V_{1}\right)$ on the fundamental representation $\Lambda^{e_{1}}(V)$ is clearly a nonzero proper $\mathrm{GL}\left(V_{1}\right) \otimes \mathrm{GL}\left(V_{2}\right)$-submodule, a contradiction. If $\mathfrak{G}$ is of type $C$, then we may assume $\bar{G} \leq \mathrm{O}\left(V_{1}\right) \otimes \operatorname{Sp}\left(V_{2}\right)$. Fix a nonzero singular vector $e \in V_{2}$. Then $V^{\prime}=V_{1} \otimes e$ is totally singular, whence $0 \neq U:=\Lambda^{e_{1}}\left(V^{\prime}\right)$ is contained in the kernel of the contraction map $\Lambda^{e_{1}}(V) \rightarrow \Lambda^{e_{1}-2}(V)$. Thus $U$ is contained in the $e_{1}$ th fundamental representation of $\mathfrak{G}$, and clearly $U$ is fixed by $\mathrm{SO}\left(V_{1}\right)$ pointwise. Now we can repeat the above argument with $\mathrm{SO}\left(V_{1}\right)$ instead of $\mathrm{SL}\left(V_{1}\right)$.

Observe that this argument also works if $d=9$, or if $d=6$ and $\mathfrak{G} \neq$ $\operatorname{Spin}_{6}(\mathbb{C})$, as $\Lambda^{e_{1}}(V)$ is a fundamental representation for $\mathfrak{G}$. The case $\operatorname{Spin}_{6}(\mathbb{C})$ can be viewed as $\mathrm{SL}_{4}(\mathbb{C})$, in which case we apply the same argument to $\Lambda^{2}(V)$.

5) Finally, let $\bar{G}$ be in $\mathcal{C}_{4}$ (ii), i.e., $\bar{G}$ preserves a tensor power decomposition $V=U_{1} \otimes U_{2} \otimes \ldots \otimes U_{m}$, with $U_{i} \simeq U$ and $\operatorname{dim}(U)=e>1$. Fix a Borel subgroup $B_{1}$ in $\mathrm{GL}\left(U_{1}\right)$ and a nonzero singular $B_{1}$-invariant subspace $\langle v\rangle_{\mathbb{C}}$. Set $V^{\prime}=v \otimes U_{2} \otimes \ldots \otimes U_{m}$. Then $V^{\prime}$ is totally singular, so as in 4), $T:=\Lambda^{s}\left(V^{\prime}\right)$ is contained in the $s^{\text {th }}$ fundamental representation $W$ of $\mathfrak{G}$, where $s=e^{m-1}$. Also, $T$ is one-dimensional and invariant under $B:=B_{1} \otimes \operatorname{GL}\left(U_{2}\right) \otimes \ldots \otimes \operatorname{GL}\left(U_{m}\right)$. Thus $T$ is a highest weight subspace for $B$ (affording the highest weight $s \omega_{1}$ for $B_{1}$ ), so $W$ contains a nonzero $H$-submodule say $W_{1}$ of dimension at most $e^{s}$, where $H=\mathrm{GL}\left(U_{1}\right) \otimes \ldots \otimes \mathrm{GL}\left(U_{m}\right)$. Repeating the same argument for $i$ instead of 1 we get a nonzero $H$-submodule $W_{i}, i=1, \ldots, m$. Observe that $\mathfrak{S}_{m}$ permutes the $W_{i}$ 's, so $W^{\prime}:=\sum_{i} W_{i}$ is a $\bar{G}$-submodule of dimension at most $m e^{s}$, as $\bar{G} \leq H: \mathfrak{S}_{m}$. Now if $d=e^{m} \neq 8$ then $m e^{s}<\operatorname{dim}(W)$, a contradiction. If $d=8$ then $m=3, e=2$, and instead of $\operatorname{dim}\left(W_{i}\right) \leq 16$ we have 
the better bound $\operatorname{dim}\left(W_{i}\right) \leq 11$ (as $W_{1}$ is a $\operatorname{GL}\left(U_{1}\right)$-submodule of $U_{1}^{\otimes 4}$ and $\left.\operatorname{dim}\left(\operatorname{Sym}^{4}\left(U_{1}\right)\right)=5\right)$, whence $\operatorname{dim}\left(W^{\prime}\right) \leq 33<42 \leq \operatorname{dim}(W)$ as well.

One can say more about the monomial case in this theorem:

Lemma 7.17. In Case (i) of Theorem 7.16 we have $\bar{G}=\bar{E} \cdot H$, where $\bar{E}$ is a normal abelian homocyclic subgroup and either $\mathfrak{A}_{d} \leq H \leq \mathfrak{S}_{d}$, or $d \leq 11$ and $(\mathfrak{G}, H)$ are as in Table 7.18 , where moreover $|\bar{E}|=2^{s}$ with $d-2 \leq s \leq d$ when $\mathfrak{G}=\operatorname{Spin}_{d}(\mathbb{C})$.

Proof. By the proof of Thm. 7.16 we have that $\mathfrak{G}$ is a classical group on $V$, and $\bar{G}$ permutes a basis of $V$ and is of the form $\bar{E} \cdot H$ with $\bar{E}$ a normal abelian subgroup and $H \leq \mathfrak{S}_{d}$.

The set of weight spaces of $\bar{E}$ is a $\bar{G}$-invariant decomposition of $V$, so as in part 2) of the proof of Thm. 7.16, each one is one dimensional (else we violate irreducibility), and $H$ acts transitively on the $\bar{E}$-weight spaces of $V$. By transitivity of $H$ either all weight spaces are singular or non-singular. In the latter case $\mathfrak{G}$ is Spin and $\bar{E}$ is an elementary abelian 2-group and the weight spaces form an orthonormal basis of $V$.

Now we assume that all $\bar{E}$-weight spaces $V_{\chi_{i}}$ of $V$ are singular and one dimensional. If $f$ is the $\mathfrak{G}$-invariant bilinear form and $v_{i} \in V_{\chi_{i}}$, then for all $g$ in $\bar{E}$

$$
f\left(v_{i}, v_{j}\right)=f\left(g v_{i}, g v_{j}\right)=\chi_{i}(g) \chi_{j}(g) f\left(v_{i}, v_{j}\right) .
$$

So $f\left(v_{i}, v_{j}\right)=0$ unless $\chi_{i}(g)=\chi_{j}(g)^{-1}$. As $f$ is nondegenerate there exists for every weight space $V_{\chi_{i}}$ at least one weight space $V_{\chi_{j}}$ outside of the orthogonal complement of $V_{\chi_{i}}$. By the computation above $V_{\chi_{j}}=V_{-\chi_{i}}$. Thus the set of $\bar{E}$-weight spaces is a $G$ invariant hyperbolic basis of $V$. As $\mathfrak{G}$ is transitive on hyperbolic bases, and the normalizer of a hyperbolic basis is the normalizer of a split torus, we get the embedding of $G$ into $N_{\mathfrak{G}}(T)$. But in this case we observe that when $\mathfrak{G}$ is not a linear group, then the zero weight space of the heart of $\Lambda^{2}(V)$ is a proper nontrivial $N_{\mathfrak{G}}(T)$ submodule, by [11], Ex. 13.13. But we assumed that $\bar{G}$ is conjugate to a subgroup of $N_{\mathfrak{G}}(T)$, so $\mathfrak{G}$ must be linear in the second case.

Since $\Lambda^{2}(V)$ is a fundamental representation for $\mathfrak{G}$, it is irreducible, so $H$ is 2-transitive. Note that if $\Lambda^{k}(V)$ is irreducible then $H$ must be a $k$ homogeneous subgroup of $\mathfrak{S}_{d}$. We first deal with the linear case. Since $\Lambda^{r}(V)$ is a fundamental representation of $\mathrm{SL}_{d}(\mathbb{C})$ for any $r \leq d-2$, the group $H$ must act $r$-homogeneously for those $r$. According to [16], a 6homogeneous group is 6 -transitive if $d \geq 12$, hence contains the alternating group. We conclude that in the linear case either $H \geq \mathfrak{A}_{d}$ or $d \leq 11$. Among the transitive groups of degree $5 \leq d \leq 11$ not containing $\mathfrak{A}_{d}$ only $H=5: 4$ with $d=5, H=\mathrm{PGL}_{2}(5)$ with $d=6$, and $H=\mathrm{L}_{2}(8), H=\mathrm{L}_{2}(8): 3$ with $d=9$ are $r$-homogeneous for all $r \leq d-2$. These give rise to examples. 
Now assume that $\mathfrak{G}=\operatorname{Spin}_{d}(\mathbb{C})$. Here $\Lambda^{r}$ is irreducible for $r \leq\lfloor(d-3) / 2\rfloor$, thus $H$ is $\lfloor(d-3) / 2\rfloor$-homogeneous. Arguing as before we conclude that either $d \leq 14$ or $H \geq \mathfrak{A}_{d}$. Among the transitive groups of degree $d \leq 14$ only the following ones are $r$-homogeneous for all $r \leq\lfloor(d-3) / 2\rfloor$ :

$$
\begin{aligned}
d=7: & 7: 3,7: 6, \mathrm{~L}_{3}(2) \\
d=8: & 2^{3}: 7,2^{3}: 7: 3, \mathrm{~L}_{2}(7), \mathrm{PGL}_{2}(7), 2^{3}: \mathrm{L}_{3}(2) \\
d=9: & \mathrm{L}_{2}(8), \mathrm{L}_{2}(8): 3 \\
d=10: & \operatorname{PGL}_{2}(9), M_{10}, \operatorname{Aut}\left(\mathfrak{A}_{6}\right) \\
d=11: & M_{11} \\
d=12: & M_{12} .
\end{aligned}
$$

Now note that, whenever $|\bar{E}| \geq 2^{d-1}$ if $d$ is odd, respectively $|\bar{E}| \geq 2^{d-2}$ if $d$ is even, then $\bar{E}$ lifts to an extraspecial group $E$ in $\operatorname{Spin}_{d}(\mathbb{C})$. This $E$ has a faithful representation of degree equal to the degree of the spin representation, which moreover lifts to the extension $E H$. Hence, for any of the groups $H$ above, whenever $|\bar{E}|$ is large enough we get an example.

Note that the extensions $G=E \cdot H$ in Lemma 7.17 need not necessarily split, as is shown by the example of Weyl groups in $\mathrm{SL}_{n}(\mathbb{C})$.

Table 7.18. Monomial subgroups $G=E . H$ of complex simple Lie groups $\mathfrak{G}$ irreducible in all fundamental representations.

\begin{tabular}{|l|l|}
\hline $\mathfrak{G}$ & $H$ \\
\hline $\mathrm{SL}_{5}$ & $5: 4$ \\
$\mathrm{SL}_{6}$ & $\mathrm{PGL}_{2}(5)$ \\
$\mathrm{SL}_{9}$ & $\mathrm{~L}_{2}(8), \mathrm{L}_{2}(8): 3$ \\
\hline $\mathrm{Spin}_{7}$ & $7: 3,7: 6, \mathrm{~L}_{3}(2)$ \\
$\mathrm{Spin}_{8}$ & $2^{3}: 7,2^{3}: 7: 3, \mathrm{~L}_{2}(7), \mathrm{PGL}_{2}(7), 2^{3}: \mathrm{L}_{3}(2)$ \\
$\mathrm{Spin}_{9}$ & $\mathrm{~L}_{2}(8), \mathrm{L}_{2}(8): 3$ \\
$\operatorname{Spin}_{10}$ & $\mathrm{PGL}_{2}(9), M_{10}, \operatorname{Aut}\left(\mathfrak{A}_{6}\right)$ \\
$\operatorname{Spin}_{11}$ & $M_{11}$ \\
$\operatorname{Spin}_{12}$ & $M_{12}$ \\
\hline
\end{tabular}

We now turn to the almost quasi-simple case. For this we first need the following lemma: 
Lemma 7.19. Let $n \geq 13$ be an integer. Then the interval $\left(\frac{2}{3} n, n\right]$ contains at least two different primes.

Proof. The statement can be checked directly if $13 \leq n \leq 37$. Let $n \geq 38$. Then $x=2 n / 3>25$, hence the intervals $(x, 6 x / 5)$ and $(6 x / 5,36 x / 25)$ both contain at least one prime, cf. [22], whence the result follows.

For a finite group $G$, let $d(G)$ denote the smallest degree of a faithful complex projective representation of $G$.

Lemma 7.20. Let $S$ be a finite simple group with $d(S) \geq 13$. Suppose that

(i) $S$ has a faithful projective complex representation $\Phi$ of degree e, and

(ii) $|S|$ has at least two prime divisors $p, p^{\prime}$ with $p>p^{\prime}>\frac{2}{3} e$.

Then either $S=\mathfrak{A}_{n}$ and $\Phi$ is the smallest representation of degree $n-1$ of $S$, or $S=\mathrm{L}_{2}(q)$ with $q=p, p^{\prime}=(q \pm 1) / 2$ and $e=(q \pm 1) / 2$.

Proof. First suppose that $S=\mathfrak{A}_{n}$. Then $e \geq d(S) \geq 13$, whence $n \geq 14$. Next, $n \geq p>2 e / 3$, whence $e<3 n / 2$. This implies by [26] and [21] that $e=n-1$ and $\Phi$ is the smallest complex representation of degree $n-1$.

Next, direct check using [1] shows that $S$ cannot be a sporadic simple group. So we may assume that $S$ is of Lie type. Clearly, $p>2 e / 3 \geq 2 d(S) / 3$, whence $13 \leq d(S)<3 p / 2$. This condition excludes all finite groups of Lie type, except possibly $S=\mathrm{S}_{2 n}(q)$ with $n \geq 2$ and $q$ odd, $\mathrm{L}_{n}(q)$ with $n \geq 2$, $\mathrm{U}_{n}(q)$ with $n \geq 3$. Assume $S=\mathrm{S}_{2 n}(q), n \geq 2$, and $q$ odd. Since $d(S) \geq 13$, we have $q^{n} \geq 27$ and $d(S)=\left(q^{n}-1\right) / 2$. Now

$$
p>2 d(S) / 3=\left(q^{n}-1\right) / 3>\max \left\{\left(q^{n-1}+1\right) / 2,\left(q^{n}+1\right) / 4, q, 2\right\} .
$$

But $p$ is a prime divisor of $|S|$, hence $p=\left(q^{n} \pm 1\right) / 2$. The same holds for $p^{\prime}$. Thus both $\left(q^{n}-1\right) / 2$ and $\left(q^{n}+1\right) / 2$ are primes, a contradiction. The cases $\mathrm{L}_{n}(q), n \geq 3$, and $\mathrm{U}_{n}(q)$ can be excluded similarly.

Proposition 7.21. Let $S$ be a finite simple group with $d(S) \geq 13$ and $G$ a finite group such that $S \leq G / Z(G) \leq \operatorname{Aut}(S)$. Suppose that $G$ can be embedded in a simple simply-connected complex classical group $\mathfrak{G}$ in such a way that $G$ acts irreducibly on all fundamental representations of $\mathfrak{G}$. Then $S=\mathfrak{A}_{n}, Z(G) \times \mathfrak{A}_{n} \leq G \leq Z(G) * \mathfrak{S}_{n}, \mathfrak{G}=\mathrm{SL}_{n-1}(\mathbb{C})$.

Proof. Let $K=G^{(\infty)}, C=C_{G}(K)=Z(G), L=K * C$. Then $K$ is a finite quasi-simple group and $K / Z(K) \simeq S$. Let $V$ be the natural module for $\mathfrak{G}$ and let $d=\operatorname{dim}(V)$. By Clifford's Theorem, $\left.V\right|_{K}$ is a direct sum of irreducible $K$-modules of dimension say $e$, each of which is a faithful projective representation of $S$. In particular, $d \geq e \geq d(S) \geq 13$. By Lemma 7.19, one can find primes $p>p^{\prime}$ in the interval $\left(\frac{2}{3} d, d\right]$. Since $d \geq 13$, we have $p>p^{\prime} \geq[d / 2]+3$. Now the $(d-p+1)^{\text {th }}$-fundamental representation 
$W$ of $\mathfrak{G}$ has dimension

$$
\left(\begin{array}{c}
d \\
d-p+1
\end{array}\right)=\frac{p(p+1) \ldots d}{(d-p+1) !}
$$

if $\mathfrak{G}$ is of type $A, B$ or $D$. If $\mathfrak{G}$ is of type $C$, then the $(d-p+3)^{\text {th }}$-fundamental representation $W$ of $\mathfrak{G}$ has dimension

$$
\left(\begin{array}{c}
d \\
d-p+3
\end{array}\right)-\left(\begin{array}{c}
d \\
d-p+1
\end{array}\right)=\frac{(p-2)(p-1) p \ldots d}{(d-p+3) !}-\frac{p(p+1) \ldots d}{(d-p+1) !} .
$$

In either case, $\operatorname{dim}(W)$ is divisible by $p$.

By assumption, $\left.W\right|_{G}$ is irreducible. Hence by Clifford's Theorem, $\left.W\right|_{L}$ is a direct sum of $t$ irreducible $L$-modules, each of dimension $l$, and $t \mid(G: L)$. Clearly, $p$ divides $l t$. Assume that $p$ does not divide $|S|$. Since $p>2 d(S) / 3$, we can conclude that $p$ does not divide $|\operatorname{Aut}(S)|$ and $|\operatorname{Mult}(S)|$. Thus $p$ does not divide $(G: L)$, and so $p \mid l$. But $l$ is an irreducible degree of $L=K * C$ and $C=Z(G)$ is abelian. Therefore, $p$ divides $|K|$, a contradiction. Consequently, $p$ divides $|S|$. Similarly, $p^{\prime}$ divides $|S|$. Now we can apply Lemma 7.20. Observe that the case $S=\mathrm{L}_{2}(q)$ with $q=p$ is impossible: $(G: L) \leq 2$ and $d \geq e \geq(q-1) / 2$, and so already the third fundamental representation has degree too big to be a sum of $\leq 2$ irreducible representations of $L$.

The simple groups with a nontrivial projective complex representation of degree at most twelve are $\mathfrak{A}_{5,6,7,8,9,10,11,12,13}, \mathrm{~L}_{2}(7,8,11,13,17,19,23,25)$, $\mathrm{L}_{3}(3,4), \mathrm{U}_{3}(3,4), \mathrm{U}_{4}(2,3), \mathrm{U}_{5}(2), \mathrm{S}_{4}(5), \mathrm{S}_{6}(2), \mathrm{O}_{8}^{+}(2), G_{2}(4), M_{11}, M_{12}, J_{2}$, $M_{22}$ and $S u z$. The character tables of all these groups are contained in the Atlas [1] and can be checked directly.

The quasi-simple subgroups of simple complex exceptional groups have been classified, see for example [4] for references. Apart from the groups listed above, these are $\mathfrak{A}_{14,15,16,17}, \mathrm{~L}_{2}(16,27,29,31,32,37,41,49,61), \mathrm{L}_{3}(5)$, $\mathrm{U}_{3}(8), G_{2}(3),{ }^{3} D_{4}(2),{ }^{2} B_{2}(8)$ and ${ }^{2} F_{4}(2)^{\prime}$. The groups $E_{7}(\mathbb{C})$ and $E_{8}(\mathbb{C})$ both have fundamental representations of degrees divisible by 13 and by 19 . None of the quasi-simple groups from the above lists has this property. The only groups with an irreducible complex representation of degree 27 are $\mathfrak{A}_{9}, \mathrm{~L}_{2}(53), \mathrm{U}_{3}(3), \mathrm{S}_{6}(2), 3 . \mathrm{O}_{7}(3), 3 . G_{2}(3)$, and ${ }^{2} F_{4}(2)^{\prime}$. None of these has a character of degree 2925 . The only groups with an irreducible complex representation of degree 26 are $\mathrm{L}_{2}(25), \mathrm{L}_{2}(27), \mathrm{L}_{2}(53), \mathrm{L}_{4}(3),{ }^{3} D_{4}(2)$ and ${ }^{2} F_{4}(2)^{\prime}$. Of these, only ${ }^{3} D_{4}(2)$ has characters of degree $52,273,1274$ as well. The only groups with an irreducible complex representation of degree 7 are $\mathfrak{A}_{8}, \mathrm{~L}_{2}(8), \mathrm{L}_{2}(13), \mathrm{U}_{3}(3)$ and $\mathrm{S}_{6}(2)$. Of these, only $\mathfrak{A}_{8}, \mathrm{~L}_{2}(13)$ and $\mathrm{U}_{3}(3)$ also have a projective character of degree 14 . But $2 . \mathfrak{A}_{8}$ is known not to be contained in $G_{2}(\mathbb{C})$. 
Table 7.22. Almost quasi-simple subgroups $G$ of complex simple Lie groups $\mathfrak{G}$ irreducible in all fundamental representations.

\begin{tabular}{|l|l|c|c|}
\hline $\mathfrak{G}$ & $G$ & ext. & remarks \\
\hline $\mathrm{SL}_{n}$ & $\mathfrak{S}_{n+1}$ & & $\operatorname{Weyl}\left(A_{n}\right)$ \\
$\mathrm{SL}_{3}$ & $\mathfrak{A}_{5}$ & & $\operatorname{Weyl}\left(H_{3}\right)$ \\
$\mathrm{SL}_{3}$ & $\mathrm{~L}_{3}(2)$ & & $\operatorname{CRG}(24)$ \\
$\mathrm{SL}_{3}$ & $3 . \mathfrak{A}_{6}$ & & $\operatorname{CRG}(27)$ \\
$\mathrm{SL}_{4}$ & $2 . \mathrm{L}_{3}(2)$ & & \\
$\mathrm{SL}_{4}$ & $2 . \mathrm{U}_{4}(2)$ & & $\operatorname{CRG}(32)$ \\
$\mathrm{SL}_{4}$ & $2 . \mathfrak{A}_{7}$ & & \\
$\mathrm{SL}_{5}$ & $\mathrm{~L}_{2}(11)$ & & \\
$\mathrm{SL}_{5}$ & $\mathrm{U}_{4}(2)$ & & $\operatorname{CRG}(33)$ \\
$\mathrm{SL}_{6}$ & $6 . \mathrm{L}_{3}(4) \cdot 2$ & & \\
$\mathrm{SL}_{6}$ & $\mathrm{U}_{4}(2) \cdot 2$ & & $\operatorname{Weyl}\left(E_{6}\right)$ \\
$\mathrm{SL}_{6}$ & $6_{1} \cdot \mathrm{U}_{4}(3)$ & \multirow{2}{*}{$2_{2}$} & $\operatorname{CRG}(34)$ \\
$\mathrm{SL}_{6}$ & $6 . \mathfrak{A}_{7}$ & & \\
$\mathrm{SL}_{7}$ & $\mathrm{~S}_{6}(2)$ & & $\operatorname{Weyl}\left(E_{7}\right)$ \\
$\mathrm{SL}_{8}$ & $4_{1} \cdot \mathrm{L}_{3}(4) .2$ & & \\
$\mathrm{SL}_{8}$ & $2 . \mathrm{O}_{8}^{+}(2) .2$ & & $\operatorname{Weyl}\left(E_{8}\right)$ \\
$\mathrm{SL}_{10}$ & $2 . M_{22} .2$ & & \\
\hline
\end{tabular}

In Table 7.22, the examples in Lie groups of type $A$ or $C$ can be seen to occur (using GAP for instance). Gross observed (cf. for instance [14]) that $\mathrm{U}_{3}(3)$, resp. ${ }^{3} D_{4}(2)$, give rise to examples for type $G_{2}$, resp. $F_{4}$. Next, let $G=\mathrm{L}_{2}(13)$. It is known that $G$ embeds in $\mathfrak{G}=G_{2}(\mathbb{C})$, cf. [4]. If $\omega$ is the fundamental representation of degree 7 of $\mathfrak{G}$, then $\omega^{\prime}:=\Lambda^{2}(\omega)-\omega$ is the fundamental representation of degree 14 of $\mathfrak{G}$. Now it is easy to check that the restrictions of $\omega$ and $\omega^{\prime}$ to $G$ are irreducible.

Finally, let $\mathfrak{G}=\operatorname{Spin}_{n}(\mathbb{C})$. If $n=8$ then clearly $G=2 . \mathrm{O}_{8}^{+}(2)$ or $2 . \mathfrak{A}_{9}$ embeds in $\mathfrak{G}$, and since $d(G)=8$ one sees that each $G$ gives rise to an example in $\mathfrak{G}$. Let $n=7$ and let $\omega_{1}$ be the fundamental representation of $\mathfrak{G}$ (on a 7 -dimensional module $V$ ). Let $G=\mathrm{L}_{2}(8) .3$ or $2 . \mathrm{S}_{6}(2)$. Then $G$ embeds in $\mathrm{O}_{7}(\mathbb{C})=\mathrm{O}(V)$, and the restrictions of $\omega_{1}$ and $\omega_{2}=\Lambda^{2}\left(\omega_{1}\right)$ to $G$ are irreducible. It is known that the square of the third fundamental 
Table 7.22. Almost quasi-simple subgroups $G$ of complex simple Lie groups $\mathfrak{G}$ irreducible in all fundamental representations (continued).

\begin{tabular}{|l|l|c|}
\hline $\mathfrak{G}$ & $G$ & ext. \\
\hline $\mathrm{Sp}_{4}$ & $2 . \mathfrak{A}_{5}$ & \\
$\mathrm{Sp}_{4}$ & $2 . \mathfrak{A}_{6}$ & $2_{1}$ \\
$\mathrm{Sp}_{6}$ & $2 . \mathrm{L}_{2}(13)$ & \\
$\mathrm{Sp}_{6}$ & $2 . J_{2}$ & \\
\hline $\mathrm{Spin}_{7}$ & $\mathrm{~L}_{2}(8) .3$ & \\
$\mathrm{Spin}_{7}$ & $2 . \mathrm{S}_{6}(2)$ & \\
$\mathrm{Spin}_{8}$ & $2 . \mathrm{O}_{8}^{+}(2)$ & 2 \\
$\mathrm{Spin}_{8}$ & $2 . \mathfrak{A}_{9}$ & \\
\hline$G_{2}$ & $\mathrm{~L}_{2}(13)$ & \\
$G_{2}$ & $\mathrm{U}_{3}(3)$ & 2 \\
$F_{4}$ & ${ }^{3} D_{4}(2)$ & 3 \\
\hline
\end{tabular}

representation, $\omega_{3}$, of $\mathfrak{G}$ is just the representation of $\mathfrak{G}$ on the even part of the Clifford algebra $C(V)$. Hence

$$
\omega_{3}^{2}=1+\Lambda^{2}\left(\omega_{1}\right)+\Lambda^{4}\left(\omega_{1}\right)+\Lambda^{6}\left(\omega_{1}\right)=\sum_{i=0}^{3} \Lambda^{i}\left(\omega_{1}\right) .
$$

In particular, if $g \in G$ is of order 9 , then $\omega_{3}(g)^{2}=1$. Since $\left.\omega_{3}\right|_{G}$ is a complex representation of degree 8 , this implies that $\left.\omega_{3}\right|_{G}$ is irreducible.

Thus all examples in Table 7.22 do indeed occur. This completes the proof of Theorem 1.3.

\section{References}

[1] J.H. Conway, R.T. Curtis, S.P. Norton, R.A. Parker and R.A. Wilson, Atlas of Finite Groups, Clarendon Press, Oxford, 1985, MR 88g:20025, Zbl 568.20001.

[2] M. Geck, G. Hiss, F. Lübeck, G. Malle and G. Pfeiffer, CHEVIE - A system for computing and processing generic character tables, AAECC, 7 (1996), 175-210, MR 99m:20017, Zbl 847.20006.

[3] D. Gluck and K. Magaard, Cross-characteristic character and fixed-point space ratios for groups of Lie type, J. Algebra, 204 (1998), 188-201, MR 99j:20019, Zbl 912.20009.

[4] R.L. Griess and A.J.E. Ryba, Finite simple groups which projectively embed in an exceptional Lie group are classified!, Bull. Amer. Math. Soc., 36 (1999), 75-93, MR 99g:20085, Zbl 916.22008. 
[5] R.M. Guralnick, K. Magaard, J. Saxl and P.H. Tiep, Cross characteristic representations of odd characteristic symplectic groups and unitary groups, submitted, 2000.

[6] R.M. Guralnick, T. Pentilla, C. Praeger and J. Saxl, Linear groups with orders having certain primitive prime divisors, Proc. London Math. Soc., 78 (1999), 167-214, MR 99m:20113.

[7] G. Hiss and G. Malle, Low-dimensional representations of special unitary groups, J. Algebra, 236 (2001), 745-767, CMP 1813499.

[8] G. Hiss and J. Shamash, 3-blocks and 3-modular characters of $G_{2}(q)$, J. Algebra, 131 (1990), 371-387, MR 91h:20017, Zbl 711.20008.

[9] C. Hoffman, Cross characteristic projective representations for some classical groups, J. Algebra, 229 (2000), 666-677, MR 2001f:20029, Zbl 962.20034.

[10] L_ Low dimensional projective representations for some exceptional finite groups of Lie type, preprint, 1997.

[11] J. Humphreys, Introduction to Lie Algebras and Representation Theory, Springer, New York, 1972, MR 48 \#2197, Zbl 254.17004.

[12] C. Jansen, K. Lux, R. Parker and R. Wilson, An Atlas of Brauer Characters, Clarendon Press, Oxford, 1995, MR 96k:20016, Zbl 831.20001.

[13] V. Landázuri and G.M. Seitz, On the minimal degrees of the projective representations of the finite Chevalley groups, J. Algebra, 32 (1974), 418-443, MR 50 \#13299, Zbl 325.20008.

[14] W. Lempken, B. Schröder and P.H. Tiep, Symmetric squares and lattice minima, J. Algebra, 240(1) (2001), 185-208, CMP 1830550.

[15] M.W. Liebeck and G.M. Seitz, On the subgroup structure of classical groups, Invent. Math., 134 (1998), 427-453, MR 99h:20074, Zbl 920.20039.

[16] D. Livingstone and A. Wagner, Transitivity of finite permutation groups on unordered sets, Math. Z., 90 (1965), 339-403, MR 32 \#4183, Zbl 136.28101.

[17] F. Lübeck, Smallest degrees of representations of exceptional groups of Lie type, Comm. in Algebra, 29 (2001), 2147-2169.

[18] K. Magaard and G. Malle, Irreducibility of alternating and symmetric squares, Manuscripta Math., 95 (1998), 169-180, MR 99a:20011, Zbl 919.20009.

[19] K. Magaard and P.H. Tiep, Irreducible tensor products of representations of finite quasi-simple groups of Lie type, Modular Representation Theory of Finite Groups, M.J. Collins, B.J. Parshall, L.L. Scott eds., de Gruyter, 2001, 239-262.

[20] G. Malle, Almost irreducible tensor squares, Comm. Algebra, 27 (1999), 1033-1051, MR 99m:20007, Zbl 931.20009.

[21] R. Rasala, On the minimal degrees of characters of $S_{n}$, J. Algebra, 45 (1977), 132-181, MR 55 \#477, Zbl 348.20009.

[22] P. Ribenboim, The Book of Prime Number Records, Springer, New York, 1988, MR 89e:11052, Zbl 642.10001.

[23] G.M. Seitz and A.E. Zalesskii, On the minimal degrees of projective representations of the finite Chevalley groups, II, J. Algebra, 158 (1993), 233-243, MR 94h:20017, Zbl 789.20014.

[24] P.H. Tiep, Dual pairs and low-dimensional representations of finite classical groups, in preparation. 
[25] P.H. Tiep and A.E. Zalesskii, Minimal characters of the finite classical groups, Comm. Algebra, 24 (1996), 2093-2167, MR 97f:20018, Zbl 901.20031.

[26] A. Wagner, An observation on the degrees of projective representations of the symmetric and alternating groups over an arbitrary field, Arch. Math., 29 (1977), 583-589, MR 57 \#444, Zbl 383.20009.

[27] A.E. Zalesskii, Eigenvalues of matrices of complex representations of finite groups of Lie type, Lecture Note Math., 1352, Springer, New York, (1988), 206-218, MR 90d:20084, Zbl 661.20032.

[28] K. Zsigmondy, Zur theorie der potenzreste, Monatsh. Math. Phys., 3 (1892), 265-284.

Received February 2, 2000 and revised November 6, 2000. This paper was written while the authors were participating in the special program on Galois groups and fundamental groups at the MSRI (Berkeley). It is a pleasure to thank the institute for its hospitality. The first author was supported by the NSA and a career development chair from Wayne State University. The third author was supported in part by a research award from the College of Liberal Arts and Sciences, University of Florida.

Department of Mathematics

WAYNe State University

DETROIT, MI 48202

E-mail address: kaym@math.wayne.edu

FACHBEREICH Mathematik/Informatik

UNIVERSITÄT KASSEL

HEINRICH-PletT-STRASSE 40

D-34132 Kassel, Germany

E-mail address: malle@mathematik.uni-kassel.de

Department of Mathematics

UNIVERSITY OF FLORIDA

Gainesville, FL 32611

E-mail address: tiep@math.ufl.edu 